\title{
THREE EMBEDDINGS OF THE KLEIN SIMPLE GROUP INTO THE CREMONA GROUP OF RANK THREE
}

\author{
IVAN CHELTSOV AND CONSTANTIN SHRAMOV
}

\begin{abstract}
We study the action of the Klein simple group $\operatorname{PSL}_{2}\left(\mathbb{F}_{7}\right)$ consisting of 168 elements on two rational threefolds: the three-dimensional projective space and a smooth Fano threefold $X$ of anticanonical degree 22 and index 1 . We show that the Cremona group of rank three has at least three non-conjugate subgroups isomorphic to $\mathrm{PSL}_{2}\left(\mathbb{F}_{7}\right)$. As a by-product, we prove that $X$ admits a KählerEinstein metric, and we construct a smooth polarized $K 3$ surface of degree 22 with an action of the group $\operatorname{PSL}_{2}\left(\mathbb{F}_{7}\right)$.
\end{abstract}

Unless explicitly stated otherwise, varieties are assumed to be projective, normal and complex.

\section{INTRODUCTION}

The Cremona group of rank $n$, usually denoted by $\mathrm{Cr}_{n}(\mathbb{C})$, is the group of birational automorphisms of the complex projective space $\mathbb{P}^{n}$. It is well-known that $\mathrm{Cr}_{1}(\mathbb{C})=\operatorname{Aut}\left(\mathbb{P}^{1}\right) \cong \mathrm{PGL}_{2}(\mathbb{C})$. For $n \geqslant 2$, the structure of the group $\mathrm{Cr}_{n}(\mathbb{C})$ is much more complicated than of its subgroup $\operatorname{Aut}\left(\mathbb{P}^{n}\right)$. So one possible way to study the Cremona groups of high rank is by analyzing their finite subgroups.

Finite subgroups in $\mathrm{Cr}_{1}(\mathbb{C}) \cong \mathrm{PGL}_{2}(\mathbb{C})$ are cyclic, $\mathbb{Z}_{2} \times \mathbb{Z}_{2}$, dihedral, $\mathrm{A}_{4}$, and $\mathrm{A}_{5}$. Two finite subgroups in $\mathrm{Cr}_{1}(\mathbb{C})$ are conjugate if and only if they are isomorphic. Finite subgroups in $\mathrm{Cr}_{2}(\mathbb{C})$ have been almost completely classified in [12. This is an important and still active research direction originating in the works of Kantor, Bertini, and Wiman.

Example 1.1 ([5, Theorem B.2]). Let $G$ be a finite simple non-abelian subgroup. Then $\mathrm{Cr}_{2}(\mathbb{C})$ has a subgroup isomorphic to $G$ if and only if $G$ is one of the following groups: $\mathrm{A}_{5}, \mathrm{PSL}_{2}\left(\mathbb{F}_{7}\right)$ or $\mathrm{A}_{6}$. The group $\mathrm{Cr}_{2}(\mathbb{C})$ has exactly 3,2 and 1 non-conjugate subgroups isomorphic to $\mathrm{A}_{5}, \mathrm{PSL}_{2}\left(\mathbb{F}_{7}\right)$ and $\mathrm{A}_{6}$, respectively.

Much less is known about finite subgroups in $\mathrm{Cr}_{3}(\mathbb{C})$. In fact, they were so poorly understood until recently that Serre asked the following

Question 1.2 ([38, Question 6.0]). Does there exist a finite group which is not embeddable in $\mathrm{Cr}_{3}(\mathbb{C})$ ?

Inspired by Question 1.2 and using methods of modern three-dimensional birational geometry, Prokhorov proved the following

Theorem 1.3 ([35, Theorem 1.3]). Suppose that $G$ is a finite simple non-abelian subgroup. Then $\mathrm{Cr}_{3}(\mathbb{C})$ has a subgroup isomorphic to $G$ if and only if $G$ is one of the following groups: $\mathrm{A}_{5}, \mathrm{PSL}_{2}\left(\mathbb{F}_{7}\right), \mathrm{A}_{6}, \mathrm{~A}_{7}, \mathrm{PSL}_{2}\left(\mathbb{F}_{8}\right)$ or $\mathrm{PSU}_{4}\left(\mathbb{F}_{2}\right)$.

The authors thank I. Dolgachev, Th. Köppe, A. Kuznetsov and Yu. Prokhorov for their help. The first author thanks the Max-Planck-Institute für Mathematik at Bonn for hospitality.

The authors were partially supported by AG Laboratory GU-HSE, RF government grant 11 11.G34.31.0023. The second author was partially supported by the grants RFFI 08-01-00395-a, RFFI 11-01-00185-a, RFFI 11-01-00336-a and N.Sh.-4713.2010.1. 
The technique introduced in [35] allows one to handle finite subgroups in $\mathrm{Cr}_{3}(\mathbb{C})$ in a similar way to ones in $\mathrm{Cr}_{2}(\mathbb{C})$. Moreover, the proof of Theorem 1.3 gives us much more than just a classification. For instance, one can easily use this proof to obtain the following

Theorem 1.4 ([8, Corollary 1.23], [1]). Up to conjugation the group $\mathrm{Cr}_{3}(\mathbb{C})$ contains exactly one subgroup isomorphic to $\mathrm{PSL}_{2}\left(\mathbb{F}_{8}\right)$, exactly one subgroup isomorphic to $\mathrm{A}_{7}$ and exactly two subgroups isomorphic to $\mathrm{PSU}_{4}\left(\mathbb{F}_{2}\right)$.

Unfortunately, the proof of Theorem 1.3 can not be applied to study the conjugacy classes in $\mathrm{Cr}_{3}(\mathbb{C})$ of the subgroups $\mathrm{A}_{5}, \mathrm{PSL}_{2}\left(\mathbb{F}_{7}\right)$, and $\mathrm{A}_{6}$, mostly because these subgroups are rather small and $\mathrm{Cr}_{3}(\mathbb{C})$ contains many non-conjugate embeddings of these groups. For example, nothing is known so far about the number of non-conjugate subgroups in $\mathrm{Cr}_{3}(\mathbb{C})$ that are isomorphic to the group $\mathrm{A}_{5}$. As for the group $\mathrm{A}_{6}$, we have the following

Theorem 1.5 $([\mathbb{8}])$. The group $\mathrm{Cr}_{3}(\mathbb{C})$ has at least 5 non-conjugate subgroups isomorphic to $\mathrm{A}_{6}$.

The main purpose of this paper is to prove the following

Theorem 1.6. The group $\mathrm{Cr}_{3}(\mathbb{C})$ has at least 3 non-conjugate subgroups isomorphic to $\mathrm{PSL}_{2}\left(\mathbb{F}_{7}\right)$.

From now on we denote the group $\mathrm{PSL}_{2}\left(\mathbb{F}_{7}\right)$ by $G$. Any embedding $G \hookrightarrow \mathrm{Cr}_{3}(\mathbb{C})$ arises from some rational threefold $X$ admitting a faithful action of the group $G$ (for details see [12], [35]), and the first examples of such embeddings come from representation theory. Moreover, the normalizer of the groups $G$ in $\mathrm{Cr}_{3}(\mathbb{C})$ is isomorphic to the normalizer of the group $G$ in $\operatorname{Bir}(X)$. The latter group coincides with the group of $G$-equivariant birational self-maps of $X$, which we denote by $\operatorname{Bir}^{G}(X)$. Note that the group of $G$-equivariant biregular self-maps of $X$ coincides with the normalizer of $G$ in $\operatorname{Aut}(X)$, which we denote by $\operatorname{Aut}^{G}(X)$.

Example 1.7. Up to conjugation, the group $\operatorname{Aut}\left(\mathbb{P}^{3}\right)$ has two subgroups isomorphic to $\operatorname{PSL}_{2}\left(\mathbb{F}_{7}\right)$. The first subgroup (we will call it a subgroup of type $(\mathbf{I})$ ) arises from a faithful reducible four-dimensional representation of the group $\mathrm{PSL}_{2}\left(\mathbb{F}_{7}\right)$, which splits as a sum of an irreducible three-dimensional representation and a trivial one. The second subgroup (we will call it a subgroup of type (II)) arises from a faithful irreducible four-dimensional representation of the group $\mathrm{SL}_{2}\left(\mathbb{F}_{7}\right)$ (see [9]). Note that the subgroup of type (I) fixes a point in $\mathbb{P}^{3}$, while the subgroup of type (II) does not fix any point in $\mathbb{P}^{3}$.

The next example of an embedding $G \hookrightarrow \mathrm{Cr}_{3}(\mathbb{C})$ comes from the celebrated Klein quartic curve - the unique genus 3 curve with an action of the group $\mathrm{PSL}_{2}\left(\mathbb{F}_{7}\right)$ (see [24]).

Example $1.8\left([26],\left[18,[28,[27])\right.\right.$. Let $\mathfrak{C}$ be the quartic curve in $\mathbb{P}^{2}$ that is given by

$$
x y^{3}+y z^{3}+z x^{3}=0 \subset \mathbb{P}^{2} \cong \operatorname{Proj}(\mathbb{C}[x, y, z]),
$$

put $X=\operatorname{VSP}(\mathfrak{C}, 6)$ (see Definition $\underline{\mathrm{A} .3}$ ). Then $X$ is a rational smooth Fano threefold such that

$$
\operatorname{Aut}(X) \cong \operatorname{Aut}(C) \cong \mathrm{PSL}_{2}\left(\mathbb{F}_{7}\right),
$$

the group $\operatorname{Pic}(X)$ is generated by $-K_{X}$, and $-K_{X}^{3}=22$ (see Section 4 , Appendix $\mathrm{A}$ and [19]). One can show that $X$ is a compactification of the moduli space of $(1,7)$ polarized abelian surfaces (see [26], [18] and [28] for details). 
In Sections 5 and 6, we prove the following

Theorem 1.9. Let $G$ be a subgroup in $\operatorname{Aut}\left(\mathbb{P}^{3}\right)$ such that $G \cong \mathrm{PSL}_{2}\left(\mathbb{F}_{7}\right)$ is of type (II) in the notation of Example 1.7. Then the variety $\mathbb{P}^{3}$ is $G$-birationally rigid (see [8, Definition 1.9]), and there is a $G$-equivariant birational non-biregular involution $\tau \in \mathrm{Cr}_{3}(\mathbb{C})$ such that

$$
\operatorname{Bir}^{G}\left(\mathbb{P}^{3}\right)=\langle G, \tau\rangle \cong \operatorname{PSL}_{2}\left(\mathbb{F}_{7}\right) \times \mathbb{Z}_{2}
$$

Theorem 1.10. Let $X$ be the threefold constructed in Example 1.8. Moreover, $\operatorname{Bir}^{G}(X)=\operatorname{Aut}^{G}(X)=G \cong \mathrm{PSL}_{2}\left(\mathbb{F}_{7}\right)$ and the threefold $X$ is $G$-birationally superrigid (see [8, Definition 1.10]).

It should be pointed out that Theorems 1.10 and 1.9 imply Theorem 1.6. Moreover, Theorem 1.10 also implies the following

Corollary 1.11. Let $G$ and $G^{\prime}$ be subgroups in $\operatorname{Aut}\left(\mathbb{P}^{3}\right)$ such that $G \cong G^{\prime} \cong$ $\mathrm{PSL}_{2}\left(\mathbb{F}_{7}\right)$. Then $G$ and $G^{\prime}$ are conjugate in $\mathrm{Cr}_{3}(\mathbb{C})$ if and only if $G$ and $G^{\prime}$ are conjugate in $\operatorname{Aut}\left(\mathbb{P}^{3}\right)$.

As a by-product of the proof of Theorem 1.10, in Section 6 we prove the following

Theorem 1.12. Let $X$ be a threefold constructed in Example 1.8, and let $R$ be an effective $G$-invariant $\mathbb{Q}$-divisor such that $R \sim_{\mathbb{Q}}-K_{X}$. Then the pair $(X, R)$ is log canonical.

Applying [40, Theorem 2.1], 66, Theorem A.3] and Theorem 1.12, we immediately obtain the following

Corollary 1.13. Let $X$ be a threefold constructed in Example 1.8, Then $X$ has a $G$-invariant Kähler-Einstein metric.

Note that the threefold constructed in Example 1.8 admits both Kähler-Einstein and non-Kähler-Einstein smooth deformations (see [41, Corollary 1.3] and [14, $\S 5.3]$ ). However, there was only one previously known explicit example of a KählerEinstein threefold in this deformation family, which is the famous Mukai-Umemura threefold (see [14, Theorem 5.4.3]).

Remark 1.14. Let $\hat{G}$ be a subgroup in $\mathrm{SL}_{4}(\mathbb{C})$ such that $\hat{G} \cong \mathrm{SL}_{2}\left(\mathbb{F}_{7}\right)$ is of type (II) in the notation of Example 1.7. Then the quotient singularity $\mathbb{C}^{4} / \hat{G}$ is weaklyexceptional (see [33, Definition 4.1]) by [7, Theorem 3.16] and [7, Theorem 4.3], which implies that an assertion similar to Theorem 1.12 holds for $\mathbb{P}^{3}$. Namely, let $G$ be a subgroup in $\operatorname{Aut}\left(\mathbb{P}^{3}\right)$ such that $G \cong \mathrm{PSL}_{2}\left(\mathbb{F}_{7}\right)$ is of type (II) in the notation of Example 1.7, and let $R$ be an effective $G$-invariant $\mathbb{Q}$-divisor on $\mathbb{P}^{3}$ such that $R \sim_{\mathbb{Q}}-K_{\mathbb{P}^{3}}$. Then the pair $\left(\mathbb{P}^{3}, R\right)$ is $\log$ canonical by [7, Theorem 3.16]. In particular, it follows from [4, Theorem 6.4] that one can apply Theorems 1.10 and 1.9 to construct non-conjugate embeddings $G^{n} \hookrightarrow \mathrm{Cr}_{3 n}(\mathbb{C})$ for $n \geqslant 2$.

As an another by-product of the proof of Theorem 1.10, we give an example of a smooth $K 3$ surface admitting a faithful action of the group $\mathrm{PSL}_{2}\left(\mathbb{F}_{7}\right)$ (see Lemma 4.10). This might be a new example (at least we were not able to find it in the literature).

Remark 1.15. There are other known examples of smooth $K 3$ surfaces that admit faithful actions of the group $\operatorname{PSL}_{2}\left(\mathbb{F}_{7}\right)$. The Edge quartic surface in $\mathbb{P}^{3}$ (see [15]), the cyclic cover of degree 4 of the plane branched along the Klein quartic curve $\mathfrak{C}$ (see [29]), the double cover of the plane branched over the Hessian $H$ of the curve $\mathfrak{C}$ 
(see [29]), and the variety of sum of powers $\operatorname{VSP}(10, H)$ (see [36]). We do not know whether or not the surface provided by Lemma 4.10 is isomorphic as a non-polarized smooth $K 3$ surface to one these smooth $K 3$ surfaces.

Let us sketch the proof of Theorem 1.9 (the proof of Theorem 1.10 is similar and simpler). Let $G$ be a subgroup in $\operatorname{Aut}\left(\mathbb{P}^{3}\right)$ such that $G \cong \mathrm{PSL}_{2}\left(\mathbb{F}_{7}\right)$ is of type $(\mathbf{I I})$ in the notation of Example 1.7. Then $\mathbb{P}^{3}$ is not $G$-birationally superrigid, since there are non-biregular $G$-equivariant birational selfmaps of $\mathbb{P}^{3}$. Indeed, it is well-known that there exists a unique smooth $G$-invariant curve $C_{6} \subset \mathbb{P}^{3}$ of degree 6 and genus 3 (see [15]). Blowing up the curve $C_{6}$, and contracting the proper transform of the surface in $\mathbb{P}^{3}$ spanned by three-secants of the curve $C_{6}$, we obtain a non-biregular $G$-equivariant birational involution $\tau: \mathbb{P}^{3} \rightarrow \mathbb{P}^{3}$ which is not defined along the curve $C_{6}$ (see Lemma 3.8, [11, Remark 6.8]).

To prove that $\mathbb{P}^{3}$ is $G$-birationally rigid and $\operatorname{Bir}^{G}\left(\mathbb{P}^{3}\right)=\langle G, \tau\rangle$, it is enough to prove the following statement: for every $G$-invariant linear system $\mathcal{M}$ without fixed components on $\mathbb{P}^{3}$, either the log pair $\left(\mathbb{P}^{3}, \lambda \mathcal{M}\right)$ has non-canonical singularities, or the log pair $\left(\mathbb{P}^{3}, \lambda^{\prime} \tau(\mathcal{M})\right)$ has non-canonical singularities, where $\lambda$ and $\lambda^{\prime}$ are positive rational numbers such that $\lambda \mathcal{M} \sim_{\mathbb{Q}} \lambda^{\prime} \tau(\mathcal{M}) \sim_{\mathbb{Q}}-K_{\mathbb{P}^{3}}$. In fact, the latter property is equivalent to $G$-birational rigidity of $\mathbb{P}^{3}$ and $\operatorname{Bir}^{G}\left(\mathbb{P}^{3}\right)=\langle G, \tau\rangle$ (see [5]).

Applying $\tau$, we may assume that either $\operatorname{mult}_{C_{6}}(\mathcal{M}) \leqslant 1 / \lambda$ or mult $_{C_{6}}(\tau(\mathcal{M})) \leqslant 1 / \lambda^{\prime}$ (this is usually called "untwisting of maximal singularities"). Thus, without loss of generality, we may assume that $\operatorname{mult}_{C_{6}}(\mathcal{M}) \leqslant 1 / \lambda$, which simply means that $\left(\mathbb{P}^{3}, \lambda \mathcal{M}\right)$ is canonical in a general point of the curve $C_{6}$. Now we have to prove that $\left(\mathbb{P}^{3}, \lambda \mathcal{M}\right)$ has non-canonical singularities. In 8 , we proposed a new approach to prove assertions of the latter type, which we call the "multiplication by two trick". Namely, we simply observed that the singularities of the log pair $\left(\mathbb{P}^{3}, 2 \lambda \mathcal{M}\right)$ must be worse than log canonical if the singularities of the log pair $\left(\mathbb{P}^{3}, \lambda \mathcal{M}\right)$ are worse than canonical (see Lemma 2.8, cf. [8, Corollary 2.3]). Although the former condition is much weaker than the latter one, we are in a position to apply the machinery of multiplier ideal sheaves to the log pair $\left(\mathbb{P}^{3}, 2 \lambda \mathcal{M}\right)$ if the singularities $\left(\mathbb{P}^{3}, \lambda \mathcal{M}\right)$ are not canonical.

Using the "multiplication by two trick", we proved in [8] that $\mathbb{P}^{3}$ is $\mathrm{A}_{6}$-birationally rigid (recall that there is a unique subgroup in $\operatorname{Aut}\left(\mathbb{P}^{3}\right)$ that is isomorphic to $A_{6}$ up to conjugation). However, in the present case, we meet two new problems. The first problem is that sometimes we are just unable to prove that $\left(\mathbb{P}^{3}, 2 \lambda \mathcal{M}\right)$ is $\log$ canonical in a general point of some subvariety of $\mathbb{P}^{3}$ even though we believe that this is true. For example, we do not know how to prove that $\left(\mathbb{P}^{3}, 2 \lambda \mathcal{M}\right)$ is log canonical in a general point of the curve $C_{6}$ despite the fact that we know that $\left(\mathbb{P}^{3}, \lambda \mathcal{M}\right)$ is canonical in a general point of the curve $C_{6}$. The second problem is worse: even if $\left(\mathbb{P}^{3}, \lambda \mathcal{M}\right)$ is canonical, the log pair $\left(\mathbb{P}^{3}, 2 \lambda \mathcal{M}\right)$ may still be not log canonical. One can easily construct such examples (see Example 3.11). To solve both problems, we introduce a new technique, which we call localization and isolation of log canonical centers. Let us describe this technique.

Suppose that the singularities of the $\log$ pair $\left(\mathbb{P}^{3}, \lambda \mathcal{M}\right)$ are not canonical. Let us seek for a contradiction. Take $\mu<2 \lambda$ such that the $\log$ pair $\left(\mathbb{P}^{3}, \mu \mathcal{M}\right)$ is strictly $\log$ canonical (see Section 2), and pick up a minimal center $S$ of $\log$ canonical singularities of $\left(\mathbb{P}^{3}, \mu \mathcal{M}\right)$ (see [20], [21], [7, Definition 2.8]). The minimality of the center $S$ implies that the $G$-orbit of $S$ is either a finite set, or a disjoint union of irreducible curves. We use Lemma 2.13 to observe that one may assume that every center of log canonical singularities of the log pair $\left(\mathbb{P}^{3}, \mu \mathcal{M}\right)$ is $g(S)$ for some $g \in \bar{G}$. 
Then applying the Nadel-Shokurov vanishing theorem (see Theorem 2.2, [23, Theorem 9.4.8]) we obtain an upper bound on the number of irreducible components of the $G$-orbit of $S$ with some additional information (for example, if $S$ is a point, then the points in its $G$-orbit must impose independent linear conditions on sections in $\left.H^{0}\left(\mathcal{O}_{\mathbb{P}^{3}}(4)\right)\right)$. If $S$ is a curve, then the Kawamata subadjunction theorem (see Theorem 2.10, [21, Theorem 1]) implies that $S$ is smooth, and we can proceed with applying the Nadel-Shokurov vanishing theorem, the Riemann-Roch theorem, the Castelnuovo bound (see Theorem 2.14, [17, Theorem 6.4]), and the Corti inequality (see Theorem 2.5, [10, Theorem 3.1]), to prove that $S=C_{6}$. If $S$ is a point, then analyzing small $G$-orbits in $\mathbb{P}^{3}$, we see that the $G$-orbit of the point $S$ consists of either 8 or 28 points (see Lemmas 3.2 and 5.12 ). Note that there is a unique $G$-orbit in $\mathbb{P}^{3}$ consisting of 8 points, and there are exactly two $G$-orbits in $\mathbb{P}^{3}$ consisting of 28 points (see Lemma 3.2). Thus, all potentially dangerous (for the proof of Theorem 1.9) $G$-invariant subvarieties in $\mathbb{P}^{3}$ are explicitly described. This is localization of log canonical centers.

Let us denote by $\Sigma$ the union of the curve $C_{6}$, the $G$-orbit consisting of eight points, and both $G$-orbits consisting of 28 points. Now we can use brute force to prove that $\left(\mathbb{P}^{3}, \lambda \mathcal{M}\right)$ is canonical along $\Sigma$ keeping in mind that mult $C_{6}(\mathcal{M}) \leqslant 1 / \lambda$. Then we conclude that $\left(\mathbb{P}^{3}, 2 \lambda \mathcal{M}\right)$ is not log canonical outside of the subset $\Sigma$. Thus, there exists $\mu^{\prime}<2 \lambda$ such that $\left(\mathbb{P}^{3}, \mu^{\prime} \mathcal{M}\right)$ is strictly log canonical outside of $\Sigma$. Let $S^{\prime}$ be a minimal center of log canonical singularities of the log pair $\left(\mathbb{P}^{3}, \mu^{\prime} \mathcal{M}\right)$ that is not contained in $\Sigma$. Note that $S^{\prime}$ exists by construction. If $S^{\prime}$ is a point, then we can proceed as before and easily obtain a contradiction with the Nadel-Shokurov vanishing theorem. Thus, we conclude that $S^{\prime}$ is a curve. If $S^{\prime}$ and $\Sigma$ are disjoint, then we also can proceed as before and obtain a contradiction with the Kawamata subadjunction theorem, the Nadel-Shokurov vanishing theorem, the Riemann-Roch theorem, the Castelnuovo bound, the Corti inequality, etc. This is isolation of log canonical centers.

If $S^{\prime}$ is a curve and $S^{\prime} \cap \Sigma \neq \varnothing$, then we have a problem. Indeed, we can not apply the Kawamata subadjunction theorem to $S^{\prime}$, because the log pair $\left(\mathbb{P}^{3}, \mu^{\prime} \mathcal{M}\right)$ may no longer be log canonical in the points of the finite set $S^{\prime} \cap \Sigma$ if $\mu^{\prime}>\mu$. Recall that $\mu$ is a rational number such that $\left(\mathbb{P}^{3}, \mu \mathcal{M}\right)$ is strictly log canonical, i.e. log canonical and not Kawamata log terminal. To solve this new problem, we have to isolate and localize new $\log$ canonical centers again, i.e. to repeat the previous arguments to the union of $\Sigma$ and new potentially dangerous curves in $\mathbb{P}^{3}$. And then there is a chance that we have to repeat this process again and again. So all together this looks messy. And the proof of Theorem 1.9 is messy. To simplify it, we describe all potentially dangerous log canonical centers before the proof, and then we try to localize and isolate them together at once.

We organize this paper in the following way. In Section 2, we recall several well-known preliminary results. In Section 3, we collect results about the action of the group $\mathrm{PSL}_{2}\left(\mathbb{F}_{7}\right)$ on $\mathbb{P}^{3}$. In Section 4 , we collect results about the threefold constructed in Example 1.8. In Section 5, we prove Theorem 1.9 using results obtained in Section 3. In Section 6, we prove Theorems [1.10] and 1.12] using results obtained in Section 4. In Appendix [A, we describe Mukai's construction of Fano threefolds of degree 22. In Appendix B, we collect elementary results about the groups $\mathrm{PSL}_{2}\left(\mathbb{F}_{7}\right)$ and $\mathrm{SL}_{2}\left(\mathbb{F}_{7}\right)$. Throughout the paper we use standard notation for cyclic, dihedral, symmetric and alternating groups. For a group $\Gamma$ we denote by $2 . \Gamma$ a (non-trivial) central extension of $\Gamma$ by the central subgroup $\mathbb{Z}_{2}$. 


\section{Preliminaries}

Throughout the paper we use the standard language of the singularities of pairs (see [22]). By strictly log canonical singularities we mean log canonical singularities that are not Kawamata log terminal.

Let $X$ be a variety that has at most log terminal singularities, and let $B_{X}$ be a formal $\mathbb{Q}$-linear combination of prime divisors and mobile linear systems $B_{X}=$ $\sum_{i=1}^{r} a_{i} B_{i}+\sum_{j=1}^{s} c_{j} \mathcal{M}_{j}$, where $B_{i}$ and $\mathcal{M}_{j}$ are a prime Weil divisor and a linear system on the variety $X$ that has no fixed components, respectively, and $a_{i}$ and $c_{j}$ are non-negative rational numbers. Note that we can consider $B_{X}$ as a Weil divisor. Suppose that $B_{X}$ is a $\mathbb{Q}$-Cartier divisor.

Definition 2.1. We say that $B_{X}$ and $\left(X, B_{X}\right)$ are mobile if $a_{1}=a_{2}=\ldots=a_{r}=0$.

Let $\pi: \bar{X} \rightarrow X$ be a $\log$ resolution for the log pair $\left(X, B_{X}\right)$, let $\bar{B}_{i}$ and $\overline{\mathcal{M}}_{j}$ be the proper transforms of the divisor $B_{i}$ and the linear system $\mathcal{M}_{j}$ on the variety $\bar{X}$, respectively. Then

$$
K_{\bar{X}}+\sum_{i=1}^{r} a_{i} \bar{B}_{i}+\sum_{j=1}^{s} c_{j} \overline{\mathcal{M}}_{j} \sim_{\mathbb{Q}} \pi^{*}\left(K_{X}+B_{X}\right)+\sum_{i=1}^{m} d_{i} E_{i},
$$

where $E_{i}$ is an exceptional divisor of the morphism $\pi$, and $d_{i}$ is a rational number. Put

$$
\mathcal{I}\left(X, B_{X}\right)=\pi_{*}\left(\mathcal{O}_{\bar{X}}\left(\sum_{i=1}^{m}\left\lceil d_{i}\right\rceil E_{i}-\sum_{i=1}^{r}\left\lfloor a_{i}\right\rfloor B_{i}\right)\right),
$$

and recall that $\mathcal{I}\left(X, B_{X}\right)$ is known as the multiplier ideal sheaf (see [23, Section 9.2]).

Theorem 2.2 ([23, Theorem 9.4.8]). Let $H$ be a nef and big $\mathbb{Q}$-divisor on $X$ such that $K_{X}+B_{X}+H \sim \mathbb{Q} D$ for some Cartier divisor $D$ on the variety $X$. Then $H^{i}\left(\mathcal{I}\left(X, B_{X}\right) \otimes D\right)=0$ for every $i \geqslant 1$.

Let $\mathcal{L}\left(X, B_{X}\right)$ be a subscheme given by the ideal sheaf $\mathcal{I}\left(X, B_{X}\right)$. Put $\operatorname{LCS}\left(X, B_{X}\right)=\operatorname{Supp}\left(\mathcal{L}\left(X, B_{X}\right)\right)$.

Remark 2.3. If the log pair $\left(X, B_{X}\right)$ is log canonical, then the subscheme $\mathcal{L}\left(X, B_{X}\right)$ is reduced.

Let $Z$ be an irreducible subvariety of the variety $X$.

Definition 2.4 ([20, Definition 1.3]). The subvariety $Z$ is said to be a center of $\log$ canonical singularities (non-log canonical singularities, respectively) of the log pair $\left(X, B_{X}\right)$ if

- either $a_{i} \geqslant 1\left(a_{i}>1\right.$, respectively) and $Z=B_{i}$ for some $i \in\{1, \ldots, r\}$,

- or $d_{i} \leqslant-1\left(d_{i}<-1\right.$, respectively) and $Z=\pi\left(E_{i}\right)$ for some $i \in\{1, \ldots, m\}$ and some $\pi$.

Let $\mathbb{L} \mathbb{C S}\left(X, B_{X}\right)$ and $\mathbb{N L} \mathbb{C S}\left(X, B_{X}\right)$ be the sets of centers of log canonical and non-log canonical singularities of the $\log$ pair $\left(X, B_{X}\right)$, respectively. Then $\mathbb{N L} \mathbb{C} S\left(X, B_{X}\right) \subseteq \mathbb{L} \mathbb{C} \mathbb{S}\left(X, B_{X}\right)$.

Theorem 2.5 ([10, Theorem 3.1]). Suppose that $\operatorname{dim}(X)=2$, the set $\mathbb{N L} \mathbb{C S}\left(X, B_{X}\right)$ contains a point $P \in X \backslash \operatorname{Sing}(X)$, the boundary $B_{X}$ is mobile and $s=1$. Then

$$
\operatorname{mult}_{P}\left(M_{1} \cdot M_{1}^{\prime}\right)>4 / c_{1}^{2}
$$

where $M_{1}$ and $M_{1}^{\prime}$ are general curves in the linear system $\mathcal{M}_{1}$. 
Let us denote by $\operatorname{NLCS}\left(X, B_{X}\right)$ the proper subset of the variety $X$ that is a union of all centers in $\mathbb{N L} \mathbb{C S}\left(X, B_{X}\right)$.

Definition 2.6 ([6, Definition 2.2]). The subvariety $Z$ is said to be a center of canonical singularities (non-canonical singularities, respectively) of the log pair $\left(X, B_{X}\right)$ if $Z=\pi\left(E_{i}\right)$ and $d_{i} \leqslant 0\left(d_{i}<0\right.$, respectively) for some $i \in\{1, \ldots, m\}$ and some choice of the morphism $\pi$.

Let $\mathbb{C} \mathbb{S}\left(X, B_{X}\right)$ and $\mathbb{N} \mathbb{C}\left(X, B_{X}\right)$ be the sets of centers of canonical and noncanonical singularities of the log pair $\left(X, B_{X}\right)$, respectively. Then $\mathbb{N} \mathbb{C}\left(X, B_{X}\right) \subseteq$ $\mathbb{C} \mathbb{S}\left(X, B_{X}\right)$.

Theorem 2.7 ([10, Corollary 3.4]). Suppose that $\operatorname{dim}(X)=3$, the set $\mathbb{N} \mathbb{C S}\left(X, B_{X}\right)$ contains a point $P \in X \backslash \operatorname{Sing}(X)$, the boundary $B_{X}$ is mobile and $s=1$. Then

$$
\operatorname{mult}_{P}\left(M_{1} \cdot M_{1}^{\prime}\right)>4 / c_{1}^{2}
$$

where $M_{1}$ and $M_{1}^{\prime}$ are general surfaces in the linear system $\mathcal{M}_{1}$.

Let us denote by $\operatorname{NCS}\left(X, B_{X}\right)$ the proper subset of the variety $X$ that is a union of all centers in $\mathbb{N} \mathbb{C}\left(X, B_{X}\right)$.

Lemma 2.8. Suppose that $X$ is smooth at a general point of the subvariety $Z$. Then $\mathbb{C S}\left(X, B_{X}\right) \subseteq \mathbb{L} \mathbb{C} \mathbb{S}\left(X, 2 B_{X}\right)$ and $\mathbb{N} \mathbb{C}\left(X, B_{X}\right) \subseteq \mathbb{N L} \mathbb{C} S\left(X, 2 B_{X}\right)$.

Proof. This is obvious, because $X$ is smooth at a general point of $Z$.

Suppose that $Z \in \mathbb{L} \mathbb{C} S\left(X, B_{X}\right)$ and $\left(X, B_{X}\right)$ is log canonical along $Z$.

Lemma 2.9 ([20, Proposition 1.5]). Let $Z^{\prime}$ be a center in $\mathbb{L} \mathbb{C S}\left(X, B_{X}\right)$ such that $Z^{\prime} \neq Z$. Then any irreducible component of the intersection $Z \cap Z^{\prime}$ is an element in $\mathbb{L} \mathbb{C} \mathbb{S}\left(X, B_{X}\right)$.

Suppose that $Z$ is a minimal center in $\mathbb{L} \mathbb{C} S\left(X, B_{X}\right)$ (see [20], 21], [7, Definition 2.8]).

Theorem 2.10 ([21, Theorem 1]). The variety $Z$ is normal and has at most rational singularities. If $\Delta$ is an ample $\mathbb{Q}$-Cartier $\mathbb{Q}$-divisor on $X$, then there exists an effective $\mathbb{Q}$-divisor $B_{Z}$ on the variety $Z$ such that

$$
\left.\left(K_{X}+B_{X}+\Delta\right)\right|_{Z} \sim_{\mathbb{Q}} K_{Z}+B_{Z},
$$

and $\left(Z, B_{Z}\right)$ has Kawamata log terminal singularities.

Let $G$ be a finite subgroup of the group $\operatorname{Aut}(X)$.

Lemma 2.11. Let $P, C$ and $S$ be a point, curve and surface in $X$, respectively. Suppose that $\operatorname{Sing}(X) \not \ngtr P \in C \subset S$ and $\operatorname{dim}(X)=3$. Suppose that $P$ and $C$ are $G$-invariant, and either $G \cong \mathrm{A}_{4}$ or $G \cong \mathbb{Z}_{7} \rtimes \mathbb{Z}_{3}$. Then $\operatorname{mult}_{P}(C) \geqslant 3$ and the surface $S$ is singular at the point $P \in X$.

Proof. Let $\gamma: U \rightarrow X$ be a blow up of the threefold $X$ at the point $P$, let $E$ be the $\gamma$-exceptional divisor, and let $\bar{C}$ be the proper transforms of the curve $C$ on the threefold $U$. Then $\operatorname{mult}_{P}(C) \geqslant|\bar{C} \cap E|$.

The group $G$ naturally acts on $E \cong \mathbb{P}^{2}$. This action comes from a faithfull three-dimensional representation of the group $G$, which must be irreducible, because the group $G$ does not have two-dimensional irreducible representations and the group $G$ is not abelian. Thus $|\bar{C} \cap E| \geqslant 3$, and the points of the set $\bar{C} \cap E$ are not contained in a single line in $E \cong \mathbb{P}^{2}$, which immediately implies that the surface $S$ must be singular at the point $P \in X$. 
Suppose that $B_{X}$ is $G$-invariant. Recall that $\left(X, B_{X}\right)$ is $\log$ canonical.

Remark 2.12. Let $g$ be an elements in $G$. Then $g(Z) \in \mathbb{L} \mathbb{C S}\left(X, B_{X}\right)$. By Lemma 2.9, we have $Z \cap g(Z) \neq \varnothing \Longleftrightarrow Z=g(Z)$.

Suppose that $B_{X}$ is ample. Take an arbitrary rational number $\epsilon>1$.

Lemma 2.13. There is a $G$-invariant linear system $\mathcal{B}$ on the variety $X$ that has no fixed components, and there are rational numbers $\epsilon_{1}$ and $\epsilon_{2}$ such that $1 \geqslant \epsilon_{1} \gg 0$ and $1 \gg \epsilon_{2} \geqslant 0$ and

$$
\mathbb{L} \mathbb{C} S\left(X, \epsilon_{1} B_{X}+\epsilon_{2} \mathcal{B}\right)=\left(\bigsqcup_{g \in G}\{g(Z)\}\right) \bigsqcup \mathbb{N L} \mathbb{C} \mathbb{S}\left(X, \epsilon_{1} B_{X}+\epsilon_{2} \mathcal{B}\right),
$$

the log pair $\left(X, \epsilon_{1} B_{X}+\epsilon_{2} \mathcal{B}\right)$ is log canonical along $g(Z)$ for every $g \in G$, the equivalence $\epsilon_{1} B_{X}+\epsilon_{2} \mathcal{B} \sim_{\mathbb{Q}} \epsilon B_{X}$ holds, and $\mathbb{N L} \mathbb{C} \mathbb{S}\left(X, \epsilon_{1} B_{X}+\epsilon_{2} \mathcal{B}\right)=\mathbb{N L} \mathbb{C S}\left(X, B_{X}\right)$.

Proof. See the proofs of [20, Theorem 1.10] and [21, Theorem 1].

Let $\mathcal{C}$ be a smooth irreducible curve in $\mathbb{P}^{3}$ of genus $g$ and degree $d$.

Theorem 2.14 ([17, Theorem 6.4]). If $\mathcal{C}$ is not contained in a hyperplane in $\mathbb{P}^{3}$, then

$$
g \leqslant\left\{\begin{array}{l}
\frac{(d-2)^{2}}{4} \text { if } d \text { is even, } \\
\frac{(d-1)(d-3)}{4} \text { if } d \text { is odd. }
\end{array}\right.
$$

Suppose, in addition, that $G \cong \mathrm{PSL}_{2}\left(\mathbb{F}_{7}\right)$ and $\mathcal{C}$ admits a faithful action of the group $G$.

Lemma 2.15. Let $\Sigma$ be a $G$-orbit of a point in $\mathcal{C}$. Then $|\Sigma| \in\{24,42,56,84,168\}$.

Proof. This follows from Lemma B.1, since stabilizer subgroups of all points in $X$ are cyclic.

Lemma 2.16. Suppose that $g \leqslant 30$. Then $g \in\{3,8,10,15,17,19,22,24,29\}$, and the number of $G$-orbits in $\mathcal{C}$ consisting of $24,42,56,84$ points can be described as follows:

\begin{tabular}{|c|c|c|c|c|}
\hline genus $g$ & 24 points & 42 points & 56 points & 84 points \\
\hline 3 & 1 & 0 & 1 & 1 \\
\hline 8 & 0 & 1 & 2 & 0 \\
\hline 10 & 1 & 1 & 0 & 1 \\
\hline 15 & 0 & 2 & 1 & 0 \\
\hline 15 & 0 & 0 & 1 & 3 \\
\hline 17 & 1 & 0 & 2 & 0 \\
\hline 19 & 2 & 0 & 0 & 1 \\
\hline 22 & 0 & 3 & 0 & 0 \\
\hline 22 & 0 & 1 & 0 & 3 \\
\hline 24 & 1 & 1 & 1 & 0 \\
\hline 29 & 0 & 0 & 2 & 2 \\
\hline
\end{tabular}

Proof. It follows from the classification of finite subgroups of the group $\mathrm{PGL}_{2}(\mathbb{C})$ that $g \neq 0$, and it follows from the non-solvability of the group $G$ that $g \neq 1$.

Let $\Gamma \subset G$ be a stabilizer of a point in $\mathcal{C}$. Then $\Gamma \cong \mathbb{Z}_{k}$ for $k \in\{1,2,3,4,7\}$ by Lemma B.1. 
Put $\overline{\mathcal{C}}=\mathcal{C} / G$. Then $\overline{\mathcal{C}}$ is a smooth curve of genus $\bar{g}$. The Riemann-Hurwitz formula gives

$$
2 g-2=168(2 \bar{g}-2)+84 a_{2}+112 a_{3}+126 a_{4}+144 a_{7},
$$

where $a_{k}$ is the number of $G$-orbits in $\mathcal{C}$ with a stabilizer of a point isomorphic to $\mathbb{Z}_{k}$.

Since $a_{k} \geqslant 0$, one has $\bar{g}=0$, and $2 g-2=-336+84 a_{2}+112 a_{3}+126 a_{4}+144 a_{7}$, which easily implies the required assertions.

Remark 2.17. We do not claim that every case listed in Lemma 2.16 is realized.

Let $L$ be a $G$-invariant line bundle on the curve $\mathcal{C}$ (see [11, §1]).

Lemma 2.18. If $\operatorname{deg}(L) \leqslant 23$ and $L$ is a $G$-linearizable line bundle (see [11, §1]), then $h^{0}\left(\mathcal{O}_{\mathcal{C}}(L)\right) \notin\{1,2,4,5\}$.

Proof. If $L$ is $G$-linearized, then there is a natural linear action of the group $G$ on $H^{0}\left(\mathcal{O}_{\mathcal{C}}(L)\right)$, and the required assertion follows from Lemma 2.15 and Appendix B.

Theorem 2.19 ([11, Theorem 2.4],[28, Lemma 2.10]). If $g=3$, then $\mathcal{C} \cong \mathfrak{C}$ and there is $\theta \in \operatorname{Pic}(\mathcal{C})$ such that $2 \theta \sim K_{\mathcal{C}}$ and $\operatorname{Pic}^{G}(\mathcal{C})=\langle\theta\rangle$.

Thus, if $g=3$, then $\operatorname{deg}(L)$ is even by Theorem 2.19 , because we assume that $L$ is $G$-invariant.

Lemma 2.20. Suppose that $g=8$ and $L$ is $G$-linearizable. Then $7 \mid \operatorname{deg}(L)$.

Proof. Suppose that $7 \nmid \operatorname{deg}(L)$. Then there are integers $a$ and $b$ such that $14 a+b \operatorname{deg}(L)=8$. Put $D=a K_{\mathcal{C}}+b L$. Then $\operatorname{deg}(D)=8$ and $D$ is a $G$-linearizable line bundle.

By the Riemann-Roch theorem, the Clifford theorem (see 17, Theorem 5.4]) and Lemma 2.18, we have $h^{0}\left(\mathcal{O}_{\mathcal{C}}(D)\right)=3$. Then $h^{0}\left(\mathcal{O}_{\mathcal{C}}\left(K_{\mathcal{C}}-D\right)\right)=2$, which contradicts Lemma 2.18,

Lemma 2.21. Suppose that $g=10$ and $L$ is $G$-linearizable. Then $3 \mid \operatorname{deg}(L)$.

Proof. It follows from [11, page 6] that there exists $G$-linearizable line bundle $\gamma$ on $\mathcal{C}$ of degree 6 that generates the group of all $G$-linearizable line bundles on $\mathcal{C}$. Then it follows from [11, Proposition 2.2] that $3 \mid \operatorname{deg}(L)$.

\section{Projective space}

Let $\zeta$ be a primitive seventh root of unity, let $\hat{G}$ be a subgroup in $\mathrm{SL}_{4}(\mathbb{C})$ such that

$$
\hat{G}=\left\langle\left(\begin{array}{cccc}
1 & 0 & 0 & 0 \\
0 & \zeta & 0 & 0 \\
0 & 0 & \zeta^{4} & 0 \\
0 & 0 & 0 & \zeta^{2}
\end{array}\right), \frac{1}{\sqrt{-1}}\left(\begin{array}{cccc}
1 & 2 & 2 & 2 \\
1 & \zeta+\zeta^{6} & \zeta^{2}+\zeta^{5} & \zeta^{3}+\zeta^{4} \\
1 & \zeta^{2}+\zeta^{5} & \zeta^{3}+\zeta^{4} & \zeta+\zeta^{6} \\
1 & \zeta^{3}+\zeta^{4} & \zeta+\zeta^{6} & \zeta^{2}+\zeta^{5}
\end{array}\right)\right\rangle
$$

and let us denote by the symbol $U_{4}$ the corresponding faithful four-dimensional representation of the group $\hat{G}$ (cf. Appendix $\mathbb{B}$ ). Then $\hat{G} \cong \mathrm{SL}_{2}\left(\mathbb{F}_{7}\right)$ and $U_{4}$ is irreducible (see [25], 9]).

Let $\phi: \mathrm{SL}_{4}(\mathbb{C}) \rightarrow \operatorname{Aut}\left(\mathbb{P}^{3}\right)$ be a natural projection. Put $G=\phi(\hat{G})$. Then $G \cong \mathrm{PSL}_{2}\left(\mathbb{F}_{7}\right)$ is of type $(\mathbf{I I})$ in the notation of Example 1.7

Remark 3.1. It follows from [3, Chapter VII] that $\operatorname{Aut}^{G}\left(\mathbb{P}^{3}\right)=G$. 
Lemma 3.2. Let $P$ be a point in $\mathbb{P}^{3}$, and let $\Sigma$ be its $G$-orbit. Suppose that $|\Sigma| \leqslant 41$. Then either $|\Sigma|=8$ and the orbit $\Sigma$ is unique, or $|\Sigma|=24$ and the orbit $\Sigma$ is unique, or $|\Sigma|=28$ and there are exactly two possibilities for the orbit $\Sigma$.

Proof. It follows from Corollary B.2 that $|\Sigma| \in\{7,8,14,21,24,28\}$, because the representation $U_{4}$ is irreducible (so that $|\Sigma| \neq 1$ ). Let $G_{P}$ be a stabilizer subgroup in $G$ of the point $P$, and let $\hat{G}_{P}$ be the preimage of the subgroup $G_{P}$ under $\phi$. If $|\Sigma|=21$, then $\hat{G}_{P} \cong 2$.D ${ }_{4}$, which is impossible by Lemma B.9. If $|\Sigma| \in\{7,14\}$, then $\hat{G}_{P}$ has a subgroup isomorphic to $2 . \mathrm{A}_{4}$, which is also impossible by Lemma B.9. Thus, we see that $|\Sigma| \in\{8,24,28\}$.

Suppose that $|\Sigma|=8$. Then it follows from Lemmas B.1 and B.7 that $G_{P} \cong \mathbb{Z}_{7} \rtimes \mathbb{Z}_{3}$, the orbit $\Sigma$ does exist, the point $P$ is the unique $G_{P}$-invariant point in $\mathbb{P}^{3}$, and $\Sigma$ is unique, since all subgroups of the group $G$ that are isomorphic to $\mathbb{Z}_{7} \rtimes \mathbb{Z}_{3}$ are conjugate by Lemma B.1.

Suppose that $|\Sigma|=24$. Then $G_{P} \cong \mathbb{Z}_{7}$ and $\hat{G}_{P} \cong \mathbb{Z}_{14}$. Take any $g \in \hat{G}_{P}$ such that $\hat{G}_{P}=\langle g\rangle$, and let $R_{n}$ be a one-dimensional representation of the group $\hat{G}_{P}$ such that $g$ acts on $R_{n}$ by multiplication by $-\zeta^{n}$. For a suitable choice of $g$, we

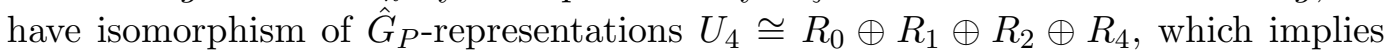
that $\mathbb{P}^{3}$ contains exactly 3 different points besides $P \in \mathbb{P}^{3}$, say $P_{1}, P_{2}$ and $P_{3}$, that are fixed by the group $G_{P}$. There is a unique subgroup $H \subset G$ such that $\mathbb{Z}_{7} \rtimes \mathbb{Z}_{3} \cong H \supset G_{P}$, and we may assume that the point $P_{1}$ is $H$-invariant (that is, corresponds to the subrepresentation $R_{0}$ ). Then its $G$-orbit consists of eight points. Thus, we see that $\left\{P, P_{2}, P_{3}\right\}$ is a $H$-orbit, which implies that the orbit $\Sigma$ exists and it is unique.

Suppose that $|\Sigma|=28$. Then $G_{P} \cong \mathrm{S}_{3}$. The action of $G_{P}$ on $\mathbb{P}^{3}$ is induced by a four-dimensional representation of $2 . \mathrm{S}_{3}$. By Lemma B.9, this representation splits as a sum of an irreducible two-dimensional representation and two non-isomorphic one-dimensional representations. Thus, there is a unique point $P^{\prime} \in \mathbb{P}^{3}$ such that $P \neq P^{\prime}$ and $P^{\prime}$ is fixed by $G_{P}$. On the other hand, the group $G$ contains exactly 28 subgroups isomorphic to $\mathrm{S}_{3}$. Moreover, it easily follows from Lemma B.9 that two different subgroups in $G$ isomorphic to $\mathrm{S}_{3}$ generates either a subgroup isomorphic to $\mathrm{S}_{4}$ or the whole group $G$. Thus, it follows from Lemma B.9 that no two of these 28 subgroups isomorphic to $\mathrm{S}_{3}$ can fix one point in $\mathbb{P}^{3}$. Thus, there are exactly two $G$-orbits in $\mathbb{P}^{3}$ consisting of 28 points.

Let $\Sigma_{8}, \Sigma_{24}$ and $\Sigma_{28} \neq \Sigma_{28}^{\prime}$ be $G$-orbits in $\mathbb{P}^{3}$ consisting of 8,24 and 28 points, respectively.

The group $\hat{G}$ naturally acts on $\mathbb{C}\left[x_{1}, x_{2}, x_{3}, x_{4}\right]$. Put $a=x_{2} x_{3} x_{4}, b=x_{2}^{3} x_{3}+x_{3}^{3} x_{4}+x_{4}^{3} x_{2}, c=x_{2}^{2} x_{3}^{3}+x_{3}^{2} x_{4}^{3}+x_{4}^{2} x_{2}^{3}, d=a^{2}+x_{2} x_{3}^{5}+x_{3} x_{4}^{5}+x_{4} x_{2}^{5}$, and $e=7 a b+x_{2}^{7}+x_{3}^{7}+x_{4}^{7}$. Furthermore, put $\Phi_{4}=2 x_{1}^{4}+6 a x_{1}+b$ and

$$
\begin{gathered}
\Phi_{6}=8 x_{1}^{6}-20 a x_{1}^{3}-10 b x_{1}^{2}-10 c x_{1}-14 a^{2}-d, \\
\Phi_{8}=x_{1}^{8}-2 a x_{1}^{5}+b x_{1}^{4}+2 c x_{1}^{3}+\left(6 a^{2}+d\right) x_{1}^{2}+2 a b x_{1}+a c, \\
\Phi_{8}^{\prime}=x_{1}^{8}+14 a x_{1}^{5}-7 b x_{1}^{4}+14 c x_{1}^{3}-7 d x_{1}^{2}+e x_{1}, \\
\Phi_{14}=48 x_{1}^{14}+168 a x_{1}^{11}+308 b x_{1}^{10}-1596 c x_{1}^{9}+126\left(42 a^{2}+11 d\right) x_{1}^{8}- \\
-8(37 e+490 a b) x_{1}^{7}+196\left(12 a c+5 b^{2}\right) x_{1}^{6}+196(15 a d-13 b c) x_{1}^{5}+ \\
+14\left(182 c^{2}-86 a e-7 b d\right) x_{1}^{4}+28(11 b e-42 c d) x_{1}^{3}+14\left(21 d^{2}-16 c e\right) x_{1}^{2}+14 d e x_{1}-e^{2} .
\end{gathered}
$$


Theorem 3.3 ([15], [25, Theorem 1]). The forms $\Phi_{4}, \Phi_{6}, \Phi_{8}, \Phi_{8}^{\prime}$ and $\Phi_{14}$ are $\hat{G}$-invariant.

Remark 3.4. There are no $\hat{G}$-invariant two-dimensional vector subspaces in $\mathbb{C}\left[x_{1}, x_{2}, x_{3}, x_{4}\right]$ that consist of linear, quadratic, cubic, quartic, quintic or sextic forms (see [11, Appendix 1]).

Let $F_{i}$ be a surface in $\mathbb{P}^{3}$ that is given by the equation

$$
\Phi_{i}\left(x_{1}, x_{2}, x_{3}, x_{4}\right)=0 \subset \mathbb{P}^{3} \cong \operatorname{Proj}\left(\mathbb{C}\left[x_{1}, x_{2}, x_{3}, x_{4}\right]\right),
$$

and let $F_{8}^{\prime}$ be a surface in $\mathbb{P}^{3}$ that is given by $\Phi_{8}^{\prime}\left(x_{1}, x_{2}, x_{3}, x_{4}\right)=0$.

Theorem 3.5 ([25, Theorem 1]). There are no $G$-invariant odd degree surfaces in $\mathbb{P}^{3}$, there are no $G$-invariant quadric surfaces in $\mathbb{P}^{3}$, and the only $G$-invariant quartic surface in $\mathbb{P}^{3}$ is the surface $F_{4}$.

One can check that the surface $F_{4}$ is smooth.

Lemma 3.6 (cf. [25, Theorem 1]). The sets $F_{4} \cap F_{6} \cap F_{8}^{\prime}, F_{4} \cap F_{6} \cap F_{14}$, and $F_{4} \cap F_{8}^{\prime} \cap F_{14}$ are finite.

Proof. This follows from explicit computations. We used the Magma software [2] to carry them out.

There is a $G$-invariant irreducible smooth curve $C_{6} \subset \mathbb{P}^{3}$ of genus 3 and degree 6 such that $\Sigma_{24}=C_{6} \cap F_{4}$, and $C_{6}$ is an intersection of cubic surfaces in $\mathbb{P}^{3}$ (see [15, page 154], [11, Example 2.8]).

Lemma 3.7. Let $C$ be a $G$-invariant curve in $\mathbb{P}^{3}$ such that $\operatorname{deg}(C) \leqslant 6$. Then $C=C_{6}$.

Proof. By Corollary B.2, we may assume that the curve $C$ is irreducible. If the curve $C$ is singular, then $|\operatorname{Sing}(C)| \geqslant 8$ by Lemma 3.2 , which easily leads to a contradiction by applying Lemma 2.15 to the normalization of the curve $C$. Then $C$ is smooth. Since $U_{4}$ is an irreducible representation of the group $\hat{G}$, the curve $C$ is not contained in a plane in $\mathbb{P}^{3}$. Then $C$ is a curve of genus 3 and degree 6 by Theorem 2.14 and Lemma 2.16. By Theorem 2.19, there is a unique $G$-invariant line bundle of degree 6 on the curve $C$, which implies that the embedding $C \hookrightarrow \mathbb{P}^{3}$ is unique up to the action of the group Aut ${ }^{G}\left(\mathbb{P}^{3}\right)$. But $\operatorname{Aut}^{G}\left(\mathbb{P}^{3}\right)=G$ by Remark 3.1 . which implies that $C=C_{6}$.

Note that $C_{6} \cap \Sigma_{8}=\varnothing$ by Lemma 2.15 ,

Lemma 3.8. There is a non-biregular involution $\tau \in \operatorname{Bir}^{G}\left(\mathbb{P}^{3}\right)$ such that the diagram

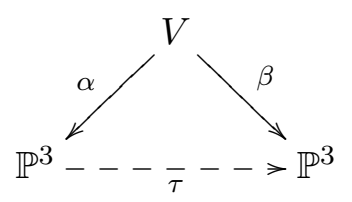

commutes and $\langle G, \tau\rangle \cong G \times \mathbb{Z}_{2}$, where $\alpha$ and $\beta$ are blow ups of the curve $C_{6}$.

Proof. The existence of the commutative diagram (3.9) is well-known (see [11, Remark 6.8]), the isomorphism $\langle G, \tau\rangle \cong \mathrm{PSL}_{2}\left(\mathbb{F}_{7}\right) \times \mathbb{Z}_{2}$ follows from the last three lines of the proof of [11, Lemma 6.4].

Let us introduce a $G$-invariant curve in $\mathbb{P}^{3}$, which has never been mentioned in the literature. 
Lemma 3.10. There is a $G$-invariant irreducible curve $C_{14} \subset \mathbb{P}^{3}$ of degree 14 such that $\Sigma_{8} \subset C_{14}$.

Proof. Let $\mathfrak{C}$ be the genus 3 curve introduced in Example 1.8. Then Aut $(\mathfrak{C}) \cong G$, which implies that $\mathfrak{C}$ admits a natural action of the group $G$. It follows from Theorem 2.19 that $\operatorname{Pic}^{G}(\mathfrak{C})=\langle\theta\rangle$, where $\theta$ is a $G$-invariant line bundle of degree 2. By [11, Lemma 6.4], there exists an isomorphism $H^{0}\left(\mathcal{O}_{\mathfrak{C}}(7 \theta)\right) \cong U_{4} \oplus U_{8}$, where $U_{8}$ is an irreducible eight-dimensional representation of the group $\hat{G}$ (see Appendix $\mathrm{B}$ ).

The linear system $|7 \theta|$ gives a $G$-equivariant embedding $\rho: \mathfrak{C} \hookrightarrow \mathbb{P}^{11}$ such that there exist unique $G$-invariant linear subspaces $\Pi_{3}$ and $\Pi_{7}$ in $\mathbb{P}^{11}$ of dimensions 3 and 7 , respectively. Then $\rho(\mathfrak{C}) \cap \Pi_{7}=\varnothing$ by Lemma 2.15 , Let $\iota: \mathbb{P}^{11} \rightarrow \Pi_{3}$ be a $G$-equivariant projection from $\Pi_{7}$, put $C_{14}=\iota \circ \rho(\mathfrak{C})$, and identify $\Pi_{3}$ with our $\mathbb{P}^{3}$. Then $C_{14} \subset \mathbb{P}^{3}$ is an irreducible $G$-invariant curve of degree 14 .

Let $H$ be subgroup in $G$ such that $H \cong \mathbb{Z}_{7} \rtimes \mathbb{Z}_{3}$. Then there is a $H$-invariant subset $\Sigma_{3} \subset \mathfrak{C}$ such that $\left|\Sigma_{3}\right|=3$. Note that $\Sigma_{3}$ is a subset of the $G$-orbit of length 24 , which implies that $\Sigma_{8} \subset C_{14}$ if and only if $\left|\iota \circ \rho\left(\Sigma_{3}\right)\right|=1$ by Lemma 3.2 . Let us show that $\iota \circ \rho\left(\Sigma_{3}\right)$ consists of a single point.

Let $T$ be a vector subspace in $H^{0}\left(\mathcal{O}_{\mathfrak{C}}(7 \theta)\right)$ that consists of sections vanishing at the subset $\Sigma_{3}$, and let $L_{i}$ be a $\hat{G}$-subrepresentation in $H^{0}\left(\mathcal{O}_{\mathfrak{C}}(7 \theta)\right)$ such that $L_{i} \cong U_{i}$ for $i \in\{4,8\}$. Then $\left|\iota \circ \rho\left(\Sigma_{3}\right)\right|=1$ if and only if $\operatorname{dim}\left(L_{4} \cap T\right)=3$ by the construction of the map $\iota \circ \rho$. Let us show that $\operatorname{dim}\left(L_{4} \cap T\right)=3$.

Take a subgroup $\hat{H} \subset \hat{G}$ such that $\phi(\hat{H})=H$. Then it follows from Lemma B.7 that $L_{8} \cong V_{3} \oplus V_{3}^{\prime} \oplus V_{1}^{\prime} \oplus V_{1}^{\prime \prime}$ and $L_{4} \cong V_{3} \oplus V_{1}$ as representations of the group $\hat{H}$, where $V_{3}, V_{3}^{\prime}, V_{1}, V_{1}^{\prime}, V_{1}^{\prime \prime}$ are different irreducible representations of dimensions $3,3,1,1,1$, respectively. Thus, there is an isomorphism $T \cong V_{3} \oplus V_{3} \oplus V_{3}^{\prime}$, because $T$ does not contain one-dimensional $\hat{H}$-subrepresentations, since the curve $\mathfrak{C}$ does not contain $H$-invariant subsets consisting of $\operatorname{deg}(7 \theta)-3=11$ points. Hence $L_{4} \cap T \cong V_{3}$.

Let us denote the points in $\Sigma_{8}$ by $O_{1}, O_{2}, \ldots, O_{8}$, and let us denote by $\mathcal{Q}$ the linear system of quadric surfaces in $\mathbb{P}^{3}$ that pass through $\Sigma_{8}$.

Example 3.11. The log pair $\left(\mathbb{P}^{3}, 2 \mathcal{Q}\right)$ is canonical. But $\operatorname{NLCS}\left(\mathbb{P}^{3}, 4 \mathcal{Q}\right)=\Sigma_{8}$.

Let $\pi: U \rightarrow \mathbb{P}^{3}$ be the blow up of the subset $\Sigma_{8}$, let $E_{i}$ be the exceptional divisor of the birational morphism $\pi$ such that $\pi\left(E_{i}\right)=O_{i}$ for every $i$. Then there is a commutative diagram

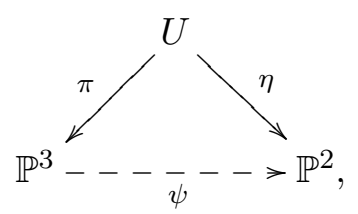

where $\psi$ is a rational map that is given by $\mathcal{Q}$, and $\eta$ is an elliptic fibration.

Lemma 3.13. The curve $C_{14}$ has an ordinary triple point at every point of the set $\Sigma_{8}$, the proper transform of the curve $C_{14}$ on the threefold $U$ is smooth, the curve $C_{14}$ is smooth outside of the points of the set $\Sigma_{8}$, the map $\psi: \mathbb{P}^{3} \rightarrow \mathbb{P}^{2}$ induces a birational map $C_{14} \rightarrow \psi\left(C_{14}\right)$, the curve $\psi\left(C_{14}\right)$ is a smooth curve of genus 3 and degree 4 , the intersection $C_{6} \cap C_{14}$ is empty.

Proof. Let $\bar{C}_{14}$ and $\bar{Q}$ be the proper transforms of the curve $C_{14}$ and a general surface in $\mathcal{Q}$ on the threefold $U$, respectively. Then mult $_{O_{i}}\left(C_{14}\right) \geqslant\left|\bar{C}_{14} \cap E_{i}\right| \geqslant 3$ 
by Lemma 2.11, Thus

$4 \geqslant 28-8\left|\bar{C}_{14} \cap E_{i}\right| \geqslant 2 \operatorname{deg}\left(C_{14}\right)-\sum_{i=1}^{8} E_{i} \cdot \bar{C}_{14}=\left(\pi^{*}(H)-\sum_{i=1}^{8} E_{i}\right) \bar{C}_{14}=\bar{Q} \cdot \bar{C}_{14} \geqslant 0$,

which implies that $\bar{C}_{14}$ has an ordinary triple point at every point of the set $\Sigma_{8}$. Since $4=2 \operatorname{deg}\left(C_{14}\right)-24=\bar{Q} \cdot \bar{C}_{14}$, we see that $\eta\left(\bar{C}_{14}\right)$ is a smooth curve of genus 3 and degree 4 by Lemma B.5. Therefore $\bar{C}_{14} \cong \eta\left(\bar{C}_{14}\right)$, which implies that $C_{14}$ is smooth outside of the points of the set $\Sigma_{8}$.

Let us show that $C_{6} \cap C_{14}=\varnothing$. Suppose that $C_{6} \cap C_{14} \neq \varnothing$. Then $\left|C_{6} \cap C_{14}\right| \geqslant 56$ by Lemma 2.16, since $\Sigma_{8} \not \subset C_{6}$. Let $S$ be a general cubic surface in $\mathbb{P}^{3}$ such that $C_{6} \subset S$. Then

$$
42=S \cdot C_{14} \geqslant \sum_{O \in C_{6} \cap C_{14}} \operatorname{mult}_{O}(S) \operatorname{mult}_{O}\left(C_{14}\right) \geqslant 56,
$$

because $C_{14} \not \subset S$. Thus, the intersection $C_{6} \cap C_{14}$ is empty.

Lemma 3.14. Let $C$ be a $G$-invariant curve such that $\operatorname{deg}(C) \leqslant 15$ and $\Sigma_{8} \subset C$. Then $C=C_{14}$.

Proof. Let $\bar{C}$ and $\bar{Q}$ be the proper transforms of the curve $C$ and a general surface in $\mathcal{Q}$ on the threefold $U$, respectively. Then $\operatorname{mult}_{O_{i}}(C) \geqslant\left|\bar{C} \cap E_{i}\right| \geqslant 3$ by Lemma2.11, Then

$$
6 \geqslant 30-8\left|\bar{C} \cap E_{i}\right| \geqslant 2 \operatorname{deg}(C)-\sum_{i=1}^{8} E_{i} \cdot \bar{C}=\left(\pi^{*}(H)-\sum_{i=1}^{8} E_{i}\right) \bar{C}=\bar{Q} \cdot \bar{C} \geqslant 0,
$$

which implies that $E_{i} \cdot \bar{C}=\left|\bar{C} \cap E_{i}\right|=3$.

We may assume that $C$ is a $G$-orbit of an irreducible curve $\Gamma \subset \mathbb{P}^{3}$. Then

$$
6 \geqslant 2 \operatorname{deg}(C)-24=\bar{Q} \cdot \bar{C}=\delta \operatorname{deg}(\eta(\bar{C}))
$$

for some positive integer $\delta$. We may assume that $\delta=1$ if $\bar{C}$ is contracted by $\eta$. Thus, we have $\operatorname{deg}(C) \in\{12,13,14,15\}$, which implies that either $\operatorname{deg}(\Gamma) \leqslant 2$ or $\Gamma=C$ (see Corollary B.2).

By Lemma B.5. we have $\operatorname{deg}(\psi(C)) \notin\{1,2,3\}$. Then $\operatorname{deg}(C) \in\{12,14,15\}$ and $\delta=1$. But

$$
6 \geqslant 2 \operatorname{deg}(C)-24=\operatorname{deg}(\eta(\bar{C}))
$$

which implies that $\operatorname{deg}(\eta(\bar{C})) \in\{0,4,6\}$ by Lemma B.5.

Recall that $G$-invariant quartic curve and sextic curve in $\mathbb{P}^{2}$ are irreducible (see Lemma B.5), which easily implies using (3.15) that either $\operatorname{deg}(\Gamma)=1$ and $\operatorname{deg}(C)=12$, or $\Gamma=C$.

If $\operatorname{deg}(\Gamma)=1$ and $\operatorname{deg}(C)=12$, then it follows from (3.15) that $|\eta(\bar{C})| \leqslant 12$, which is impossible, since $G$-orbit of every point in $\mathbb{P}^{2}$ consists of at least 21 points by Lemma B.6.

We see that $\Gamma=C$. Then $\bar{C}$ is not contracted by $\eta$, since there is no $G$-invariant point in $\mathbb{P}^{2}$.

Suppose that $\operatorname{deg}(C)=15$. Then $\bar{C} \cong \psi(C)$ and $\operatorname{deg}(\psi(C))=6$, which immediately implies that $\psi(C)$ is a smooth curve of genus 10 by Lemma B.5. Then there is a natural monomorphism

$$
U_{4} \cong H^{0}\left(\mathcal{O}_{U}\left(\pi^{*}(H)\right)\right) \hookrightarrow H^{0}\left(\mathcal{O}_{\bar{C}} \otimes \mathcal{O}_{U}\left(\pi^{*}(H)\right)\right) \cong \mathbb{C}^{6},
$$


which contradicts Lemma 2.15, since $\mathrm{SL}_{2}\left(\mathbb{F}_{7}\right)$ has no irreducible two-dimensional representations.

We see that $\operatorname{deg}(C)=14$. Thus $\bar{C} \cong \psi(C)$, which implies that $\bar{C}$ is a smooth curve of genus 3. Arguing as in the proof of Lemma 3.10, we see that $C=\sigma\left(C_{14}\right)$ for some $\sigma \in \mathrm{Aut}^{G}\left(\mathbb{P}^{3}\right)$. But Aut ${ }^{G}\left(\mathbb{P}^{3}\right)=G$ by Remark 3.1. Hence, we must have $C=C_{14}$, since the curve $C_{14}$ is $G$-invariant.

Lemma 3.16. Let $\mathcal{D}$ be the linear system consisting of all quintic surfaces in $\mathbb{P}^{3}$ that contain $C_{14}$. Then $\mathcal{D}$ is not empty and does not have fixed components, a general surface in $\mathcal{D}$ has a double point in every point of the set $\Sigma_{8}$, all curves contained in the base locus of the linear system $\mathcal{D}$ are disjoint from $C_{14}$, a general surface in $\mathcal{D}$ is smooth in a general point of the curve $C_{14}$, any two general surfaces in $\mathcal{D}$ are not tangent to each other along the curve $C_{14}$.

Proof. Let $\bar{C}_{14}$ be the proper transform of the curve $C_{14}$ on the threefold $U$, and let $\mathcal{I}$ be the ideal sheaf of the curve $\bar{C}_{14}$. Put $R=\pi^{*}(5 H)-\sum_{i=1}^{8} E_{i}$. Then

$h^{0}\left(\mathcal{O}_{U}(R) \otimes \mathcal{I}\right) \geqslant h^{0}\left(\mathcal{O}_{U}(R)\right)-h^{0}\left(\mathcal{O}_{\bar{C}_{14}} \otimes \mathcal{O}_{U}(R)\right)=48-h^{0}\left(\mathcal{O}_{\bar{C}_{14}} \otimes \mathcal{O}_{U}(R)\right)=4$, which implies that $\operatorname{dim}(\mathcal{D}) \geqslant 3$.

Let $D$ be a general surface in $\mathcal{D}$. Then $D$ is irreducible, because the linear system $\mathcal{D}$ does not have fixed components by Theorem 3.5 and $\mathcal{D}$ is not composed of a pencil by Remark 3.4 .

Let $\bar{D}$ be its proper transform of the surface $D$ on the threefold $U$, and let $\Gamma$ be a general fiber of the elliptic fibration $\eta$. Then

$$
20-8 \text { mult }_{O_{1}}(\mathcal{D})=\left(\pi^{*}(5 H)-\sum_{i=1}^{8} \operatorname{mult}_{O_{i}}(\mathcal{D}) E_{i}\right) \cdot \Gamma=\bar{D} \cdot \Gamma \geqslant 0
$$

which implies that mult $_{O_{1}}(\mathcal{D}) \leqslant 2$. Thus $\operatorname{mult}_{O_{1}}(\mathcal{D})=2$ by Lemma 2.11.

Let $D^{\prime}$ be a general surface in $\mathcal{D}$ such that $D \neq D^{\prime}$. Hence there is $\mu \in \mathbb{Z}_{>0}$ such that $D \cdot D^{\prime}=\mu C_{14}+B+Z$, where $Z$ is a curve not contained in the base locus of the linear system $\mathcal{D}$, and $B$ is a curve contained in the base locus of the linear system $\mathcal{D}$ such that $C_{14} \nsubseteq \operatorname{Supp}(B)$. Then

$$
25=\operatorname{deg}\left(D \cdot D^{\prime}\right)=14 \mu+\operatorname{deg}(B)+\operatorname{deg}(Z) \geqslant 20+\operatorname{deg}(Z)
$$

by Lemma 3.7. Thus, we see that $\mu=1$ and $\operatorname{deg}(B)+\operatorname{deg}(Z) \leqslant 11$, which implies, in particular, that the surfaces $D$ and $D^{\prime}$ are not tangent to each other along $C_{14}$, since $\mu=1$.

Note that $B$ is $G$-invariant, because $\mathcal{D}$ is $G$-invariant. Therefore $\operatorname{deg}(B) \geqslant 6$ by Lemma 3.7.

If the base locus of the linear system $\mathcal{D}$ consists of the curve $C_{14}$, then $C_{14}$ is a scheme-theoretic intersection of surfaces in $\mathcal{D}$ outside some finite subset of the curve $C_{14}$, because $\mu=1$.

Let $\bar{Z}$ and $\bar{B}$ be the proper transforms of the curves $Z$ and $B$ on the threefold $U$, respectively, and let $\bar{Q}$ be a proper transform of a general quadric surface in $\mathcal{Q}$ on the threefold $U$. Then

$$
\begin{aligned}
0 \leqslant \bar{Q} \cdot \bar{B}=2 \operatorname{deg}(B) & -\sum_{i=1}^{8} E_{i} \cdot \bar{B} \leqslant \\
& \leqslant 22-8\left|\operatorname{Supp}(\bar{B}) \cap E_{i}\right| \leqslant 22-8 \times\left\{\begin{array}{l}
3 \text { if } O_{i} \in \operatorname{Supp}(B), \\
0 \text { if } O_{i} \notin \operatorname{Supp}(B),
\end{array}\right.
\end{aligned}
$$


which implies that $\Sigma_{8} \cap \operatorname{Supp}(B)=\varnothing$. But

$$
3+\operatorname{mult}_{O_{i}}(Z)=\operatorname{mult}_{O_{i}}\left(C_{14}\right)+\operatorname{mult}_{O_{i}}(B)+\operatorname{mult}_{O_{i}}(Z)=\operatorname{mult}_{O_{i}}\left(D_{1} \cdot D_{2}\right) \geqslant 4,
$$

which implies that $\Sigma_{8} \subset \operatorname{Supp}(Z)$. Hence

$$
2 \operatorname{deg}(Z)-8 \geqslant 2 \operatorname{deg}(Z)-\sum_{i=1}^{8} E_{i} \cdot \bar{Z}=\bar{Q} \cdot \bar{Z} \geqslant 0,
$$

which implies that $\operatorname{deg}(Z) \geqslant 4$, and $\operatorname{deg}(Z)=4$ if and only if $\bar{Z}$ is contracted by $\eta$. But $10 \leqslant 6+\operatorname{deg}(Z) \leqslant \operatorname{deg}(B)+\operatorname{deg}(Z) \leqslant 11$, which implies that either $\operatorname{deg}(B)=7$ and $\operatorname{deg}(Z)=4$, or $\operatorname{deg}(B)=6$ and $\operatorname{deg}(Z)=5$.

If $\operatorname{deg}(Z)=4$, then $\bar{D}$ is contracted by the morphism $\eta$ to a curve of degree $d$, then $\bar{D} \sim d\left(\pi^{*}(2 H)-\sum_{i=1}^{8} E_{i}\right)$, which is a contradiction. Thus, we see that $\operatorname{deg}(B)=6$ and $\operatorname{deg}(Z)=5$.

By Lemma 3.7, we have $B=C_{6}$. Therefore $C_{6} \cap C_{14}=\varnothing$ by Lemma 3.13.

Let us study some properties of the subset $\Sigma_{28} \subset \mathbb{P}^{3}$, which also hold for $\Sigma_{28}^{\prime}$.

Lemma 3.17. Let $Z$ be a $G$-invariant curve in $\mathbb{P}^{3}$ that contains $\Sigma_{28}$. Then $\operatorname{deg}(Z) \geqslant 16$ if the set $\Sigma_{28}$ imposes independent linear conditions on quartic surfaces in $\mathbb{P}^{3}$.

Proof. Suppose that the set $\Sigma_{28}$ imposes independent linear conditions on quartic surfaces in $\mathbb{P}^{3}$, and suppose that $\operatorname{deg}(Z) \leqslant 15$. Let us derive a contradiction.

Without loss of generality, we may assume that $Z$ is a $G$-orbit of an irreducible curve $Z_{1}$.

Put $Z=\sum_{i=1}^{r} Z_{i}$, where $Z_{i}$ is an irreducible curve in $\mathbb{P}^{3}$ and $r \in \mathbb{Z}_{>0}$. Then $\operatorname{deg}(Z)=r \operatorname{deg}\left(Z_{i}\right) \leqslant 15$, which implies that $r \in\{1,7,8,14\}$ by Corollary B.2. Thus, if $r \neq 1$, then $\operatorname{deg}\left(Z_{i}\right) \in\{1,2\}$.

If $\operatorname{deg}\left(Z_{1}\right)=2$ and $r \neq 1$, then $r=7$, which contradicts Lemma 3.2. Hence $Z_{1}$ is a line if $r \neq 1$.

Let $\Gamma$ be the stabilizer subgroup in $G$ of the curve $Z_{1}$. If $r=7$, then $\Gamma \cong \mathrm{S}_{4}$, which implies that $\left|Z_{1} \cap \Sigma_{28}\right| \geqslant 6$, which is impossible, since $\Sigma_{28}$ impose independent linear conditions on quartic surfaces.

Let $\hat{\Gamma}$ be the smallest subgroup of the group $\hat{G}$ such that $\phi(\hat{\Gamma})=\Gamma$. Then

- if $r=8$, then $\hat{\Gamma} \cong 2 .\left(\mathbb{Z}_{7} \rtimes \mathbb{Z}_{3}\right)$, which contradicts Lemma B.7.

- if $r=14$, then $\hat{\Gamma} \cong 2 . \mathrm{A}_{4}$, which contradicts Lemma B.9.

Thus, we see that the curve $Z$ is irreducible.

By Lemma 2.15, the curve $Z$ must be singular at every point of the set $\Sigma_{28}$, which implies that $Z \neq C_{14}$ by Lemma 3.13. Hence $\Sigma_{8} \not \subset Z$ by Lemma 3.14.

Let $A$ be a point in $\Sigma_{28}$. Then there exists a quartic surface $S \subset \mathbb{P}^{3}$ such that $S$ contain all points of the set $\Sigma_{28} \backslash\{A\}$ and the surface $S$ does not contain the point $A$. Therefore

$$
4 \operatorname{deg}(Z)=S \cdot Z \geqslant \sum_{O \in \Sigma_{28} \backslash\{A\}} \operatorname{mult}_{O}(Z) \geqslant \sum_{O \in \Sigma_{28} \backslash\{A\}} 2 \geqslant 54
$$

which implies that $\operatorname{deg}(Z) \geqslant 14$. Thus, either $\operatorname{deg}(Z)=14$ or $\operatorname{deg}(Z)=15$.

If $\operatorname{deg}(Z)=15$, then $Z \subset F_{4} \cap F_{6} \cap F_{8}^{\prime}$ by Lemma 2.15, since 60, 90 and 120 are not equal to $24 n_{1}+42 n_{2}+56 n_{3}+84 n_{4}+168 n_{5}$ for any non-negative integers $n_{1}$, $n_{2}, n_{3}, n_{4}$ and $n_{5}$. Then $\operatorname{deg}(Z)=14$ by Lemma 3.6. 
Let $\bar{Z}$ be the normalization of the curve $Z$, and let $g$ be the genus of the curve $\bar{Z}$. Then

$$
g \leqslant 66-|\operatorname{Sing}(Z)| \leqslant 38
$$

because the projection from a general points of the curve $Z$ gives us a birational isomorphism between $Z$ and a plane curve of degree 13 with at least $|\operatorname{Sing}(Z)|$ singular points.

Note that $G$ naturally acts on both curves $Z$ and $\bar{Z}$. Let us show that $Z \backslash \Sigma_{28}$ is smooth.

Suppose that $\operatorname{Sing}(Z) \neq \Sigma_{28}$. Let $\Lambda$ be a $G$-orbit of a point in $\operatorname{Sing}(Z) \backslash \Sigma_{28}$. Then $|\Lambda| \leqslant 66-\left|\Sigma_{28}\right|=38$ by (3.18), and $|\Lambda| \in\{24,28\}$ by Lemma 3.2. If $\Lambda=C_{6} \cap F_{4}$, then

$$
42=S \cdot Z \geqslant \sum_{O \in \Lambda} \operatorname{mult}_{O}(S) \operatorname{mult}_{O}(Z) \geqslant 48
$$

where $S$ is a general cubic surface such that $C_{6} \subset S$. Thus, we see that $|\Lambda| \neq 24$ by Lemma 3.2, which implies that $|\Lambda|=28$. Therefore, it follows from (3.18) and Lemma 2.16 that $g \in\{3,8,10\}$ and the points of the set $\Lambda \cup \Sigma_{28}$ must be singular points of the curve $Z$ of multiplicity two, which implies that $\bar{Z}$ has at least two $G$-orbits consisting of 56 points, which contradicts Lemma 2.16.

Thus, we see that $Z$ is smooth outside of the set $\Sigma_{28}$.

Let $B$ be a sufficiently general point of the curve $Z$, let $\mathcal{M}$ be a linear system consisting of all quartic surfaces in $\mathbb{P}^{3}$ that contain the set $\Sigma_{28} \cup B$. Then one has $\operatorname{dim}(\mathcal{M}) \geqslant 35-29=6$.

Let $M$ be a general surface in $\mathcal{M}$. If $Z \not \subset M$, then

$$
56=M \cdot Z \geqslant \operatorname{mult}_{B}(M) \operatorname{mult}_{B}(Z)+\sum_{O \in \Sigma_{28}} \operatorname{mult}_{O}(M) \operatorname{mult}_{O}(Z) \geqslant 57,
$$

which is a contradiction. Thus, the curve $Z$ is contained in the base locus of the linear system $\mathcal{M}$, which implies that the linear system $\mathcal{M}$ is $G$-invariant, because $Z$ is $G$-invariant.

The linear system $\mathcal{M}$ does not have fixed components by Theorem 3.5 and $\mathcal{M}$ is not composed of a pencil by Remark 3.4. This implies, in particular, that the surface $M$ is irreducible.

Let $M^{\prime}$ be another general surface in the linear system $\mathcal{M}$. Put $M \cdot M^{\prime}=\mu Z+\Upsilon$, where $\mu \in \mathbb{Z}_{>0}$, and $\Upsilon$ is an effective one-cycle such that $Z \not \subset \operatorname{Supp}(\Upsilon)$. Then

$$
16=\operatorname{deg}\left(M \cdot M^{\prime}\right)=14 \mu+\operatorname{deg}(\Upsilon)
$$

which implies that $\mu=1$, and the base locus of the linear system $\mathcal{M}$ contain no curves except $Z$, because there are no $G$-invariant lines or conics in $\mathbb{P}^{3}$. Thus mult $_{Z}(\mathcal{M})=1$ and $\operatorname{deg}(\Upsilon)=2$, which implies, in particular, that the surface $M$ is uniruled.

Let $M^{\prime \prime}$ be another general surface in the linear system $\mathcal{M}$. Then the intersection $M \cap M^{\prime} \cap M^{\prime \prime}$ consists of the curve $C$ and the intersection $\Upsilon \cap M^{\prime \prime}$, which immediately implies that the base locus of the linear system $\mathcal{M}$ consists of at most 8 points outside of the curve $Z$. Thus, it follows from Lemma 3.2 that either the base locus of the linear system $\mathcal{M}$ consists of the curve $Z$, or the base locus of the linear system $\mathcal{M}$ consists of the curve $Z$ and the set $\Sigma_{8}$. 
Let $Q$ be a general surface in the linear system $\mathcal{Q}$. If $\Sigma_{8} \subset M \cap M^{\prime}$, then

$$
\begin{aligned}
32=Q \cdot M \cdot M^{\prime}=Q \cdot Z+\sum_{O \in \Sigma_{8}} \operatorname{mult}_{O}(Q) \operatorname{mult}_{O}(\Upsilon) & = \\
& =28+\sum_{O \in \Sigma_{8}} \operatorname{mult}_{O}(Q) \operatorname{mult}_{O}(\Upsilon) \geqslant 36,
\end{aligned}
$$

which implies that $\Sigma_{8} \not \subset M \cap M^{\prime}$. Hence $Z$ is the base locus of the linear system $\mathcal{M}$.

By adjunction formula, we see that the singularities of the surface $M$ are not canonical, which implies that $\left(\mathbb{P}^{3}, \mathcal{M}\right)$ is not canonical by [22, Theorems 4.8.1 and 7.9] (cf. [22, Theorem 7.5]). But $Z \notin \mathbb{N} \mathbb{C S}\left(\mathbb{P}^{3}, \mathcal{M}\right)$, because $\operatorname{mult}_{Z}(\mathcal{M})=1$. Thus, the set $\mathbb{N} \mathbb{C S}\left(\mathbb{P}^{3}, \mathcal{M}\right)$ contains a point $P \in Z$. Thus

$$
\operatorname{mult}_{P}(\Upsilon) \geqslant 4-\operatorname{mult}_{P}(Z)=\left\{\begin{array}{l}
2 \text { if } P \in \Sigma_{28}, \\
3 \text { if } P \notin \Sigma_{28},
\end{array}\right.
$$

since $\operatorname{mult}_{P}(\mathcal{M}) \geqslant 2$. Let $\Theta$ be the $G$-orbit of the point $P$. Then $|\Theta| \geqslant 28$ by Lemma 3.2, and

$$
8=M \cdot M^{\prime} \cdot M^{\prime \prime}-M^{\prime \prime} \cdot Z=M^{\prime \prime} \cdot \Upsilon \geqslant \sum_{O \in \Theta} \operatorname{mult}_{O}\left(M^{\prime \prime}\right) \operatorname{mult}_{O}(\Upsilon) \geqslant 56,
$$

which is a contradiction.

Let $\mathcal{R}$ be a linear system consisting of all quartic surfaces in $\mathbb{P}^{3}$ that pass through $\Sigma_{28}$.

Lemma 3.19. If the set $\Sigma_{28}$ imposes independent linear conditions on quartic surfaces in $\mathbb{P}^{3}$, then every curve in the base locus of the linear system $\mathcal{R}$ contains no points of the set $\Sigma_{28}$.

Proof. Let $C$ be an irreducible curve in $\mathbb{P}^{3}$ such that $C$ is contained in the base locus of the linear system $\mathcal{R}$, let $Z$ be the $G$-orbit of the curve $C$, let $R_{1}$ and $R_{2}$ be general surfaces in $\mathcal{R}$. Put $R_{1} \cdot R_{2}=\mu Z+\Upsilon$, where $\mu \in \mathbb{Z}_{>0}$, and $\Upsilon$ is an effective one-cycle such that $C \not \subset \operatorname{Supp}(\Upsilon)$. Then $16=\operatorname{deg}\left(R_{1} \cdot R_{2}\right)=\mu \operatorname{deg}(Z)+\operatorname{deg}(\Upsilon)$, which implies that $\operatorname{deg}(Z) \leqslant 15$ by Remark 3.4. Now it follows from Lemma 3.17 that $\Sigma_{28} \not \subset Z$ and $C \cap \Sigma_{28}=\varnothing$ if the points of the set $\Sigma_{28}$ impose independent linear conditions on quartic surfaces in $\mathbb{P}^{3}$.

We do not know whether or not $\Sigma_{28}$ imposes independent linear conditions on quartic surfaces.

\section{Compactified moduli space of $(1,7)$-POlarized ABElian SURFACES}

Let $\mathfrak{C}$ be a curve in $\mathbb{P}^{2}$ that is given by the equation

$$
x^{4}+y^{4}+z^{4}+3 \epsilon\left(x^{2} y^{2}+x^{2} z^{2}+y^{2} z^{2}\right)=0 \subset \mathbb{P}^{2} \cong \operatorname{Proj}(\mathbb{C}[x, y, z]),
$$

where $\epsilon=-1 / 2+\sqrt{-7} / 2$. Then $\mathfrak{C}$ is isomorphic to the curve described in Example 1.8 (see [24, page 55]).

Remark 4.2. The Hessian curve of the curve $\mathfrak{C}$ is a smooth sextic curve (see [13, Example 6.1.1]).

Remark 4.3. The quartic $\mathfrak{C}$ is not degenerate (see [13, Definition 2.8]). To see this apply [13, Theorem 6.12.2] (keeping in mind [13, Definition 6.12.1]) and use [13, Corollary 6.6.3], [13, Example 6.7] and Remark 4.2 above. 
Put $\bar{\epsilon}=-1 / 2-\sqrt{-7} / 2$. Let $\psi: \mathrm{SL}_{3}(\mathbb{C}) \rightarrow \operatorname{Aut}\left(\mathbb{P}^{2}\right) \cong \mathrm{PGL}_{3}(\mathbb{C})$ be a natural projection. Put

$$
\begin{aligned}
A=\left(\begin{array}{ccc}
-1 & 0 & 0 \\
0 & 0 & -1 \\
0 & -1 & 0
\end{array}\right), B=\left(\begin{array}{lll}
0 & 1 & 0 \\
0 & 0 & 1 \\
1 & 0 & 0
\end{array}\right), \\
C=\left(\begin{array}{ccc}
0 & 1 & 0 \\
1 & 0 & 0 \\
0 & 0 & -1
\end{array}\right), D=\frac{1}{2}\left(\begin{array}{ccc}
-1 & 1 & \bar{\epsilon} \\
\epsilon & \epsilon & 0 \\
-1 & 1 & -\bar{\epsilon}
\end{array}\right),
\end{aligned}
$$

put $G=\psi(\langle A, B, C, D\rangle)$, and let $X=\operatorname{VSP}(\mathfrak{C}, 6)$ (see Definition A.3). By [24, p. 55], we have $\operatorname{Aut}(\mathfrak{C}) \cong G \cong\langle A, B, C, D\rangle \cong \mathrm{PSL}_{2}\left(\mathbb{F}_{7}\right)$.

Theorem 4.4 ([26, Theorem 4.4]). The threefold $X$ is a smooth Fano threefold such that $\operatorname{Pic}(X)=\mathbb{Z}\left[-K_{X}\right]$ and $\left(-K_{X}\right)^{3}=22$.

The action of the group $G$ on the plane $\mathbb{P}^{2}$ induces its natural action on the threefold $X$. Therefore, the vector space $H^{0}\left(\mathcal{O}_{X}\left(-K_{X}\right)\right)$ has a natural structure of a 14dimensional representation of the group $G$.

Theorem 4.5. In the notation of Appendix B, one has

$$
H^{0}\left(\mathcal{O}_{X}\left(-K_{X}\right)\right) \cong I \oplus W_{6} \oplus W_{7} .
$$

Proof. We may assume that $W \cong W_{3}$. By Theorem A.5, one has

$$
U_{7} \cong \operatorname{Sym}^{3}\left(W_{3}^{\vee}\right) / W_{3} \cong W_{7} \cong U_{7}^{\vee}
$$

where all isomorphisms are isomorphisms of $G$-representations. Hence, we have

$$
U_{14} \cong \Lambda^{3}\left(U_{7}\right) /\left(W_{3}^{\vee} \otimes U_{7}^{\vee}\right) \cong \Lambda^{3}\left(W_{7}\right) /\left(W_{3}^{\vee} \otimes W_{7}\right) \cong U_{14}^{\vee},
$$

which implies the required assertion by Corollary B.4.

Corollary 4.6. There is a unique $G$-invariant surface in $\left|-K_{X}\right|$.

Let us identify $X$ with its anticanonical image in $\mathbb{P}^{13}$ (see Appendix $\mathrm{A}$ ).

Lemma 4.7. Let $Q \subset X$ be a surface swept out by the lines contained in $X$. Then $Q \sim-2 K_{X}$, and $Q$ is irreducible.

Proof. By [34, the surface $Q$ is reduced and $Q \sim-2 K_{X}$. Suppose that $Q$ is reducible. Then $Q=Q_{1} \cup Q_{2}$, where $Q_{1}$ and $Q_{2}$ are irreducible surfaces such that $Q_{1} \neq Q_{2}$. Then $Q_{1} \sim Q_{2} \sim-K_{X}$, and both $Q_{1}$ and $Q_{2}$ must be $G$-invariant. On the other hand, there is a unique $G$-invariant surface in $\left|-K_{X}\right|$ is by Lemma 4.5, which is a contradiction.

In the remaining part of this section we are going to prove the following result.

Theorem 4.8. Let $\Sigma$ be a $G$-orbit of a point in $X$ such that $|\Sigma| \leqslant 20$. Then $|\Sigma| \in\{8,14\}$, and if $|\Sigma|=8$, then $\Sigma$ is unique.

Remark 4.9. There exist finitely many points in $X$ whose $G$-orbits consist of 14 points.

Before proving Theorem 4.8, let us use Theorem 4.8 to prove the following result.

Lemma 4.10. Let $F$ be the unique $G$-invariant surface in $\left|-K_{X}\right|$. Then $F$ is smooth. 
Proof. The minimal resolution of the surface $F$ admits a faithful action of the group $\mathrm{PSL}_{2}\left(\mathbb{F}_{7}\right)$, which implies that $F$ is smooth by [32, Claim 2.1] if it has at most canonical singularities. Let us show that $F$ has at most canonical singularities (Du Val singularities). It is well-known that $F$ has canonical singularities if and only if the surface $F$ has rational singularities (see [22, Theorem 1.11]). By Theorem 1.12, the log pair $(X, F)$ is log canonical.

Suppose that $F$ has worse than canonical singularities. Let $S$ be a minimal center in $\mathbb{L} \mathbb{C S}(X, F)$. Then $S \neq F$ by Theorem 2.10 , because $F$ has worse than canonical singularities. Hence, either $S$ is a curve or a point by Theorem 2.10 . Let $Z$ be the $G$-orbit of the subvariety $S$. Then $Z \subset F$.

Choose any $\epsilon \in \mathbb{Q}$ such that $2 \gg \epsilon>1$. Then arguing as in the proof of [20, Theorem 1.10], we can find a $G$-invariant $\mathbb{Q}$-divisor $D$ such that $D \sim_{\mathbb{Q}} \epsilon F$, the log pair $(X, D)$ is log canonical, and every minimal center in $\mathbb{L} \mathbb{C S}(X, D)$ is an irreducible component of the subvariety $Z$ (see Lemma 2.13).

Let $H$ be a sufficiently general hyperplane section of the threefold $X$, and let $\mathcal{I}_{Z}$ be the ideal sheaf of the subvariety $Z$. Then there is an exact sequence of $G$-representations

$$
0 \rightarrow H^{0}\left(\mathcal{O}_{X}(H) \otimes \mathcal{I}_{Z}\right) \rightarrow H^{0}\left(\mathcal{O}_{X}(H)\right) \rightarrow H^{0}\left(\mathcal{O}_{Z} \otimes \mathcal{O}_{X}(H)\right) \rightarrow 0
$$

by Theorem 2.2. Put $q=h^{0}\left(\mathcal{O}_{X}(H) \otimes \mathcal{I}_{Z}\right)$. One has

$$
h^{0}\left(\mathcal{O}_{Z} \otimes \mathcal{O}_{X}(H)\right)=14-q \in\{6,7,13\},
$$

because $q \in\{1,7,8\}$ by Theorem 4.5, since $Z \subset F$. Hence $S$ is not a point by Theorem 4.8.

We see that $S$ is a curve. Moreover, $S$ is a smooth curve of genus $g$ such that $-K_{X} \cdot S=\operatorname{deg}(S) \geqslant 2 g-1$ by Theorem 2.10, By Remark 2.12, the curve $Z$ is a disjoint union of smooth irreducible curves. Let $r$ be the number of connected components of the curve $Z$. Put $d=\operatorname{deg}(S)$. Then

$$
g \leqslant d-g+1 \leqslant r(d-g+1)=14-q \in\{6,7,13\}
$$

by (4.11), which implies that $d \leqslant 13+g \leqslant 27$.

Let us show that $r=1$. Suppose that $r \geqslant 2$. Then $r \geqslant 7$ and $r \neq 13$ by Corollary B.2. Thus $r=7$ and $d-g+1=1$ by (4.12), which implies that $d=g$. But $d \geqslant 2 g-1$, so that $d=g=1$, which is absurd.

We see that $r=1$. There is a natural faithful action of the group $G$ on the curve $S$. But $d=13+g-q \geqslant 2 g-1$, by (4.12), which implies that $g \leqslant 14-q$. Hence $g \in\{3,8,10\}$ by Lemma 2.16,

Let us show that $g=3$. Suppose that $g \neq 3$. Then $g \in\{8,10\}$. It follows from (4.12) that $q \leqslant 14-g \leqslant 6$, which implies that $q=1$, because $q \in\{1,7,8\}$. Hence, it follows from (4.12) that $d \in\{20,22\}$, which is impossible by Lemmas 2.20 and 2.21

Thus, we have $g=3$, so that $d=16-q$ by (4.12), where $q \in\{1,7,8\}$. By Theorem 2.19, there is a $G$-invariant line bundle $\theta \in \operatorname{Pic}(S)$ of degree 2 such that $\operatorname{Pic}^{G}(S)=\langle\theta\rangle$, which implies that $q=8$ and $\left.H\right|_{S} \sim 2 K_{S}$, because $d=16-q$. Thus $d=8$.

Let $Q$ be a surface in $X$ that is spanned by lines. Then $Q$ is $G$-invariant. If $S \not \subset Q$, then $|Q \cap S| \leqslant 2 \operatorname{deg}(S)=16$, which is impossible by Lemma 2.15, Thus, we see that $S \subset Q \cap F$. By Lemma 4.7, the surface $Q$ is irreducible, and $Q \sim-2 K_{X}$. Let $P$ be a general point in $S$. There exists a line $L \subset X$ such that $P \in L$. If $L \not \subset F$, 
then

$$
1=\operatorname{deg}(L)=F \cdot L \geqslant \operatorname{mult}_{P}(F) \operatorname{mult}_{P}(L) \geqslant \operatorname{mult}_{S}(F) \geqslant 2,
$$

which simply means $L \subset F$. Since $g \neq 0$, the generality of the point $P \in S$ implies that the surface $F$ is swept out by lines, which is impossible since $Q \not \subset F$.

Remark 4.13. Let $\Sigma$ be a $G$-invariant subset of the threefold $X$ such that we have $|\Sigma| \in\{8,14\}$, and let $F$ be the unique $G$-invariant surface in $\left|-K_{X}\right|$. Then $F$ is smooth by Lemma 4.10, Since $G$ acts symplectically on $F$, it follows from [42] that $\Sigma \cap F=\varnothing$.

Now we are going to prove Theorem 4.8, Put

$$
\square=X \backslash\left\{\Gamma \in \operatorname{Hilb}_{6}\left(\check{\mathbb{P}}^{2}\right) \mid \Gamma \text { is polar to the curve } C\right\} \subset \operatorname{Hilb}_{6}\left(\check{\mathbb{P}}^{2}\right) \text {. }
$$

Lemma 4.14. Let $\Sigma$ be a $G$-orbit in $\square$ such that $|\Sigma| \leqslant 20$. Then $|\Sigma|=8$ and $\Sigma$ is unique.

Proof. It follows from [28, $\S 2.3]$ that there exists an effective one-cycle

$$
m_{1} L_{1}+\ldots+m_{r} L_{r} \sim \mathcal{O}_{\mathbb{P}^{2}}(6)
$$

on $\mathbb{P}^{2}$ that corresponds to a point in $\Sigma$, where $L_{i}$ are lines in $\mathbb{P}^{2}$ and $m_{i}$ are positive integers.

Without loss of generality, we may assume that $m_{1} \geqslant \ldots \geqslant m_{r}$. Then

$$
\left(m_{1}, \ldots, m_{r}\right) \in\{(2,1,1,1,1),(3,1,1,1),(2,2,1,1),(2,2,2),(4,2)\}
$$

by [28, Theorem 1.1] (cf. [27, Theorem A]). Thus, it follows from $|\Sigma| \leqslant 20$ that $\left(m_{1}, \ldots, m_{r}\right)=(2,2,2)$, because the smallest $G$-invariant subset in $\mathbb{P}^{2}$ has at least 21 points by Lemma B.6.

Let us consider the lines $L_{1}, L_{2}, L_{3}$ as points in the dual projective plane $\check{\mathbb{P}}^{2}$ with a natural action of the group $G$, and let $\hat{\mathfrak{C}}$ be the unique $G$-invariant quartic curve in $\check{\mathbb{P}}^{2}$. Then $\hat{\mathfrak{C}} \cap\left\{L_{1}, L_{2}, L_{3}\right\} \neq \varnothing$ by [28, Proposition 3.13], which easily implies that $|\Sigma|=8$ and $\Sigma$ is unique by Lemma 2.16 .

Therefore, to prove Theorem 4.8 it is enough to consider only $G$-orbits of points in $X \backslash \square$, which are points in $X$ that can be represented by polar hexagons to the curve $\mathfrak{C}$.

Suppose that the assertion of Theorem 4.8 is false. Let us derive a contradiction.

By Lemmas 4.14 and B.1, there are six lines $L_{1}, \ldots, L_{6}$ on $\mathbb{P}^{2}$ such that

$$
F(x, y, z)=L_{1}^{4}(x, y, z)+\cdots+L_{6}^{4}(x, y, z),
$$

where $L_{i}(x, y, z)$ is a linear form such that $L_{i}$ is given by $L_{i}(x, y, z)=0$, and the stabilizer subgroup of the hexagon $\sum_{i=1}^{6} L_{i}$ in the group $G$ is isomorphic either to $\mathbb{Z}_{7} \rtimes \mathbb{Z}_{3}$ or to $\mathrm{S}_{4}$.

Remark 4.16. The lines $L_{1}, \ldots, L_{6}$ are distinct since the quartic $\mathfrak{C}$ is not degenerate (see Remark 4.3).

Lemma 4.17. Let $g \in G$ be an element of order 7 . Then $\sum_{i=1}^{6} L_{i}$ is not $g$-invariant.

Proof. Suppose that $\sum_{i=1}^{6} L_{i}$ is $g$-invariant. Then $g\left(L_{i}\right)=L_{i}$ for every $i \in\{1, \ldots, 6\}$. Take $M \in \mathrm{SL}_{3}(\mathbb{C})$ such that $\psi(M)=g^{-1}$ and $M^{7}=1$. Then $L_{i}((x, y, z) M)=$ $\lambda_{i} L_{i}(x, y, z)$ for some $\lambda_{i} \in \mathbb{C}$ for every $i \in\{1, \ldots, 6\}$, i. e. $\lambda_{1}, \ldots, \lambda_{6}$ are eigenvalues of the matrix $M$. 
Since $g$ has order 7 , we have $\lambda_{1}^{7}=\ldots=\lambda_{6}^{7}=1$. We may assume that

$$
\lambda_{1}=\lambda_{2}=\lambda_{3}=\lambda_{4}=\lambda_{5},
$$

since $L_{1}, \ldots, L_{6}$ are different lines. Then $\lambda_{6} \neq \lambda_{1}$ by (4.15). Thus one obtains $\left|L_{1} \cap L_{2} \cap L_{3} \cap L_{4} \cap L_{5}\right|=1$ and $L_{1} \cap L_{2} \cap L_{3} \cap L_{4} \cap L_{5} \notin L_{6}$. Therefore

$$
\sum_{i=1}^{6} L_{i}^{4}(x, y, z)=F((x, y, z) M)=\lambda_{6}^{4} L_{6}^{4}(x, y, z)+\lambda_{1}^{4} \sum_{i=1}^{5} L_{i}^{4}(x, y, z),
$$

by (4.15), which easily implies that $\lambda_{1}^{4}=\lambda_{6}^{4}=1$. Hence $\lambda_{1}=\lambda_{6}=1$, which is a contradiction.

Let $\Gamma$ be a stabilizer subgroup in $G$ of the hexagon $\sum_{i=1}^{6} L_{i}$. Then $\Gamma \cong \mathrm{S}_{4}$ by Lemma 4.17. We may assume that $\Gamma=\langle\psi(A), \psi(B), \psi(C)\rangle$, because $G$ has two subgroups isomorphic to $\mathrm{S}_{4}$ up to a conjugation (see Lemma B.1), which are switched by an outer automorphism in $\operatorname{Aut}(G)$ that can be realized as a complex conjugation in appropriate coordinates.

Lemma 4.18. The group $\Gamma$ acts transitively on the lines $L_{1}, L_{2}, L_{3}, L_{4}, L_{5}, L_{6}$.

Proof. Since the action of the group $\Gamma$ on $\mathbb{P}^{2}$ comes from an irreducible threedimensional representation of the group $\Gamma$, the $\Gamma$-orbit of every line in $\mathbb{P}^{2}$ contains at least 3 lines. But the only $\Gamma$-invariant 3 -tuple of lines in $\mathbb{P}^{2}$ is given by $x y z=0$.

Let $\Omega$ be a stabilizer subgroup in $\Gamma$ of the line $L_{1}$. Then either $\Omega \cong \mathbb{Z}_{2} \times \mathbb{Z}_{2}$ or $\Omega \cong \mathbb{Z}_{4}$.

Lemma 4.19. The group $\Omega$ is not isomorphic to $\mathbb{Z}_{4}$.

Proof. Suppose that $\Omega \cong \mathbb{Z}_{4}$. Then we may assume that $\Omega$ is generated $\psi(C B)$. One has

$$
C B=\left(\begin{array}{ccc}
0 & 0 & 1 \\
0 & 1 & 0 \\
-1 & 0 & 0
\end{array}\right)
$$

and the eigenvalues of the matrix $C B$ are 1 and $\pm \sqrt{-1}$.

Note that $(0,1,0)$ is the eigenvector of the matrix $C B$ that corresponds to the eigenvalue 1 , and $(\mp \sqrt{-1}, 0,1)$ is its eigenvector that corresponds to the eigenvalue $\pm \sqrt{-1}$. Thus $L_{1}(x, y, z)=\mu(x \pm \sqrt{-1} z)$, where $\mu$ is a non-zero complex number. The hexagon $\sum_{i=1}^{6} L_{i}$ is given by

$$
(x-\sqrt{-1} z)(x+\sqrt{-1} z)(y+\sqrt{-1} x)(y-\sqrt{-1} x)(z+\sqrt{-1} y)(z-\sqrt{-1} y)=0,
$$

which implies that the quartic form $x^{4}+y^{4}+z^{4}+3 \epsilon\left(x^{2} y^{2}+x^{2} z^{2}+y^{2} z^{2}\right)$ is equal to

$$
\begin{aligned}
\mu_{1}(x-\sqrt{-1} z)^{4}+\mu_{2} & (x+\sqrt{-1} z)^{4}+\mu_{3}(y+\sqrt{-1} x)^{4}+ \\
& +\mu_{4}(y-\sqrt{-1} x)^{4}+\mu_{5}(z+\sqrt{-1} y)^{4}+\mu_{6}(z-\sqrt{-1} y)^{4}
\end{aligned}
$$

for some $\left(\mu_{1}, \mu_{2}, \mu_{3}, \mu_{4}, \mu_{5}, \mu_{6}\right) \in \mathbb{C}^{6}$. In particular, we obtain a system of linear equations

$$
\left\{\begin{array}{l}
4 \mu_{3}+4 \mu_{4}=1-\sqrt{-7} \\
4 \mu_{1}+4 \mu_{2}=1-\sqrt{-7} \\
4 \mu_{5}+4 \mu_{6}=1-\sqrt{-7} \\
\mu_{1}+\mu_{2}+\mu_{3}+\mu_{4}=1
\end{array}\right.
$$


which is inconsistent.

Thus, we see that $\Omega \cong \mathbb{Z}_{2} \times \mathbb{Z}_{2}$.

Lemma 4.20. The subgroup $\Omega$ is not contained in a subgroup of the group $G$ isomorphic to $\mathrm{A}_{4}$.

Proof. Suppose that $\Omega$ is contained in a subgroup of the group $G$ that is isomorphic to $\mathrm{A}_{4}$. Then

$$
\Omega=\left\{\left(\begin{array}{lll}
1 & 0 & 0 \\
0 & 1 & 0 \\
0 & 0 & 1
\end{array}\right),\left(\begin{array}{ccc}
1 & 0 & 0 \\
0 & -1 & 0 \\
0 & 0 & -1
\end{array}\right),\left(\begin{array}{ccc}
-1 & 0 & 0 \\
0 & -1 & 0 \\
0 & 0 & 1
\end{array}\right),\left(\begin{array}{ccc}
-1 & 0 & 0 \\
0 & 1 & 0 \\
0 & 0 & -1
\end{array}\right)\right\},
$$

which implies that the $G$-orbit of the line $L_{1}$ is the curve that is given by $x y z=0$, which is impossible by Lemma 4.18 .

By Lemma 4.20, we may assume that $\Omega$ is generated by $\psi(A B)$ and $\psi(C)$. Therefore

$$
\Omega=\left\{\left(\begin{array}{lll}
1 & 0 & 0 \\
0 & 1 & 0 \\
0 & 0 & 1
\end{array}\right),\left(\begin{array}{ccc}
0 & -1 & 0 \\
-1 & 0 & 0 \\
0 & 0 & -1
\end{array}\right),\left(\begin{array}{ccc}
0 & 1 & 0 \\
1 & 0 & 0 \\
0 & 0 & -1
\end{array}\right),\left(\begin{array}{ccc}
-1 & 0 & 0 \\
0 & -1 & 0 \\
0 & 0 & 1
\end{array}\right)\right\},
$$

and the equation of the hexagon $\sum_{i=1}^{6} L_{i}$ is $\left(x^{2}-z^{2}\right)\left(y^{2}-x^{2}\right)\left(z^{2}-y^{2}\right)=0$. Arguing as in the proof of Lemma 4.19, we obtain a contradiction.

The assertion of Theorem 4.8 is proved.

\section{Proof of Theorem 1.9}

Throughout this section we use the assumptions and notation of Section 3 , Suppose that Theorem 1.9 is false. Let us derive a contradiction.

Lemma 5.1. There is a $G$-invariant linear system $\mathcal{M}$ without fixed components on $\mathbb{P}^{3}$ such that $\mathbb{N} \mathbb{C S}\left(\mathbb{P}^{3}, \lambda \mathcal{M}\right) \neq \varnothing$ and $\mathbb{N} \mathbb{C S}\left(\mathbb{P}^{3}, \lambda^{\prime} \tau(\mathcal{M})\right) \neq \varnothing$, where $\lambda$ and $\lambda^{\prime}$ are positive rational numbers such that $\lambda \mathcal{M} \sim_{\mathbb{Q}} \lambda^{\prime} \tau(\mathcal{M}) \sim_{\mathbb{Q}} \mathcal{O}_{\mathbb{P}^{3}}(4)$.

Proof. The required assertion is well-known (see [10], [5, Theorem A.16], [5, Corollary A.22]).

The assertion of Lemma 5.1 is known as the Noether-Fano inequality.

Lemma 5.2. Either $\operatorname{mult}_{C_{6}}(\mathcal{M}) \leqslant 1 / \lambda$ or $\operatorname{mult}_{C_{6}}(\tau(\mathcal{M})) \leqslant 1 / \lambda^{\prime}$.

Proof. This is easy (cf. the proof of [5, Lemma B.15]).

Without loss of generality, we may assume that $\operatorname{mult}_{C_{6}}(\mathcal{M}) \leqslant 1 / \lambda$. Then $C_{6} \notin \mathbb{N} \mathbb{C S}\left(\mathbb{P}^{3}, \lambda \mathcal{M}\right)$. Note that the set $\mathbb{N L} \mathbb{C S}\left(\mathbb{P}^{3}, 2 \lambda \mathcal{M}\right)$ contains every center in $\mathbb{N} \mathbb{C S}\left(\mathbb{P}^{3}, \lambda \mathcal{M}\right)$ by Lemma 2.8. However the set $\mathbb{N L} \mathbb{C S}\left(\mathbb{P}^{3}, 2 \lambda \mathcal{M}\right)$ may be non-empty even if $\mathbb{N} \mathbb{C S}\left(\mathbb{P}^{3}, \lambda \mathcal{M}\right)=\varnothing$ (see Example 3.11).

Lemma 5.3. Let $\Lambda$ be a union of all curves contained in $\mathbb{N L} \mathbb{C S}\left(\mathbb{P}^{3}, 2 \lambda \mathcal{M}\right)$. Then $\operatorname{deg}(\Lambda) \leqslant 15$.

Proof. Let $M_{1}$ and $M_{2}$ be general surfaces in $\mathcal{M}$, and let $H$ be a sufficiently general hyperplane in $\mathbb{P}^{3}$. Then

$$
16 / \lambda^{2}=H \cdot M_{1} \cdot M_{2} \geqslant \sum_{P \in \Lambda \cap H} \operatorname{mult}_{P}\left(M_{1} \cdot M_{2}\right)>\operatorname{deg}(\Lambda) / \lambda^{2}
$$

by Theorem 2.5 , which implies that $\operatorname{deg}(\Lambda) \leqslant 15$. 
Lemma 5.4. The set $\mathbb{N} \mathbb{C}\left(\mathbb{P}^{3}, \lambda \mathcal{M}\right)$ does not contain any point in $\Sigma_{8} \sqcup C_{6}$.

Proof. Let $M_{1}$ and $M_{2}$ be general surfaces in $\mathcal{M}$, and let $Q$ be a general surface in $\mathcal{Q}$. Then

$$
32 / \lambda^{2}=Q \cdot M_{1} \cdot M_{2} \geqslant \sum_{O_{i} \in \Sigma_{8}} \operatorname{mult}_{O_{i}}\left(M_{1} \cdot M_{2}\right)=8 \operatorname{mult}_{O_{i}}\left(M_{1} \cdot M_{2}\right),
$$

which implies that $\mathbb{N} \mathbb{C S}\left(\mathbb{P}^{3}, \lambda \mathcal{M}\right)$ does not contain any point in $\Sigma_{8}$ by Theorem 2.7

Suppose that $\mathbb{N} \mathbb{C}\left(\mathbb{P}^{3}, \lambda \mathcal{M}\right)$ contains a point $P \in C_{6}$. Put $M_{1} \cdot M_{2}=\nu C_{6}+\Upsilon$, where $\mu$ is a non-negative integer, and $\Upsilon$ is an effective one-cycle such that $C_{6} \not \subset \operatorname{Supp}(\Upsilon)$. Then $\operatorname{mult}_{P}\left(\nu C_{14}+\Upsilon\right) \geqslant 4 / \lambda^{2}$ by Theorem[2.7. Let $\Theta$ be the $G$-orbit of the point $P$. Then $|\Theta| \geqslant 24$ by Lemma 2.15.

Let $S$ be a general cubic surface in $\mathbb{P}^{3}$ such that $C_{6} \subset S$. Then

$$
48 / \lambda^{2}-18 \nu=S \cdot \Upsilon \geqslant \sum_{O \in \Theta} \operatorname{mult}_{O}(\Upsilon)=|\Theta| \operatorname{mult}_{O}(\Upsilon)>24\left(4 / \lambda^{2}-\nu\right),
$$

because $C_{6}$ is a scheme-theoretic intersection of cubic surfaces in $\mathbb{P}^{3}$. Thus $\nu>8 / \lambda^{2}$. Let $H$ be a sufficiently general hyperplane in $\mathbb{P}^{3}$. Then

$$
16 / \lambda^{2}=H \cdot M_{1} \cdot M_{2}=\nu H \cdot C_{6}+H \cdot \Upsilon \geqslant \nu H \cdot C_{6}=6 \mu,
$$

which implies that $\nu \leqslant 8 /\left(3 \lambda^{2}\right)$, which is a contradiction, since $\nu>8 / \lambda^{2}$.

Lemma 5.5. The set $\mathbb{N} \mathbb{C S}\left(\mathbb{P}^{3}, \lambda \mathcal{M}\right)$ does not contain the curve $C_{14}$.

Proof. Suppose that $C_{14} \in \mathbb{N} \mathbb{C S}\left(\mathbb{P}^{3}, \lambda \mathcal{M}\right)$. Let us put $\mu=\operatorname{mult}_{C_{14}}(\mathcal{M})$ and $m=\operatorname{mult}_{O_{1}}(\mathcal{M})$. Then $\mu>1 / \lambda$ because $C_{14} \in \mathbb{N} \mathbb{C} S\left(\mathbb{P}^{3}, \lambda \mathcal{M}\right)$.

Let us find an upper bound for $\mu$. Let $M_{1}$ and $M_{2}$ be general surfaces in $\mathcal{M}$, and let $H$ be a sufficiently general hyperplane in $\mathbb{P}^{3}$. Then

$$
16 / \lambda^{2}=H \cdot M_{1} \cdot M_{2} \geqslant \sum_{P \in C_{14} \cap H} \operatorname{mult}_{C_{14}}\left(M_{1} \cdot M_{2}\right) \geqslant 14 \mu^{2},
$$

which implies that $\mu \leqslant \sqrt{8 / 7} / \lambda$. Thus, we have $1 / \lambda<\mu \leqslant \sqrt{8 / 7} / \lambda$.

Let us find a lower and an upper bound for $m$.

Let $\bar{C}_{14}$ and $\overline{\mathcal{M}}$ be the proper transforms of the curve $C_{14}$ and the linear system $\mathcal{M}$ on the threefold $U$, respectively, let $C$ be a general conic in $E_{1} \cong \mathbb{P}^{2}$ such that $\bar{C}_{14} \cap E_{1} \subset C$. Then $\left|\bar{C}_{14} \cap E_{1}\right|=3$ and the points of the set $\bar{C}_{14} \cap E_{1}$ are noncoplanar (see the proof of Lemma 2.11), which implies that the conic $C$ is not contained in the base locus of the linear system $\overline{\mathcal{M}}$. Hence

$$
2 m=C \cdot \overline{\mathcal{M}} \geqslant \sum_{P \in \bar{C}_{14} \cap E_{1}} \operatorname{mult}_{P}(\overline{\mathcal{M}}) \geqslant \sum_{P \in \bar{C}_{14} \cap E_{1}} \operatorname{mult}_{\bar{C}_{14}}(\overline{\mathcal{M}})=3 \mu,
$$

which implies that $m \geqslant 3 \mu / 2$. Thus, we see that $m>3 /(2 \lambda)$, since $\mu>1 / \lambda$.

Let us find an upper bound for $m$ (a trivial upper bound $m \leqslant 2 / \lambda$ follows by Lemma 5.4).

Let $\bar{M}_{1}$ and $\bar{M}_{2}$ be the proper transforms on $U$ of the surfaces $M_{1}$ and $M_{2}$, respectively, and let $\bar{Q}$ be the proper transform of a general surface in the linear system $\mathcal{Q}$. Then

$$
\begin{aligned}
32 / \lambda^{2}-8 m^{2}=\bar{Q} \cdot \bar{M}_{1} \cdot \bar{M}_{2} \geqslant \sum_{P \in \bar{C}_{14} \cap \bar{Q}} \operatorname{mult}_{\bar{C}_{14}}\left(\bar{M}_{1} \cdot \bar{M}_{2}\right)= \\
\quad=4 \operatorname{mult}_{\bar{C}_{14}}\left(\bar{M}_{1} \cdot \bar{M}_{2}\right) \geqslant 4 \mu^{2} \geqslant 4 / \lambda^{2},
\end{aligned}
$$


which implies that $m \leqslant \sqrt{7 / 2} / \lambda$. Thus, we have $3 /(2 \lambda)<m \leqslant \sqrt{7 / 2} / \lambda$.

Let $v: W \rightarrow U$ be the blow up of the smooth curve $\bar{C}_{14}$, let $F$ be the $v$-exceptional divisor, let $\hat{\mathcal{M}}$ and $\hat{\mathcal{D}}$ be the proper transforms of $\mathcal{M}$ and $\mathcal{D}$ (see Lemma 3.16) on $W$, respectively. Then

$$
\left\{\begin{array}{l}
\hat{\mathcal{M}} \sim_{\mathbb{Q}}(\pi \circ v)^{*}\left(\frac{4}{\lambda} H\right)-m \sum_{i=1}^{8} v^{*}\left(E_{i}\right)-\mu F, \\
\hat{\mathcal{D}} \sim_{\mathbb{Q}}(\pi \circ v)^{*}(5 H)-2 \sum_{i=1}^{8} v^{*}\left(E_{i}\right)-F .
\end{array}\right.
$$

Let $\hat{D}$ be a general surface in $\hat{\mathcal{D}}$. Then $\hat{D}$ is nef by Lemma 3.16 and (5.6).

Let $\hat{M}_{1}$ and $\hat{M}_{2}$ be the proper transforms on $W$ of the surfaces $M_{1}$ and $M_{2}$, respectively. Then

$$
80 / \lambda^{2}-2\left(17 \mu^{2}+4 m^{2}+56 \mu / \lambda-24 m \mu\right)=\hat{D} \cdot \hat{M}_{1} \cdot \hat{M}_{2} \geqslant 0
$$

because $\hat{D}$ is nef and $F^{3}=-12$. Put $\bar{\mu}=\mu \lambda$ and $\bar{m}=m \lambda$. One has

$$
17 \bar{\mu}^{2}+4 \bar{m}^{2}+56 \bar{\mu}-24 \bar{m} \bar{\mu} \geqslant 40, \quad 1<\bar{\mu} \leqslant \sqrt{\frac{8}{7}}, \quad \frac{3}{2}<\bar{m} \leqslant \sqrt{\frac{7}{2}},
$$

which easily leads to a contradiction.

Lemma 5.7. The set $\mathbb{N} \mathbb{C S}\left(\mathbb{P}^{3}, \lambda \mathcal{M}\right)$ does not contain any point in $C_{14}$.

Proof. Suppose that $\mathbb{N} \mathbb{C S}\left(\mathbb{P}^{3}, \lambda \mathcal{M}\right)$ contains a point $P \in C_{14}$. By Lemma 5.4 , we have $P \notin \Sigma_{8}$, which implies that $P$ is a smooth point of the curve $C_{14}$, by Lemma 3.13 .

Choose $M_{1}$ and $M_{2}$ to be general surfaces in the linear system $\mathcal{M}$. Put $M_{1} \cdot M_{2}=\mu C_{14}+\Upsilon+\Xi$, where $\mu$ is a non-negative integer, and $\Upsilon$ and $\Xi$ are effective one-cycles such that $C_{14} \not \subset \operatorname{Supp}(\Upsilon)$, every irreducible component of the curve $\operatorname{Supp}(\Upsilon)$ intersects the curve $C_{14}$ by a non-empty set, and none of the irreducible components of the curve $\operatorname{Supp}(\Xi)$ intersects $C_{14}$. Then $\operatorname{mult}_{P}\left(\mu C_{14}+\Upsilon\right) \geqslant 4 / \lambda^{2}$ by Theorem 2.7.

Let $H$ be a sufficiently general hyperplane in $\mathbb{P}^{3}$. Then

$$
16 / \lambda^{2}=H \cdot M_{1} \cdot M_{2}=\mu H \cdot C_{14}+H \cdot(\Upsilon+\Xi) \geqslant \mu H \cdot C_{14}=14 \mu,
$$

which implies that $\mu \leqslant 8 /\left(7 \lambda^{2}\right)$.

Let $\Theta$ be the $G$-orbit of the point $P$. Then $|\Theta| \geqslant 42$ by Lemma 2.15 .

Let $D$ be a general quintic surface in the linear system $\mathcal{D}$ (see Lemma 3.16 ). Then

$$
\begin{aligned}
80 / \lambda^{2}-70 \mu \geqslant 80 / \lambda^{2}-70 \mu-D \cdot \Xi=D \cdot \Upsilon & \geqslant \sum_{O \in \Theta} \operatorname{mult}_{O}(\Upsilon)= \\
& =|\Theta| \operatorname{mult}_{O}(\Upsilon)>42\left(4 / \lambda^{2}-\mu\right),
\end{aligned}
$$

which immediately leads to a contradiction.

Thus, the set $\mathbb{N L} \mathbb{C S}\left(\mathbb{P}^{3}, 2 \lambda \mathcal{M}\right)$ contains a center that is not contained in $C_{14} \sqcup C_{6}$.

Lemma 5.8. Suppose that $\mathbb{N L} \mathbb{C S}\left(\mathbb{P}^{3}, 2 \lambda \mathcal{M}\right)$ contains a curve $C$ different from both $C_{6}$ and $C_{14}$. Then $C_{14} \notin \mathbb{N L} \mathbb{C S}\left(\mathbb{P}^{3}, 2 \lambda \mathcal{M}\right)$, and if $C_{6} \in \mathbb{N L} \mathbb{C S}\left(\mathbb{P}^{3}, \lambda \mathcal{M}\right)$, then one has $C_{6} \cap C=\varnothing$ and $\operatorname{deg}(C) \leqslant 9$. 
Proof. Let $Z$ be a $G$-orbit of the curve $C$. If $C_{14} \in \mathbb{N L} \mathbb{C S}\left(\mathbb{P}^{3}, 2 \lambda \mathcal{M}\right)$, then $\operatorname{deg}(Z)+14=\operatorname{deg}(Z)+\operatorname{deg}\left(C_{14}\right) \leqslant 15$ by Lemma 5.3, which is a contradiction, because there are no $G$-invariant lines in $\mathbb{P}^{3}$.

Suppose that $C_{6} \in \mathbb{N L} \mathbb{C S}\left(\mathbb{P}^{3}, \lambda \mathcal{M}\right)$. Then $\operatorname{deg}(C) \leqslant \operatorname{deg}(Z) \leqslant 9$ by Lemma 5.3 . Thus, to complete the proof, we must show that $C_{6} \cap Z=\varnothing$.

Suppose that $C_{6} \cap Z \neq \varnothing$. Then $\left|C_{6} \cap Z\right| \geqslant 24$ by Lemma 2.15, Let $\bar{Z}$ be a proper transform of the curve $Z$ on the threefold $V$ (see (3.9)), and let $\bar{S}$ be a proper transform on $V$ of a general cubic surface in $\mathbb{P}^{3}$ that passes through the curve $C_{6}$. Then

$$
3 \geqslant 3 \operatorname{deg}(Z)-\left|C_{6} \cap Z\right| \geqslant \bar{S} \cdot \bar{Z}=\operatorname{deg}(\beta(\bar{Z})),
$$

which implies that $\bar{Z}$ is contracted by $\beta$ by Lemma 3.7, since $\beta(\bar{Z})$ is $G$-invariant.

The only curves contracted by the morphism $\beta$ are proper transforms of the lines in $\mathbb{P}^{3}$ that are trisecants of the curve $C_{6}$. Then $\beta(\bar{Z}) \subset C_{6}$, the subset $\beta(\bar{Z})$ is $G$-invariant and $|\beta(\bar{Z})| \leqslant 9$, which is impossible by Lemma 2.15.

It follows from Lemmas $5.4,5.5$ and 5.7 that the set $\mathbb{N L} \mathbb{C S}\left(\mathbb{P}^{3}, 2 \lambda \mathcal{M}\right)$ contains an irreducible subvariety that is not contained in $C_{6} \cup C_{14}$. In fact, we can say a little bit more than this.

Lemma 5.9. There are $\mu \in \mathbb{Q}$ and $S \in \mathbb{L} \mathbb{C}\left(\mathbb{P}^{3}, \mu \mathcal{M}\right)$ such that

- the inequalities $0<\mu<2 \lambda$ holds,

- the log pair $\left(\mathbb{P}^{3}, \mu \mathcal{M}\right)$ is log canonical along $S$,

- the subvariety $S$ is a minimal center in $\mathbb{L} \mathbb{C} S\left(\mathbb{P}^{3}, \mu \mathcal{M}\right)$,

- the subvariety $S$ is not a point of the set $\Sigma_{8}$,

- the subvariety $S$ is neither the curve $C_{6}$ nor the curve $C_{14}$,

- exactly one of the following six cases is possible:

(A) the log pair $\left(\mathbb{P}^{3}, \mu \mathcal{M}\right)$ has log canonical singularities,

(B) $\operatorname{NCS}\left(\mathbb{P}^{3}, \mu \mathcal{M}\right)=C_{14}$ and $S$ is a point such that $S \notin C_{14}$,

(C) $\operatorname{NCS}\left(\mathbb{P}^{3}, \mu \mathcal{M}\right)=\Sigma_{8}$ and $S \cap \Sigma_{8}=\varnothing$,

(D) $\operatorname{NCS}\left(\mathbb{P}^{3}, \mu \mathcal{M}\right)=C_{6}$ and $S$ is a curve such that $S \cap C_{6}=\varnothing$ and $\operatorname{deg}(S) \leqslant 9$

(E) $\operatorname{NCS}\left(\mathbb{P}^{3}, \mu \mathcal{M}\right)=C_{6}$ and $S$ is a point such that $S \notin \Sigma_{8}$,

(F) $\operatorname{NCS}\left(\mathbb{P}^{3}, \mu \mathcal{M}\right)=C_{6} \cup \Sigma_{8}$ and $S$ is a curve such that one has $S \cap\left(C_{6} \cup \Sigma_{8}\right)=\varnothing$ and $\operatorname{deg}(S) \leqslant 9$,

(G) $\operatorname{NCS}\left(\mathbb{P}^{3}, \mu \mathcal{M}\right)=C_{6} \cup \Sigma_{8}$ and $S$ is a point such that $S \notin C_{6} \cup \Sigma_{8}$.

Proof. Let us show how to find $\mu$ and $S$ in several steps. Put

$$
\mu_{1}=\sup \left\{\epsilon \in \mathbb{Q} \mid \text { the log pair }\left(\mathbb{P}^{3}, \epsilon \mathcal{M}\right) \text { is log canonical }\right\},
$$

and let $S_{1}$ be a minimal center in $\mathbb{L} \mathbb{C}\left(\mathbb{P}^{3}, \mu_{1} \mathcal{M}\right)$. Then $\mu_{1}<2 \lambda$.

If $S_{1}$ is a curve, then $S_{1} \cap \Sigma_{8}=\varnothing$ by Lemmas 3.14 and 5.3. since $S_{1}$ is smooth by Theorem 2.10. If $S_{1} \cap \Sigma_{8}=\varnothing$ and $S_{1} \neq C_{6}$, then we have the case (A) by putting $\mu=\mu_{1}$ and $S=S_{1}$. Thus, to complete the proof, we may assume that either $S_{1} \cap \Sigma_{8} \neq \varnothing$ or $S_{1}=C_{6}$.

Let us consider the mutually excluding cases $S_{1} \cap \Sigma_{8} \neq \varnothing$ and $S_{1}=C_{6}$ separately.

Suppose that $S_{1}=C_{6}$. Put

$$
\mu_{2}=\sup \left\{\epsilon \in \mathbb{Q} \mid \text { the } \log \text { pair }\left(\mathbb{P}^{3}, \epsilon \mathcal{M}\right) \text { is } \log \text { canonical outside } C_{6}\right\}<2 \lambda,
$$

let $T_{2}$ be a center in the set $\mathbb{L} \mathbb{C S}\left(\mathbb{P}^{3}, \mu_{2} \mathcal{M}\right)$ such that $T_{2} \neq C_{6}$, and let $S_{2}$ be a minimal center in the set $\mathbb{L} \mathbb{C S}\left(\mathbb{P}^{3}, \mu_{2} \mathcal{M}\right)$ such that $S_{2} \subseteq T_{2}$. If $T_{2}$ is a curve, 
then $\operatorname{deg}\left(T_{2}\right) \leqslant 9$ and $T_{2} \cap \Sigma_{8}=T_{2} \cap C_{6}=\varnothing$ by Lemmas 3.14, 5.3 and 5.8, since $C_{14} \cap C_{6}=\varnothing$ by Lemma 3.13 and $C_{6} \in \mathbb{N L} \mathbb{C S}\left(\mathbb{P}^{3}, 2 \lambda \mathcal{M}\right)$.

If $T_{2}$ is a curve and $S_{2}=T_{2}$, then we have the case (D) by putting $\mu=\mu_{2}$ and $S=S_{2}$. If $T_{2}$ is a curve and $S_{2}$ is a point, then we have the case (E) by putting $\mu=\mu_{2}$ and $S=S_{2}$. If $T_{2}=S_{2}$ is a point not in $\Sigma_{8}$, then we have the case (E) by putting $\mu=\mu_{2}$ and $S=S_{2}$. Thus, to finish the case $S_{1}=C_{6}$, we may assume that $S_{2}=T_{2}$ is a point in $\Sigma_{8}$. Put

$\mu_{3}=\sup \left\{\epsilon \in \mathbb{Q} \mid\right.$ the log pair $\left(\mathbb{P}^{3}, \epsilon \mathcal{M}\right)$ is log canonical outside $\left.C_{6} \cup \Sigma_{8}\right\}<2 \lambda$,

let $T_{3}$ be a center in the set $\mathbb{L} \mathbb{C S}\left(\mathbb{P}^{3}, \mu_{3} \mathcal{M}\right)$ such that $T_{3} \not \subset C_{6} \cup \Sigma_{8}$, and let $S_{3}$ be a minimal center in the set $\mathbb{L} \mathbb{C S}\left(\mathbb{P}^{3}, \mu_{3} \mathcal{M}\right)$ such that $S_{3} \subseteq T_{3}$. If $T_{3}$ is a curve, then $\operatorname{deg}\left(T_{3}\right) \leqslant 9$ and $T_{3} \cap \Sigma_{8}=T_{3} \cap C_{6}=\varnothing$ by Lemmas [3.14, 5.3 and [5.8, since $C_{14} \cap C_{6}=\varnothing$ by Lemma 3.13 and $C_{6} \in \mathbb{N L} \mathbb{C S}\left(\mathbb{P}^{3}, 2 \lambda \mathcal{M}\right)$.

If $T_{3}$ is a curve and $S_{3}=T_{3}$, then we have the case $(\mathbf{F})$ by putting $\mu=\mu_{2}$ and $S=S_{2}$. If $T_{2}$ is a curve and $S_{2}$ is a point, then we have the case $(\mathbf{G})$ by putting $\mu=\mu_{2}$ and $S=S_{2}$. If $T_{2}=S_{2}$ is a point, then we have the case (G) by putting $\mu=\mu_{2}$ and $S=S_{2}$. Therefore, the case $S_{1}=C_{6}$ is done, and we may assume that $S_{1} \cap \Sigma_{8} \neq \varnothing$, which implies that the subvariety $S_{1}$ is a point in $\Sigma_{8}$ by Theorem 2.10 and Lemmas 3.14 and 5.3, Put

$\mu_{2}^{\prime}=\sup \left\{\epsilon \in \mathbb{Q} \mid\right.$ the $\log$ pair $\left(\mathbb{P}^{3}, \epsilon \mathcal{M}\right)$ is log canonical outside $\left.\Sigma_{8}\right\}<2 \lambda$,

let $T_{2}^{\prime}$ be a center in the set $\mathbb{L} \mathbb{C} S\left(\mathbb{P}^{3}, \mu_{2}^{\prime} \mathcal{M}\right)$ such that $T_{2}^{\prime} \not \subset \Sigma_{8}$, and let $S_{2}^{\prime}$ be a minimal center in the set $\mathbb{L} \mathbb{C S}\left(\mathbb{P}^{3}, \mu_{2}^{\prime} \mathcal{M}\right)$ such that $S_{2}^{\prime} \subseteq T_{2}^{\prime}$. Note that if $S_{2}^{\prime}$ is a point in $\Sigma_{8}$, then $T_{2}^{\prime} \neq S_{2}^{\prime}$ which implies that $T_{2}^{\prime}$ must be the curve $C_{14}$ by Lemmas 3.14 and 5.3 .

If $T_{2}^{\prime}$ is a point, then $S_{2}^{\prime}=T_{2}^{\prime}$, and we have the case $(\mathbf{C})$ by putting $\mu=\mu_{2}^{\prime}$ and $S=S_{2}^{\prime}$. So, to complete the proof, we may assume that $T_{2}^{\prime}$ is an irreducible curve.

If $S_{2}^{\prime}=T_{2}^{\prime}$, then $S_{2}^{\prime}$ is a smooth curve such that $\operatorname{deg}\left(S_{2}^{\prime}\right) \leqslant 15$ by Theorem 2.10 and Lemma 5.3. which immediately implies that $T_{2}^{\prime} \cap \Sigma_{8}=\varnothing$ by Lemma 3.14. If $S_{2}^{\prime}=T_{2}^{\prime} \neq C_{6}$, then we have the case $(\mathbf{C})$ by putting $\mu=\mu_{2}^{\prime}$ and $S=S_{2}^{\prime}$. If $S_{2}^{\prime}$ is a point not in $\Sigma_{8}$, then we have the case $(\mathbf{C})$ by putting $\mu=\mu_{2}^{\prime}$ and $S=S_{2}^{\prime}$. Hence, to complete the proof, we may assume that either $S_{2}^{\prime}$ is a point of the set $\Sigma_{8}$ and $T_{2}^{\prime}=C_{14}$, or we have $S_{2}^{\prime}=T_{2}^{\prime}=C_{6}$. Let us consider these cases separately.

Suppose that $S_{2}^{\prime}$ is a point of the set $\Sigma_{8}$ and $T_{2}^{\prime}=C_{14}$. Put

$$
\mu_{3}^{\prime}=\sup \left\{\epsilon \in \mathbb{Q} \mid \text { the } \log \text { pair }\left(\mathbb{P}^{3}, \epsilon \mathcal{M}\right) \text { is } \log \text { canonical outside } C_{14}\right\}<2 \lambda,
$$

let $T_{3}^{\prime}$ be a center in the set $\mathbb{L} \mathbb{C S}\left(\mathbb{P}^{3}, \mu_{3}^{\prime} \mathcal{M}\right)$ such that $T_{3}^{\prime} \not \subset C_{14}$. Then $T_{3}$ is a point by Lemma 5.8, which implies that we have the case (B) by putting $\mu=\mu_{3}^{\prime}$ and $S=T_{3}^{\prime}$.

To complete the proof, we may assume that $S_{2}^{\prime}=T_{2}^{\prime}=C_{6}$. Put $\mu_{3}^{\prime \prime}=\sup \left\{\epsilon \in \mathbb{Q} \mid\right.$ the log pair $\left(\mathbb{P}^{3}, \epsilon \mathcal{M}\right)$ is log canonical outside $\left.C_{6} \cup \Sigma_{8}\right\}<2 \lambda$, let $T_{3}^{\prime}$ be a center in the set $\mathbb{L} \mathbb{C}\left(\mathbb{P}^{3}, \mu_{3}^{\prime \prime} \mathcal{M}\right)$ such that $T_{3}^{\prime \prime} \not \subset C_{6} \cap \Sigma_{8}$, and let $S_{3}^{\prime \prime}$ be a minimal center in the set $\mathbb{L} \mathbb{C} S\left(\mathbb{P}^{3}, \mu_{3}^{\prime \prime} \mathcal{M}\right)$ such that $S_{3}^{\prime \prime} \subseteq T_{3}^{\prime \prime}$. Note that $C_{14} \notin \mathbb{L} \mathbb{C} S\left(\mathbb{P}^{3}, 2 \lambda \mathcal{M}\right)$ by Lemma 5.8.

If $T_{3}^{\prime \prime}$ is a point, then $S_{3}^{\prime \prime}=T_{3}^{\prime \prime}$, and we have the case $(\mathbf{G})$ by putting $\mu=\mu_{3}^{\prime \prime}$ and $S=S_{3}^{\prime \prime}$. Thus, we may assume that $T_{3}^{\prime \prime}$ is a curve. Then one has $\operatorname{deg}\left(T_{3}^{\prime \prime}\right) \leqslant 9$ and $T_{3}^{\prime \prime} \cap C_{6}=T_{3}^{\prime \prime} \cap \Sigma_{8}=\varnothing$ by Lemmas 3.14 , 5.3 and 5.8 , since $C_{6} \in \mathbb{L} \mathbb{C S}\left(\mathbb{P}^{3}, 2 \lambda \mathcal{M}\right) \not \supset C_{14}$. Finally, we put $\mu=\mu_{3}^{\prime \prime}$ and $S=S_{3}^{\prime \prime}$. Then we 
have the case $(\mathbf{G})$ if the center $S_{3}^{\prime \prime}$ is a point, and we have the case $(\mathbf{F})$ if the center $S_{3}^{\prime \prime}$ is a curve, which completes the proof.

Let $\epsilon$ be any rational number such that $\mu<\epsilon \mu<2 \lambda$. Then it follows from Lemma 2.13 that there is a $G$-invariant linear system $\mathcal{B}$ on $\mathbb{P}^{3}$ such that $\mathcal{B}$ does not have fixed components, and there are positive rational numbers $\epsilon_{1}$ and $\epsilon_{2}$ such that $1 \geqslant \epsilon_{1} \gg \epsilon_{2} \geqslant 0$ and

$$
\mathbb{L} \mathbb{C} S\left(\mathbb{P}^{3}, \epsilon_{1} \mu \mathcal{M}+\epsilon_{2} \mathcal{B}\right)=\left(\bigsqcup_{g \in G}\{g(S)\}\right) \bigsqcup \mathbb{N L} \mathbb{C} S\left(\mathbb{P}^{3}, \mu \mathcal{M}\right),
$$

the log pair $\left(\mathbb{P}^{3}, \epsilon_{1} \mu \mathcal{M}+\epsilon_{2} \mathcal{B}\right)$ is log canonical at every point of $g(Z)$ for every $g \in G$, and $\epsilon_{1} \mu \mathcal{M}+\epsilon_{2} \mathcal{B} \sim \mathbb{Q} \epsilon \mu \mathcal{M}$. Let $Z$ be the $G$-orbit of the subvariety $S$. Then one of the following cases is possible:

(A) $\operatorname{LCS}\left(\mathbb{P}^{3}, \epsilon_{1} \mu \mathcal{M}+\epsilon_{2} \mathcal{B}\right)=Z$,

(B) $\operatorname{LCS}\left(\mathbb{P}^{3}, \epsilon_{1} \mu \mathcal{M}+\epsilon_{2} \mathcal{B}\right)=Z \sqcup C_{14}$ and $Z$ is finite set,

(C) $\operatorname{LCS}\left(\mathbb{P}^{3}, \epsilon_{1} \mu \mathcal{M}+\epsilon_{2} \mathcal{B}\right)=Z \sqcup \Sigma_{8}$,

(D) $\operatorname{LCS}\left(\mathbb{P}^{3}, \epsilon_{1} \mu \mathcal{M}+\epsilon_{2} \mathcal{B}\right)=Z \sqcup C_{6}$ and $Z$ is a curve such that $\operatorname{deg}(Z) \leqslant 9$,

(E) $\operatorname{LCS}\left(\mathbb{P}^{3}, \epsilon_{1} \mu \mathcal{M}+\epsilon_{2} \mathcal{B}\right)=Z \sqcup C_{6}$ and $Z$ is a finite set such that $Z \neq \Sigma_{8}$,

(F) $\operatorname{LCS}\left(\mathbb{P}^{3}, \epsilon_{1} \mu \mathcal{M}+\epsilon_{2} \mathcal{B}\right)=Z \sqcup C_{6} \sqcup \Sigma_{8}$ and $Z$ is a curve such that $\operatorname{deg}(Z) \leqslant 9$,

(G) $\operatorname{LCS}\left(\mathbb{P}^{3}, \epsilon_{1} \mu \mathcal{M}+\epsilon_{2} \mathcal{B}\right)=Z \sqcup C_{6} \sqcup \Sigma_{8}$ and $Z$ is a finite set.

Put $D=\epsilon_{1} \mu \mathcal{M}+\epsilon_{2} \mathcal{B}$. Let $\mathcal{L}$ be the union of all connected components of the log canonical singularity subscheme $\mathcal{L}\left(\mathbb{P}^{3}, D\right)$ whose supports contains no components of the subvariety $Z$. Then

$$
\operatorname{Supp}(\mathcal{L})=\left\{\begin{array}{l}
\varnothing \text { in the case }(\mathbf{A}), \\
C_{14} \text { in the case }(\mathbf{B}), \\
\Sigma_{8} \text { in the case }(\mathbf{C}), \\
C_{6} \text { in the cases }(\mathbf{D}) \text { and }(\mathbf{E}), \\
C_{6} \sqcup \Sigma_{8} \text { in the cases }(\mathbf{F}) \text { and }(\mathbf{G}),
\end{array}\right.
$$

Let $\mathcal{I}\left(\mathbb{P}^{3}, D\right)$ be the multiplier ideal sheaf of the log pair $\left(\mathbb{P}^{3}, D\right)$. Then

$$
h^{0}\left(\mathcal{O}_{\mathcal{L}} \otimes \mathcal{O}_{\mathbb{P}^{3}}(4)\right)+h^{0}\left(\mathcal{O}_{Z} \otimes \mathcal{O}_{\mathbb{P}^{3}}(4)\right)=35-h^{0}\left(\mathcal{O}_{\mathbb{P}^{3}}(4) \otimes \mathcal{I}\left(\mathbb{P}^{3}, D\right)\right) \leqslant 35
$$

by Theorem 2.2 .

Corollary 5.11. Suppose that $Z$ is a finite set. Then $Z$ contains at most 35 points, and $Z$ imposes independent linear conditions on quartic surfaces in $\mathbb{P}^{3}$.

Lemma 5.12. Suppose that $S$ is a point. Then $|Z| \neq 24$.

Proof. Suppose that $|Z|=24$. Then $Z=\Sigma_{24}$ by Lemma 3.2, and there is a hypersurface $F$ of degree 4 in $\mathbb{P}^{3}$ such that $Z \backslash S \subset F$ and $S \not \subset F$ by Corollary 5.11. Thus, there is a unique point $P \in C_{6}$ such that $P \neq S$ and $\left.F\right|_{C_{6}}=P+\left.Z \backslash S \sim F_{4}\right|_{C_{6}}=Z$, which implies that $P \sim S$ on $C_{6}$, which is impossible, since $C_{6}$ is a smooth curve of genus 3 .

Lemma 5.13. The subvariety $S$ is a curve.

Proof. Suppose that $S$ is a point. By Lemmas 3.2 and 5.12 and Corollary 5.11, we have $|Z|=28$, because $Z \neq \Sigma_{8}$. Without loss of generality, we may assume that $Z=\Sigma_{28}$.

Let $M_{1}$ and $M_{2}$ be general surfaces in $\mathcal{M}$. Put $M_{1} \cdot M_{1}=\Xi+\Lambda$, where $\Xi$ and $\Lambda$ are effective cycles such that $\operatorname{Supp}(\Xi) \cap \operatorname{Supp}(\Lambda)$ consists of finitely many points, 
and $\operatorname{Supp}(\Xi) \supset \Sigma_{28} \not \subset \operatorname{Supp}(\Lambda)$. If $S \in \mathbb{N} \mathbb{C S}\left(\mathbb{P}^{3}, \lambda \mathcal{M}\right)$, then $\operatorname{mult}_{S}(\Xi)>4 / \lambda^{2}$ by Theorem 2.7.

Let $R$ be a general surface in $\mathcal{R}$ (see Lemma 3.19). Then $\operatorname{Supp}(\Xi) \cap R$ consists of at most finitely many points by Lemma 3.19 and Corollary 5.11. Thus, we must have

$$
64 / \lambda^{2} \geqslant 64 / \lambda^{2}-R \cdot \Lambda=R \cdot \Xi \geqslant \sum_{P \in \Sigma_{28}} \operatorname{mult}_{P}(\Xi)=28 \operatorname{mult}_{S}(\Xi),
$$

which implies that $\operatorname{mult}_{S}(\Xi) \leqslant 16 /\left(7 \lambda^{2}\right)$. So $g(S) \notin \mathbb{N} \mathbb{C S}\left(\mathbb{P}^{3}, \lambda \mathcal{M}\right)$ for every $g \in G$.

Note that $\Sigma_{28} \not \subset C_{6} \sqcup C_{14}$ by Lemma 2.15, since $C_{6}$ is smooth and $\operatorname{Sing}\left(C_{14}\right)=\Sigma_{8}$.

It follows from Lemmas [5.4, 5.5 and 5.7 that the set $\mathbb{N L} \mathbb{C S}\left(\mathbb{P}^{3}, 2 \lambda \mathcal{M}\right)$ contains an irreducible subvariety that is not contained in $C_{6} \cup C_{14} \cup \Sigma_{28}$. Put

$$
\bar{\mu}=\sup \left\{\epsilon \in \mathbb{Q} \mid \text { the } \log \text { pair }\left(\mathbb{P}^{3}, \epsilon \mathcal{M}\right) \text { is log canonical outside } C_{6} \cup C_{14} \cup \Sigma_{28}\right\} .
$$

The set $\mathbb{L} \mathbb{C}\left(\mathbb{P}^{3}, \bar{\mu} \mathcal{M}\right)$ contains every point in $\Sigma_{28}$. If $\bar{\mu}>\mu$, then $\mathbb{N L} \mathbb{C S}\left(\mathbb{P}^{3}, \bar{\mu} \mathcal{M}\right)$ also contains every points in $\Sigma_{28}$. It follows from Lemma 2.8 that $\bar{\mu}<2 \lambda$. Note that $\bar{\mu} \geqslant \mu$.

Let $\Omega$ be a center $\mathbb{L} \mathbb{C S}\left(\mathbb{P}^{3}, \bar{\mu} \mathcal{M}\right)$ such that $\Omega \not \subset C_{6} \cup C_{14} \cup \Sigma_{28}$. Note that $\Omega$ does exist. Let us choose a center $\Gamma \in \mathbb{L} \mathbb{C S}\left(\mathbb{P}^{3}, \bar{\mu} \mathcal{M}\right)$ in the following way:

- if $\Omega$ is a point, then we put $\Gamma=\Omega$,

- if $\Omega$ is a curve that is a minimal center in $\mathbb{L} \mathbb{C S}\left(\mathbb{P}^{3}, \bar{\mu} \mathcal{M}\right)$, then we put $\Gamma=\Omega$,

- if $\Omega$ is a curve that is not a minimal center in $\mathbb{L} \mathbb{C}\left(\mathbb{P}^{3}, \bar{\mu} \mathcal{M}\right)$, then let $\Gamma$ be a point in $\Omega$ that is also a center in $\mathbb{L} \mathbb{C S}\left(\mathbb{P}^{3}, \bar{\mu} \mathcal{M}\right)$.

Let $\Delta$ be a $G$-orbit of the center $\Gamma$. Then $\Delta \cap \Sigma_{8}=\Delta \cap \Sigma_{28}=\varnothing$ by Lemmas 3.14, 3.17, 5.8, 5.3.

Let $\bar{\epsilon}$ be a rational number such that $\bar{\mu}<\bar{\epsilon} \bar{\mu}<2 \lambda$. Arguing as in the proof of Lemma 2.13, we obtain a $G$-invariant linear system $\mathcal{B}^{\prime}$ on $\mathbb{P}^{3}$ such that $\mathcal{B}^{\prime}$ does not have fixed components. Moreover, we can choose positive rational numbers $\bar{\epsilon}_{1}$ and $\bar{\epsilon}_{2}$ such that $1 \geqslant \bar{\epsilon}_{1} \gg \bar{\epsilon}_{2} \geqslant 0$ and

$$
\mathbb{L} \mathbb{C S}\left(\mathbb{P}^{3}, \bar{\epsilon}_{1} \bar{\mu} \mathcal{M}+\bar{\epsilon}_{2} \mathcal{B}^{\prime}\right)=\left(\bigsqcup_{g \in G}\{g(\Gamma)\}\right) \bigsqcup\left(\bigsqcup_{P \in \Sigma_{28}}\{P\}\right) \bigsqcup \mathbb{N L} \mathbb{C S}\left(\mathbb{P}^{3}, \bar{\mu} \mathcal{M}\right)
$$

if $\bar{\mu}=\mu$, or

$$
\mathbb{L} \mathbb{C S}\left(\mathbb{P}^{3}, \bar{\epsilon}_{1} \bar{\mu} \mathcal{M}+\bar{\epsilon}_{2} \mathcal{B}^{\prime}\right)=\left(\bigsqcup_{g \in G}\{g(\Gamma)\}\right) \bigsqcup \mathbb{N L} \mathbb{C S}\left(\mathbb{P}^{3}, \bar{\mu} \mathcal{M}\right)
$$

if $\bar{\mu}>\mu$.

Put $\bar{D}=\bar{\epsilon}_{1} \bar{\mu} \mathcal{M}+\bar{\epsilon}_{2} \mathcal{B}^{\prime}$. Then $\Gamma$ is a connected component of the subscheme $\mathcal{L}\left(\mathbb{P}^{3}, \bar{D}\right)$. Let $\overline{\mathcal{L}}$ be the union of all connected components of the subscheme $\mathcal{L}\left(\mathbb{P}^{3}, \bar{D}\right)$ whose supports contain no components of the subvariety $\Delta$. Then $h^{0}\left(\mathcal{O}_{\overline{\mathcal{L}}} \otimes \mathcal{O}_{\mathbb{P}^{3}}(4)\right) \geqslant 28$, since $\Sigma_{28} \subseteq \operatorname{Supp}(\overline{\mathcal{L}})$.

Let $\mathcal{I}\left(\mathbb{P}^{3}, \bar{D}\right)$ be the multiplier ideal sheaf of the log pair $\left(\mathbb{P}^{3}, \bar{D}\right)$. Then

$$
h^{0}\left(\mathcal{O}_{\Delta} \otimes \mathcal{O}_{\mathbb{P}^{3}}(4)\right)=35-h^{0}\left(\mathcal{O}_{\mathbb{P}^{3}}(4) \otimes \mathcal{I}\left(\mathbb{P}^{3}, \bar{D}\right)\right)-h^{0}\left(\mathcal{O}_{\overline{\mathcal{L}}} \otimes \mathcal{O}_{\mathbb{P}^{3}}(4)\right) \leqslant 7
$$

by Theorem 2.2, which implies that $\Gamma$ is not a point by Lemma 3.2. We see that $\Delta$ is a curve. By Theorem 2.10, the curve $\Delta$ is a smooth curve in $\mathbb{P}^{3}$ of degree $d$ and genus $g \leqslant 2 d$. Then

$$
\frac{\operatorname{deg}(\Delta)}{\operatorname{deg}(\Gamma)}(2 d+1) \leqslant \frac{\operatorname{deg}(\Delta)}{\operatorname{deg}(\Gamma)}(4 d-g+1) \leqslant 7
$$


which gives $d \leqslant 3$. Thus, we have $\Delta \neq \Gamma$, so that $\operatorname{deg}(\Delta) \geqslant 7 \operatorname{deg}(\Gamma)$ by Corollary B.2. We have

$$
21 \leqslant 7(2 d+1) \leqslant(2 d+1) \frac{\operatorname{deg}(\Delta)}{\operatorname{deg}(\Gamma)}(2 d+1) \leqslant \frac{\operatorname{deg}(\Delta)}{\operatorname{deg}(\Gamma)}(4 d-g+1) \leqslant 7,
$$

which is a contradiction.

By Theorem 2.10, the curve $S$ is a smooth curve of degree $d$ and genus $g$ such that $g \leqslant 2 d$, which implies, in particular, that the case $(\mathbf{B})$ is not possible by Lemma 5.8 .

Let $G_{S}$ be the stabilizer subgroup in $G$ of the subvariety $S$. Put $p=h^{0}\left(\mathcal{L} \otimes \mathcal{O}_{\mathbb{P}^{3}}(4)\right)$, put $q=h^{0}\left(\mathcal{O}_{\mathbb{P}^{3}}(4) \otimes \mathcal{I}\left(\mathbb{P}^{3}, D\right)\right)$, and let $r$ be the number of irreducible components of the curve $Z$. Then

$$
r(4 d-g+1)=35-q-p
$$

by (5.10), the Riemann-Roch theorem and Remark 2.12 .

Lemma 5.15. The equality $r=1$ holds.

Proof. Suppose that $r \neq 1$. Then $r \geqslant 7$ by Corollary B.2, which implies that

$$
4 d-g+1=\frac{35-q-p}{r} \leqslant 5
$$

by (5.14). But $g \leqslant 2 d$. Then $4 d-g+1 \leqslant 5$, which implies that $g=p=q=0$, $d=1$ and $r=7$.

The induced action of the group $G_{S} \cong \mathrm{S}_{4}$ on the line $S$ is faithful, which implies that $S \subset F_{4}$, since $G_{S}$-invariant subsets in $S$ have at least 6 points. Then $Z \subset F_{4}$, which contradicts $q=0$.

Therefore, we see that $Z=S$.

Remark 5.16. Let $I$ be the trivial representation of the group G. By Lemma B.8 we have

$$
H^{0}\left(\mathcal{O}_{\mathbb{P}^{3}}(4)\right) \cong I \oplus W_{6} \oplus W_{6} \oplus W_{7} \oplus W_{7} \oplus W_{8},
$$

where $W_{i}$ is an irreducible representation of the group $G \cong \mathrm{PSL}_{2}\left(\mathbb{F}_{7}\right)$ of dimension $i$. Then $p+q \notin\{2,3,4,5,10,11,17\}$, because $p$ is divisible by 8 . If $S \subset F_{4}$, then $p+q \notin\{0,2,3,4,5,6,10,11,12,17\}$.

Note that there is a natural faithful action of the group $G$ on the curve $S$.

Lemma 5.17. We have $g \in\{3,8,10,15,17,22,24,29\}$.

Proof. This follows from Lemmas 2.16 and 3.2 , since $g \leqslant 2 d \leqslant 30$ by Lemma 5.3 ,

Lemma 5.18. Suppose that $d \neq 6$ and $d \neq 12$. Then $S \subset F_{4}$.

Proof. Suppose that $S \not \subset F_{4}$. Then $F_{4} \cap S$ is union of some $G$-orbits $\Lambda_{1}, \ldots, \Lambda_{s}$. Thus $\sum_{i=1}^{s} n_{i}\left|\Lambda_{i}\right|=4 d$ for some positive integers $n_{1}, n_{2}, \ldots, n_{s}$. Using Lemma 2.16, we obtain that $d=14, s=1, n_{1}=1$ and $\left|\Lambda_{1}\right|=56$. Then $g=22+q+p \leqslant 2 d=28$, which implies that $g=22$ by Lemma 5.17 and Remark 5.16, By Lemma 2.16, we have $\left|\Lambda_{1}\right| \neq 56$, which is a contradiction.

Note that $d \in\{6,7,8,9\}$ in the cases $(\mathbf{D}),(\mathbf{E}),(\mathbf{F}),(\mathbf{G})$.

Lemma 5.19. The equality $d=6$ is impossible. 
Proof. Suppose that $d=6$. Then it follows from Lemma 5.17 that

$$
3 \leqslant g=q+p-10 \leqslant 2 d=12,
$$

which implies that $g \in\{3,8,10\}$. But $g \leqslant 4$ by Theorem 2.14. Hence $S=C_{6}$ by Lemma 3.7, which is a contradiction, because $S \neq C_{6}$ by the choice of the center $S \in \mathbb{L} \mathbb{C} S\left(\mathbb{P}^{3}, \mu \mathcal{M}\right)$.

Lemma 5.20. The equality $d=7$ is impossible.

Proof. Suppose that $d=7$. Then $g \leqslant 7$ by Theorem 2.14 and $g=3$ by Lemma 5.17 .

By Lemma 5.18, we see that $S \subset F_{4}$. Arguing as in the proof of Lemma 5.17, we see that the curve $S$ is contained in the intersection $F_{4} \cap F_{6} \cap F_{14}$, which is impossible by Lemma 3.6.

Lemma 5.21. The equality $d=8$ is impossible.

Proof. Suppose that $d=8$. Then $g \leqslant 9$ by Theorem 2.14 and $g \in\{3,8\}$ by Lemma 5.17. By Lemma 5.18, we see that $S \subset F_{4}$. Arguing as in the proof of Lemma 5.17, we see that the curve $S$ is contained in $F_{4} \cap F_{6} \cap F_{8}^{\prime}$ if $g=8$. Thus $g=3$ by Lemma 3.6. Then $\left.\mathcal{O}_{\mathbb{P}^{3}}(1)\right|_{S} \sim 2 K_{S}$ by Theorem 2.19, which is impossible, since the vector space $H^{0}\left(\mathcal{O}_{S}\left(2 K_{S}\right)\right)$ is an irreducible six-dimensional representation of the group $G \cong \mathrm{PSL}_{2}\left(\mathbb{F}_{7}\right)$ by Lemma B.4.

Lemma 5.22. The equality $d=9$ is impossible.

Proof. Suppose that $d=9$. Then $g \leqslant 12$ by Theorem 2.14 and $g \in\{3,8,10\}$ by Lemma 5.17. By Lemma [5.18, we have $S \subset F_{4}$. Arguing as in the proof of Lemma 5.17, we see that $S \subset F_{4} \cap F_{6} \cap F_{8}^{\prime}$ if $g=8$. Similarly, we see that $S \subset F_{4} \cap F_{6} \cap F_{14}$ if $g=3$. Thus $g=10$ by Lemma 3.6. Then $S$ is a complete intersection of two cubic surfaces in $\mathbb{P}^{3}$ (see [17, Example 6.4.3]), which is impossible, because there are no $G$-invariant pencils of cubic surfaces by Remark 3.4.

Thus, the cases $(\mathbf{D}),(\mathbf{E}),(\mathbf{F}),(\mathbf{G})$ are not possible.

Lemma 5.23. The equality $d=10$ is impossible.

Proof. Suppose that $d=10$. Then $p+q \notin\{2,4,11\}$ by Lemma 5.18 and Remark 5.16. But $g=6+q+p \leqslant 2 d=20$, which implies that $g \in\{8,10,15,17\}$ by Lemma 5.17. Thus, we see that $g=15$ and $p+q=9$. By Lemma 5.18, we see that $S \subset F_{4}$. Arguing as in the proof of Lemma 5.17, we see that the curve $S$ is contained in the intersection $F_{4} \cap F_{6} \cap F_{8}^{\prime}$, which is impossible by Lemma 3.6.

Lemma 5.24. The equality $d=11$ is impossible.

Proof. Suppose that $d=11$. Then $p+q \notin\{0,5,12\}$ by Lemma 5.18 and Remark 5.16. But $g=10+q+p \leqslant 2 d=22$, which implies that $g \in\{10,15,17,22\}$ by Lemma 5.17. Thus, we see that $g=17$ and $p+q=7$. By Lemma 5.18, we see that $S \subset F_{4}$. Arguing as in the proof of Lemma 5.17, we see that the curve $S$ is contained in the intersection $F_{4} \cap F_{6} \cap F_{8}^{\prime}$, which is impossible by Lemma 3.6.

Lemma 5.25. The equality $d=12$ is impossible.

Proof. Suppose that $d=12$. Then $p+q \notin\{3,5,10\}$ by Remark [5.16. But $g=14+q+p \leqslant 2 d=24$, which implies that $g \in\{15,17,22,24\}$ by Lemma 5.17 . Thus either $g=15$ or $g=22$. Arguing as in the proof of Lemma 5.17 and using Lemma 2.16, we see that $S \subset F_{4} \cap F_{6} \cap F_{8}^{\prime}$, which is impossible by Lemma 3.6.

Lemma 5.26. The equality $d=13$ is impossible. 
Proof. Suppose that $d=13$. Then $p+q \notin\{4,6\}$ by Lemma 5.18 and Remark [5.16. But $g=18+q+p \leqslant 2 d=26$, which implies that $g \in\{22,24\}$ by Lemma 5.17. Thus $p+q \in\{4,6\}$, which is a contradiction.

Lemma 5.27. The equality $d=14$ is impossible.

Proof. Suppose that $d=14$. Then $p+q \neq 0$ by Lemma 5.18 and Remark 5.16. But $g=22+q+p \leqslant 2 d=28$, which implies that $g=22$ by Lemma 5.17. Thus $p=q=0$, which is a contradiction.

By Lemmas 5.19, 5.20, 5.21, [5.22, 5.23, 5.24, 5.25, 5.26, 5.27 and Theorem 2.14, one has $d \geqslant 15$, which implies that $d=15$ by Lemma 5.3. Then $p+q \neq 3$ by Lemma 5.18 and Remark 5.16. But $g=26+q+p \leqslant 2 d=30$, which implies that $g=29$ by Lemma 5.17. Thus, we have $p+q=3$, which is a contradiction.

The assertion of Theorem 1.9 is proved.

\section{Proof of Theorem 1.10}

Throughout this section we use assumptions and notation of Theorem 1.10, and we identify the threefold $X$ with its anticanonical image in $\mathbb{P}^{13}$ (cf. Theorem 4.5). Suppose that Theorem 1.10 is false. Let us derive a contradiction.

Lemma 6.1. There is a $G$-invariant linear system $\mathcal{M}$ without fixed components on $X$ such that $\mathbb{N} \mathbb{C S}(X, \lambda \mathcal{M}) \neq \varnothing$, where $\lambda$ is a positive rational number such that $-K_{X} \sim_{\mathbb{Q}} \lambda \mathcal{M}$.

Proof. The required assertion is well-known (see [10], [5, Corollary A.18]).

Lemma 6.2. Let $\Lambda$ be a union of all curves in $\mathbb{N L} \mathbb{C S}(X, 2 \lambda \mathcal{M})$. Then $\operatorname{deg}(\Lambda) \leqslant 21$.

Proof. Let $M_{1}$ and $M_{2}$ be general surfaces in $\mathcal{M}$, and let $H$ be a general surface in the linear system $\left|-K_{X}\right|$. By Theorem 2.5, we have

$$
22 / \lambda^{2}=H \cdot M_{1} \cdot M_{2} \geqslant \sum_{P \in \Lambda \cap H} \operatorname{mult}_{P}\left(M_{1} \cdot M_{2}\right)>(H \cdot \Lambda) / \lambda^{2}=\operatorname{deg}(\Lambda) / \lambda^{2},
$$

which implies that $\operatorname{deg}(\Lambda) \leqslant 21$. Put

By Lemma 2.8, the set $\mathbb{N L} \mathbb{C S}(X, 2 \lambda \mathcal{M})$ contains every center in $\mathbb{N} \mathbb{C} S(X, \lambda \mathcal{M})$.

$$
\hat{\mu}=\sup \{\epsilon \in \mathbb{Q} \mid \text { the log pair }(X, \epsilon \mathcal{M}) \text { is } \log \text { canonical }\}<2 \lambda,
$$

let $\Sigma_{8}$ be the unique $G$-invariant subset of the threefold $X$ consisting of 8 points (see Lemma 6.3), and let $F$ be the unique $G$-invariant surface in the linear system $\left|-K_{X}\right|$ (see Corollary 4.6).

Lemma 6.3. Suppose that $\Sigma_{8} \subset \mathbb{L} \mathbb{C S}(X, \hat{\mu} \mathcal{M})$. Then $\Sigma_{8} \not \subset \mathbb{N} \mathbb{C}(X, \lambda \mathcal{M})$, and the set $\mathbb{N L} \mathbb{C} \mathbb{S}(X, 2 \lambda \mathcal{M})$ does not contain curves that pass through a point in $\Sigma_{8}$.

Proof. Take $\hat{\epsilon} \in \mathbb{Q}$ such that $\hat{\mu}<\hat{\epsilon} \hat{\mu}<2 \lambda$. By Lemma 2.13, there is a $G$-invariant linear system without fixed components $\hat{\mathcal{B}}$ on $X$, and there are $\hat{\epsilon}_{1} \in \mathbb{Q} \ni \hat{\epsilon}_{2}$ such that $1 \geqslant \hat{\epsilon}_{1} \gg \hat{\epsilon}_{2} \geqslant 0$ and $\mathbb{L} \mathbb{C S}\left(X, \hat{\epsilon}_{1} \hat{\mu} \mathcal{M}+\hat{\epsilon}_{2} \hat{\mathcal{B}}\right)=\Sigma_{8}$, the log pair $\left(X, \hat{\epsilon}_{1} \hat{\mu} \mathcal{M}+\hat{\epsilon}_{2} \mathcal{B}\right)$ is $\log$ canonical, and $\hat{\epsilon}_{1} \hat{\mu} \mathcal{M}+\hat{\epsilon}_{2} \mathcal{B} \sim_{\mathbb{Q}} \hat{\epsilon} \hat{\mu} \mathcal{M}$. Put $\hat{D}=\hat{\epsilon}_{1} \hat{\mu} \mathcal{M}+\hat{\epsilon}_{2} \mathcal{B}$.

Let $\mathcal{I}(X, \hat{D})$ is the multiplier ideal sheaf of the $\log$ pair $(X, \hat{D})$, and let $H$ be a general surface in the linear system $\left|-K_{X}\right|$. The sequence of groups

$$
0 \rightarrow H^{0}\left(\mathcal{O}_{X}(H) \otimes \mathcal{I}(X, \hat{D})\right) \rightarrow H^{0}\left(\mathcal{O}_{X}(H)\right) \rightarrow H^{0}\left(\mathcal{O}_{\Sigma_{8}}\right) \rightarrow 0
$$


is exact by Theorem 2.2 , Then $\Sigma_{8}$ imposes independent linear conditions on surfaces in $\left|-K_{X}\right|$.

Let $\mathcal{D}$ be a linear subsystem of the linear system $\left|-K_{X}\right|$ that consists of all surfaces passing through $\Sigma_{8}$. Then it follows from Theorem 4.5 that $\mathcal{D}$ is the unique fivedimensional $G$-invariant linear subsystem of the linear system $\left|-K_{X}\right|$ and $F \notin \mathcal{D}$. It is clear that the base locus of the linear system $\mathcal{D}$ does not contain surfaces.

Suppose that the base locus of the linear system $\mathcal{D}$ does not contain any curve that passes through a point in $\Sigma_{8}$. Let us show that this assumption implies everything we have to prove.

Let $M_{1}$ and $M_{2}$ be general surfaces in the linear system $\mathcal{M}$. Put $M_{1} \cdot M_{1}=\Xi+\Lambda$, where $\Xi$ and $\Lambda$ are effective one-cycles such that $\Sigma_{8} \subset \operatorname{Supp}(\Xi), \Sigma_{8} \not \subset \operatorname{Supp}(\Lambda)$ and $\operatorname{Supp}(\Xi) \cap \operatorname{Supp}(\Lambda)$ consists of at most finitely many points. Let $D$ be a general surface in the linear system $\mathcal{D}$. Since $|\operatorname{Supp}(\Xi) \cap D|<+\infty$, one has

$$
22 / \lambda^{2} \geqslant 22 / \lambda^{2}-D \cdot \Lambda=D \cdot \Xi \geqslant \sum_{P \in \Sigma_{8}} \operatorname{mult}_{P}(\Xi)=8 \operatorname{mult}_{P}(\Xi)
$$

for every point $P \in \Sigma_{8}$. Then $\mathbb{N} \mathbb{C S}(X, \lambda \mathcal{M})$ contains no points in $\Sigma_{8}$ by Theorem 2.7 .

Let $Z$ be a $G$-orbit of an irreducible curve in $X$ such that $\Sigma_{8} \subset Z$. Then

$$
\operatorname{deg}(Z)=D \cdot Z \geqslant \sum_{P \in \Sigma_{8}} \operatorname{mult}_{P}(D) \operatorname{mult}_{P}(Z) \geqslant \sum_{P \in \Sigma_{8}} \operatorname{mult}_{P}(Z) \geqslant 24
$$

by Lemma 2.11. Hence $\mathbb{N L} \mathbb{C S}(X, 2 \lambda \mathcal{M})$ contains no components of the curve $Z$ by Lemma 6.2, which implies that $\mathbb{N L} \mathbb{C} \mathbb{S}(X, 2 \lambda \mathcal{M})$ does not contain curves that pass through a point in $\Sigma_{8}$.

To complete the proof, we must show that the base locus of the linear system $\mathcal{D}$ contains no curves that contain a point in $\Sigma_{8}$. Suppose that this is not true. Let us derive a contradiction.

The base locus of the linear system $\mathcal{D}$ contains a curve $C$ that is a $G$-orbit of an irreducible curve such that $\Sigma_{8} \subset C$. Then $\operatorname{mult}_{P}(C) \geqslant 3$ for every $P \in \Sigma_{8}$ by Lemma 2.11 .

Let $Q_{1}, Q_{2}, Q_{3}$ and $Q_{4}$ be general points in $X$, and let $\mathcal{H}$ be a linear subsystem in $\left|-K_{X}\right|$ that consists of all surfaces that contain the set $\left\{Q_{1}, Q_{2}, Q_{3}, Q_{4}\right\}$. Then $\mathcal{H} \cap \mathcal{D}$ is a pencil, and the base locus of the linear system $\mathcal{H}$ contains no curves.

Let $H$ be a general surface in $\mathcal{H}$, and choose $D_{1}$ and $D_{2}$ to be general surfaces in the pencil $\mathcal{H} \cap \mathcal{D}$. Then

$22=H \cdot D_{2} \cdot D_{2} \geqslant \sum_{i=1}^{4} \operatorname{mult}_{Q_{i}}(H) \operatorname{mult}_{Q_{i}}\left(D_{1}\right) \operatorname{mult}_{Q_{i}}\left(D_{2}\right)+|H \cap C| \geqslant 4+\operatorname{deg}(C)$,

since $\operatorname{deg}(C)=|H \cap C|$. Thus, we see that $\operatorname{deg}(C) \leqslant 18$.

Note that $C \not \subset F$, since $\Sigma_{8} \not \subset F$. Therefore, we have

$$
\sum_{P \in F \cap C} \operatorname{mult}_{P}(C) \leqslant|F \cap C| \leqslant F \cdot C=\operatorname{deg}(C) \leqslant 18,
$$

because $C \not \subset F$. Applying Theorem 4.8, we see that $\operatorname{deg}(C)=F \cdot C=|F \cap C|=14$ and $C$ is smooth at every point of the set $F \cap C$. Then $C$ is reducible by Lemma 2.15,

Put $C=\sum_{i=1}^{r} C_{i}$, where $C_{i}$ is an irreducible curve and $r \in \mathbb{Z}_{>0}$. Then one has $r=14 / \operatorname{deg}\left(C_{1}\right)$. By Corollary B.2, either $\operatorname{deg}\left(C_{1}\right)=1$ or $\operatorname{deg}\left(C_{1}\right)=2$. On the other hand, we know that $\operatorname{deg}\left(C_{1}\right)+1 \geqslant\left|C_{1} \cap \Sigma_{8}\right|$, since $\Sigma_{8}$ imposes independent linear 
conditions on surfaces in $\left|-K_{X}\right|$. Hence, we must have

$$
\frac{14}{\operatorname{deg}\left(C_{1}\right)}=r \geqslant \frac{8 \cdot 3}{\left|C_{1} \cap \Sigma_{8}\right|}
$$

because $\operatorname{mult}_{P}(C) \geqslant 3$ for every point $P \in \Sigma_{8}$. Therefore $\left|C_{1} \cap \Sigma_{8}\right|=1+\operatorname{deg}\left(C_{1}\right)$.

Note that $\operatorname{deg}\left(C_{1}\right) \neq 1$. Indeed, if $\operatorname{deg}\left(C_{1}\right)=1$, then $\left|C_{1} \cap \Sigma_{8}\right|=2$ and $r=28$, because the group $G$ acts doubly transitive on the set $\Sigma_{8}$ (see [15, p. 173]), which is a contradiction.

Thus, we see that $C_{1}$ is an irreducible conic and $r=7$, so that $\left|C_{i} \cap \Sigma_{8}\right|=3$.

Let $\Pi_{1}$ be a plane in $\mathbb{P}^{13}$ such that $C_{1} \subset \Pi_{1}$. Then $\Pi_{1}$ contains 3 lines $L_{1}^{1}$, $L_{1}^{2}$ and $L_{1}^{3}$ such that $\left|L_{1}^{1} \cap \Sigma_{8}\right|=\left|L_{1}^{2} \cap \Sigma_{8}\right|=\left|L_{1}^{3} \cap \Sigma_{8}\right|=2$, and the $G$-orbit of the line $L_{1}^{1}$ consists of 28 different lines, since $G$ acts doubly transitive on $\Sigma_{8}$.

Now one can easily see that the $G$-orbit of the plane $\Pi_{1}$ consists of at least $28 / 3>9$ planes, which is impossible since the $G$-orbit of $\Pi_{1}$ consists of at most $r=7$ planes.

By Lemma 6.3, the set $\mathbb{N L} \mathbb{C S}(X, 2 \lambda \mathcal{M})$ contains a center that is not contained in $\Sigma_{8}$. Put

$\mu=\sup \left\{\epsilon \in \mathbb{Q} \mid\right.$ the log pair $(X, \epsilon \mathcal{M})$ is log canonical outside of the subset $\left.\Sigma_{8}\right\}$

in the case when $\Sigma_{8} \subset \mathbb{L} \mathbb{C S}(X, \hat{\mu} \mathcal{M})$. If $\Sigma_{8} \not \subset \mathbb{L} \mathbb{C S}(X, \hat{\mu} \mathcal{M})$, then put $\mu=\hat{\mu}$.

Lemma 6.4. There exist a minimal center in $\mathbb{L} \mathbb{C S}(X, \mu \mathcal{M})$ that is not a point of the set $\Sigma_{8}$.

Proof. This immediately follows from Lemma 6.3.

Let $S$ be a minimal center in $\mathbb{L} \mathbb{C S}(X, \mu \mathcal{M})$ such that $S \not \subset \Sigma_{8}$, and let $Z$ be its $G$-orbit.

Remark 6.5. If $Z=S$ and $S$ is a curve, then $Z \cap \Sigma_{8}=\varnothing$ by Theorem 2.10 and Lemma 2.15.

Let $\epsilon$ be any rational number such that $\mu<\epsilon \mu<2 \lambda$. Then it follows from Lemma 2.13 that there exists a $G$-invariant linear system $\mathcal{B}$ on $X$ such that $\mathcal{B}$ does not have fixed components, and there exist positive rational numbers $\epsilon_{1}$ and $\epsilon_{2}$ such that $1 \geqslant \epsilon_{1} \gg \epsilon_{2} \geqslant 0$ and

$$
\mathbb{L} \mathbb{C S}\left(X, \epsilon_{1} \mu \mathcal{M}+\epsilon_{2} \mathcal{B}\right)=\left(\bigsqcup_{g \in G}\{g(S)\}\right) \bigsqcup \mathbb{N L} \mathbb{C} \mathbb{S}(X, \mu \mathcal{M}),
$$

the log pair $\left(X, \epsilon_{1} \mu \mathcal{M}+\epsilon_{2} \mathcal{B}\right)$ is $\log$ canonical at every point of the subvariety $Z$, and $\epsilon_{1} \mu \mathcal{M}+\epsilon_{2} \mathcal{B} \sim_{\mathbb{Q}} \epsilon \mu \mathcal{M}$. Put $D=\epsilon_{1} \mu \mathcal{M}+\epsilon_{2} \mathcal{B}$. By Lemma 6.4. we may have the following cases:

(A) $\operatorname{LCS}(X, D)=Z$ and the log pair $(X, D)$ is $\log$ canonical,

(B) $\operatorname{LCS}(X, D)=Z \sqcup \Sigma_{8}$ and $Z$ is a finite set,

(C) $\operatorname{LCS}(X, D)=Z \sqcup \Sigma_{8}$ and $Z$ is a curve.

Let $\mathcal{L}$ be the union of all connected components of the subscheme $\mathcal{L}(X, D)$ whose supports do not contains any component of the subvariety $Z$. Then

$$
\operatorname{Supp}(\mathcal{L})=\left\{\begin{array}{l}
\varnothing \text { in the case }(\mathbf{A}), \\
\Sigma_{8} \text { in the cases }(\mathbf{B}) \text { and }(\mathbf{C}) .
\end{array}\right.
$$


Let $\mathcal{I}(X, D)$ be the multiplier ideal sheaf of the $\log$ pair $(X, D)$, and let $H$ be a general surface in the linear system $\left|-K_{X}\right|$. Then

$$
h^{0}\left(\mathcal{O}_{Z} \otimes \mathcal{O}_{X}(H)\right)=14-h^{0}\left(\mathcal{O}_{X}(H) \otimes \mathcal{I}(X, D)\right)-h^{0}\left(\mathcal{O}_{\mathcal{L}}\right) \leqslant 14
$$

by Theorem 2.2, since $\mathcal{L}$ is at most a zero-dimensional subscheme. We have $h^{0}\left(\mathcal{O}_{\mathcal{L}}\right) \in\{0,8\}$.

Corollary 6.7. If $Z$ is a finite set, then $|Z| \leqslant 14$ and the points of $Z$ impose independent linear conditions on surfaces in $\left|-K_{X}\right|$.

Lemma 6.8. The subvariety $S$ is a curve.

Proof. Suppose that $S$ is not a curve. Then $|Z|=14$ by Theorem 4.8 and Corollary 6.7, because we know that $Z \neq \Sigma_{8}$. Note that $Z$ is not contained in any surface in $\left|-K_{X}\right|$ by Corollary6.7, Let $\mathcal{R}$ be a linear subsystem of the linear system $\left|-2 K_{X}\right|$ that consists of all surfaces in $\left|-2 K_{X}\right|$ that pass through the set $Z$. Then its base locus consists of the set $Z$ by [16, Theorem 2].

Let $M_{1}$ and $M_{2}$ be general surfaces in the linear system $\mathcal{M}$. Put $M_{1} \cdot M_{1}=\Xi+\Lambda$, where $\Xi$ and $\Lambda$ are effective one-cycles such that $Z \subset \operatorname{Supp}(\Xi)$ and $Z \not \subset \operatorname{Supp}(\Lambda)$ and $\operatorname{Supp}(\Xi) \cap \operatorname{Supp}(\Lambda)$ consists of at most finitely many points. If $S \in \mathbb{N} \mathbb{C}(X, \lambda \mathcal{M})$, then $\operatorname{mult}_{S}(\Xi)>4 / \lambda^{2}$ by Theorem 2.7 . Let $R$ be a general surface in the linear system $\mathcal{R}$. Then

$$
44 / \lambda^{2} \geqslant 44 / \lambda^{2}-R \cdot \Lambda=R \cdot \Xi \geqslant \sum_{P \in Z} \operatorname{mult}_{P}(\Xi)=14 \operatorname{mult}_{S}(\Xi),
$$

which implies that $g(S) \notin \mathbb{N} \mathbb{C S}(X, \lambda \mathcal{M})$ for every $g \in G$.

By Lemma 6.3, the set $\mathbb{N} \mathbb{C} \mathbb{S}(X, 2 \lambda \mathcal{M})$ contains a center not contained in $\Sigma_{8} \cup Z$. Put

$\bar{\mu}=\sup \left\{\epsilon \in \mathbb{Q} \mid\right.$ the log pair $(X, \epsilon \mathcal{M})$ is $\log$ canonical outside of the set $\left.\Sigma_{8} \cup Z\right\}$.

Note that $\bar{\mu} \geqslant \mu$ and $S \in \mathbb{L} \mathbb{C S}(X, \bar{\mu} \mathcal{M})$. Moreover, if $\bar{\mu}>\mu$, then $S \in$ $\mathbb{N L} \mathbb{C S}(X, \bar{\mu} \mathcal{M})$, because $S \in \mathbb{L} \mathbb{C S}(X, \mu \mathcal{M})$. It follows from Lemma 2.8 that $\bar{\mu}<2 \lambda$.

Let $\Gamma$ be a center in $\mathbb{L} \mathbb{C S}(X, \bar{\mu} \mathcal{M})$ such that $\Gamma \not \subset \Sigma_{8} \sqcup Z$, and let $\Delta$ be its $G$ orbit. Then $\Delta \cap \Sigma_{8}=\varnothing$ by Lemma 6.3. Note that $\Delta \cap Z=\varnothing$ if $\Gamma$ is a point and $\Gamma \in \mathbb{N} \mathbb{C} \mathbb{S}(X, 2 \lambda \mathcal{M})$.

Suppose that the set $\mathbb{N L} \mathbb{C S}(X, 2 \lambda \mathcal{M})$ does not contain curves in $X$ that have a non-empty intersection with the set $Z$. Let us use this assumption to derive a contradiction.

Let $\bar{\epsilon}$ be a rational number such that $\bar{\mu}<\bar{\epsilon} \bar{\mu}<2 \lambda$. Arguing as in the proof of Lemma 2.13, we obtain a $G$-invariant linear system $\mathcal{B}^{\prime}$ on $X$ such that $\mathcal{B}^{\prime}$ does not have fixed components. Moreover, we can choose positive rational numbers $\bar{\epsilon}_{1}$ and $\bar{\epsilon}_{2}$ such that $1 \geqslant \bar{\epsilon}_{1} \gg \bar{\epsilon}_{2} \geqslant 0$ and

$$
\mathbb{L} \mathbb{C S}\left(X, \bar{\epsilon}_{1} \bar{\mu} \mathcal{M}+\bar{\epsilon}_{2} \mathcal{B}^{\prime}\right)=\left(\bigsqcup_{g \in G}\{g(\Gamma)\}\right) \bigsqcup\left(\bigsqcup_{g \in G}\{g(S)\}\right) \bigsqcup \mathbb{N} \mathbb{C} \mathbb{C}(X, \bar{\mu} \mathcal{M})
$$

if $\bar{\mu}=\mu$, or

$$
\mathbb{L} \mathbb{C S}\left(X, \bar{\epsilon}_{1} \bar{\mu} \mathcal{M}+\bar{\epsilon}_{2} \mathcal{B}^{\prime}\right)=\left(\bigsqcup_{g \in G}\{g(\Gamma)\}\right) \bigsqcup \mathbb{N L} \mathbb{C S}(X, \bar{\mu} \mathcal{M})
$$

if $\bar{\mu}>\mu$. 
Put $\bar{D}=\bar{\epsilon}_{1} \bar{\mu} \mathcal{M}+\bar{\epsilon}_{2} \mathcal{B}^{\prime}$. Then $\Gamma$ is a connected component of the subscheme $\mathcal{L}(X, \bar{D})$. Let $\overline{\mathcal{L}}$ be the union of all connected components of the subscheme $\mathcal{L}(X, \bar{D})$ whose supports do not contains any component of the subvariety $\Delta$. Then $Z \subseteq \operatorname{Supp}(\overline{\mathcal{L}})$, which implies that $h^{0}\left(\mathcal{O}_{\overline{\mathcal{L}}} \otimes \mathcal{O}_{X}(H)\right) \geqslant 14$, because $\mathbb{N L} \mathbb{C S}(X, 2 \lambda \mathcal{M})$ does not contain curves that have a non-empty intersection with $Z$.

Let $\mathcal{I}(X, \bar{D})$ be the multiplier ideal sheaf of the log pair $(X, \bar{D})$. Then

$$
0 \leqslant h^{0}\left(\mathcal{O}_{\Delta} \otimes \mathcal{O}_{X}(H)\right)=14-h^{0}\left(\mathcal{O}_{X}(H) \otimes \mathcal{I}(X, \bar{D})\right)-h^{0}\left(\mathcal{O}_{\overline{\mathcal{L}}} \otimes \mathcal{O}_{X}(H)\right) \leqslant 0,
$$

by Theorem 2.2. Thus, we have $h^{0}\left(\mathcal{O}_{\Delta} \otimes \mathcal{O}_{X}(H)\right)=0$, which implies that $\Gamma$ is not a point. It follows from Theorem 2.10 that $\Gamma$ is a smooth curve of genus $g$ such that $H \cdot \Gamma \geqslant 2 g-1$. By Remark 2.12, the curve $\Delta$ is a disjoint union of smooth curves isomorphic to $\Gamma$. Then

$$
0=h^{0}\left(\mathcal{O}_{\Delta} \otimes \mathcal{O}_{X}(H)\right) \geqslant H \cdot \Gamma-g+1>0,
$$

which is a contradiction.

Thus, there is a curve $C_{1} \in \mathbb{N L} \mathbb{C S}(X, 2 \lambda \mathcal{M})$ such that $C_{1} \cap Z \neq \varnothing$. Let $C$ be the $G$-orbit of the curve $C_{1}$. Then $\mathbb{N L} \mathbb{C S}(X, 2 \lambda \mathcal{M})$ contains every irreducible component of the curve $C$, which implies that $\operatorname{deg}(C) \leqslant 21$. Hence, we have $Z \subset C$. But mult $_{P}(C) \geqslant 3$ for every $P \in \Sigma_{8}$ by Lemma 2.11. Thus, we have

$$
42 \geqslant 2 \operatorname{deg}(C)=R \cdot C \geqslant \sum_{P \in Z} \operatorname{mult}_{P}(R) \operatorname{mult}_{P}(C) \geqslant 42,
$$

where $R$ is a general surface in $\mathcal{R}$. Therefore $\operatorname{deg}(C)=21$ and $\operatorname{mult}_{P}(C)=3$ for every $P \in \Sigma_{8}$. Note that $C \not \subset F$, since $Z \not \subset F$. Thus

$$
\operatorname{mult}_{P}(C)|F \cap C| \leqslant \operatorname{mult}_{P}(F \cdot C)|F \cap C|=F \cdot C=\operatorname{deg}(C)=21,
$$

for every point $P \in Z$. So the curve $C$ is smooth at every point of the set $F \cap C$ by Theorem 4.8, which immediately implies that $C$ is reducible by Lemma 2.15.

Put $C=\sum_{i=1}^{r} C_{i}$, where $C_{i}$ is an irreducible curve and $r \in \mathbb{Z}_{>0}$. Then $\operatorname{deg}\left(C_{1}\right) \in\{1,3\}$. But $\operatorname{deg}\left(C_{1}\right)+1 \geqslant\left|C_{1} \cap Z\right|$, because the points of $Z$ impose independent linear conditions on surfaces in $\left|-K_{X}\right|$. We have

$$
\frac{21}{\operatorname{deg}\left(C_{1}\right)}=r \geqslant \frac{14 \cdot 3}{\left|C_{1} \cap Z\right|}
$$

because $\operatorname{mult}_{P}(C) \geqslant 3$ for every point $P \in Z$. Thus $\operatorname{deg}\left(C_{1}\right)=1, r=21$ and $\left|C_{1} \cap Z\right|=2$. Since irreducible components of the curve $C$ are lines and mult $P(C)=3$ for every point $P \in Z$, we can easily see that each connected component of the curve $C$ must have at least 4 components, so that $C$ has at most 6 connected components. Then $C$ is connected by Corollary B.2,

Let $P$ be a point in $Z$, let $G_{P}$ be its stabilizer subgroup in $G$. Then $G_{P} \cong \mathrm{A}_{4}$ by Lemma B.1, and there are exactly 3 irreducible components of the curve $C$ containing $P$, since mult ${ }_{P}(C)=3$. Without loss of generality, we may assume that $P=C_{1} \cap C_{2} \cap C_{3}$. The group $G_{P}$ naturally acts on the set $\left\{C_{1}, C_{2}, C_{3}\right\}$, which implies that there is a subgroup $G_{P}^{\prime} \subset G_{P} \cong \mathrm{A}_{4}$ such that $G_{P}^{\prime}$ acts trivially on $\left\{C_{1}, C_{2}, C_{3}\right\}$ and $G_{P}^{\prime} \cong \mathbb{Z}_{2} \times \mathbb{Z}_{2}$. Note that $C_{1}$ is $G_{P}^{\prime}$-invariant. Let $\bar{P}$ be the point in $Z \cap C_{1}$ such that $\bar{P} \neq P$, let $G_{\bar{P}}$ be its stabilizer subgroup in $G$. Then

$$
\mathbb{Z}_{2} \times \mathbb{Z}_{2} \cong G_{P}^{\prime} \subset G_{\bar{P}} \cong \mathrm{A}_{4},
$$

because $Z \cap C_{1}=\{P, \bar{P}\}$. But the group $G_{\bar{P}}$ contains a unique subgroup that is isomorphic to $G_{P}^{\prime}$, which very easily (almost immediately) implies that every point 
of the set $Z$ is $G_{P}^{\prime}$-invariant, which is impossible, since the group $G$ acts faithfully on $Z$, because the group $G$ is simple.

By Theorem 2.10, the curve $S$ is a smooth curve of genus $g$ such that $\operatorname{deg}(S) \geqslant 2 g-1$. By Remark 2.12, the curve $Z$ is a disjoint union of smooth curves isomorphic to $S$. Let $r$ be the number of connected components of the curve $Z$. Put $d=\operatorname{deg}(S)$. Then

$$
r(d-g+1)=14-h^{0}\left(\mathcal{O}_{X}(H) \otimes \mathcal{I}(X, D)\right)-h^{0}\left(\mathcal{O}_{\mathcal{L}}\right)
$$

by (6.6). Note that $h^{0}\left(\mathcal{O}_{\mathcal{L}}\right)=0$ if and only if $\mathcal{L}(X, D)=Z$. Finally, put $q=h^{0}\left(\mathcal{O}_{X}(H) \otimes \mathcal{I}(X, D)\right)$.

Lemma 6.10. One has $\mathcal{L}(X, D)=Z$.

Proof. Suppose that $Z \subsetneq \mathcal{L}(X, D)$. Then $h^{0}\left(\mathcal{O}_{\mathcal{L}}\right)=8$. It follows from (6.9) that $r(d-g+1)=6-q \leqslant 6$, since $h^{0}\left(\mathcal{O}_{\mathcal{L}}\right) \neq 0$. But $d-g+1 \geqslant 1$, which implies that $r=1$. Therefore $g \leqslant d-g+1=6-q \leqslant 6$, which implies that $g=3$ by Lemma 2.16. If $S \not \subset F$, then $|F \cap S| \leqslant 21$ by Lemma 6.2, which contradicts Lemma 2.15. We see that $S \subset F$. But $q \leqslant 6$, so that $q=1$ by Lemmas 4.5. Thus $d=7$. There is a natural faithful action of the group $G$ on the curve $S$ such that every $G$-invariant divisor on $S$ has even degree by Theorem 2.19. Hence $d$ is even, which is a contradiction.

In particular, we see that the cases $(\mathbf{B})$ and $(\mathbf{C})$ are impossible.

Lemma 6.11. Suppose that $\operatorname{deg}(S)=1$. Then $r \neq 7$.

Proof. Suppose that $r=7$. Then $q=0$ by (6.9). In particular, we have $S \not \subset F$. Then $|F \cap Z| \leqslant F \cdot Z=7$, which contradicts Theorem 4.8, because $F \cap Z$ is $G$-invariant.

Lemma 6.12. The equality $r=1$ holds.

Proof. Suppose that $r \geqslant 2$. Then $r \geqslant 7$ by Corollary B.2. If $r \geqslant 8$, then $d-g+1=1$ by (6.9), which implies that $g=d \geqslant 2 g-1$, which leads to a contradiction.

We see that $r=7$, so that $1 \leqslant d-g+1 \leqslant 2$ by (6.9). Hence $d-g+1=2$, since the equality $d-g+1=1$ leads to a contradiction. Therefore, we have $g=d-1 \geqslant 2 g-2$, which gives $g \leqslant 2$ and $d \leqslant 3$. Therefore $g=0$ and $d=1$, which is impossible by Lemma 6.11.

Thus, there is a natural faithful action of the group $G$ on the curve $S$. We have $d=13+g-q \geqslant 2 g-1$, which implies that $q \leqslant 14-g$. Note that $g \leqslant 14-q \leqslant 14$. Then $g \in\{3,8,10\}$ by Lemma 2.16 .

Lemma 6.13. The curve $S$ is contained in the surface $F$.

Proof. If $S \not \subset F$, then $|F \cap S| \leqslant 21$ by Lemma 6.2, which is impossible by Lemma 2.15.

Applying Lemmas 4.5 and 6.13, we see that $q \in\{1,7,8\}$.

Lemma 6.14. The equality $g=3$ holds.

Proof. Suppose that $g \neq 3$. Then $g \in\{8,10\}$. It follows from (6.9) that $q \leqslant 14-g \leqslant 6$, which implies that $q=1$. Then $d \in\{20,22\}$, which is impossible by Lemmas 2.20 and 2.21 .

Thus, we have $d=16-q$, where $q \in\{1,7,8\}$.

Lemma 6.15. The equality $d=8$ holds. 
Proof. By Theorem 2.19, there is a $G$-invariant line bundle $\theta \in \operatorname{Pic}(S)$ of degree 2 such that $\operatorname{Pic}^{G}(S)$ is generated by $\theta$. In particular, we see that $d$ is even. But $d=16-q$ and $q \in\{1,7,8\}$, so that $d=8$.

Let $Q$ be a surface in the threefold $X$ that is swept out by lines. Then $Q \sim-2 K_{X}$ and the surface $Q$ is irreducible by Lemma 4.7. Note that $Q$ is $G$-invariant.

Lemma 6.16. The curve $S$ is contained in $Q \cap F$.

Proof. By Lemma 6.13, we have $S \subset F$. If $S \not \subset Q$, then $|Q \cap S| \leqslant 2 \operatorname{deg}(S)=16$ which is impossible by Lemma 2.15. Thus, we see that $S \subset Q \cap F$.

The surface $F$ is a smooth $K 3$ surface by Lemma 4.10. Then $S \cdot S=0$ on the surface $X$. Put $\left.Q\right|_{F}=m S+\Delta$, where $m \in \mathbb{Z}_{>0}$, and $\Delta$ is curve such that $S \not \subset \operatorname{Supp}(\Delta)$. Then

$$
16=2 \operatorname{deg}(S)=(m S+\Delta) \cdot S=m S \cdot S+\Delta \cdot S=4 m+\Delta \cdot S,
$$

which implies that $|\Delta \cap S| \leqslant 12$. Hence, it follows from Lemma 2.15 that $\operatorname{Supp}(\Delta) \cap S=\varnothing$, since $\Delta$ is $G$-invariant. Then $\Delta=\varnothing$, because $\operatorname{Supp}(\Delta) \cup S$ is connected (see [17, Corollary 7.9]). Now it follows from (6.17) that $m=4$, which immediately leads to a contradiction, since $44=Q \cdot F \cdot H=4 H \cdot S=32$, where $H$ is a general surface in $\left|-K_{X}\right|$. The obtained contradiction completes the proof of Theorem 1.10.

Proof of Theorem 1.12. Suppose that the pair $(X, R)$ is not log canonical. Put

$$
\mu=\sup \{\epsilon \in \mathbb{Q} \mid \text { the log pair }(X, \epsilon R) \text { is } \log \text { canonical }\}<1 .
$$

Let $S$ be a minimal center of log canonical singularities of the log pair $(X, \mu R)$ (see [21], [7]), and let $Z$ be the $G$-orbit of the subvariety $S$. Then $\operatorname{dim}(S) \leqslant 1$ since $-K_{X}$ generates the group $\operatorname{Pic}(X)$.

Take $\epsilon \in \mathbb{Q}$ such that $1>\epsilon \gg 0$. By Lemma 2.13, there is a $G$-invariant $\mathbb{Q}$-divisor $D$ such that $D \sim_{\mathbb{Q}} \epsilon R$, the singularities of the log pair $(X, D)$ are log canonical, and every minimal center of log canonical singularities of the log pair $(X, D)$ is an irreducible component of the subvariety $Z$.

Let $\mathcal{I}(X, D)$ be the multiplier ideal sheaf of the log pair $(X, D)$. Then the sequence

$$
0 \rightarrow H^{0}\left(\mathcal{O}_{X} \otimes \mathcal{I}(X, D)\right) \rightarrow H^{0}\left(\mathcal{O}_{X}\right) \rightarrow H^{0}\left(\mathcal{O}_{Z}\right) \rightarrow 0
$$

is exact by Theorem 2.2. In particular, we see that $Z$ is connected. By Lemma 2.9. the subvariety $Z$ is irreducible. Hence, we must have $Z=S$.

The variety $X$ does not contain $G$-invariant points by Theorem 4.8 . Thus, we see that $S$ must be a curve. Then $S$ is a smooth and rational curve Theorem 2.10 , which is impossible, because the group $G$ cannot act non-trivially on $\mathbb{P}^{1}$.

\section{Appendix A. Prime Fano threefolds of Degree 22}

In this section we describe Mukai's constructions of prime Fano threefolds of degree 22. Let $F(x, y, z)$ be a quartic form, let $C$ be a curve in $\mathbb{P}^{2}$ defined by

$$
F(x, y, z)=0 \subset \mathbb{P}^{2} \cong \operatorname{Proj}(\mathbb{C}[x, y, z]),
$$

and let $L_{1}, L_{2}, L_{3}, L_{4}, L_{5}, L_{6}$ be six different lines in $\mathbb{P}^{2}$.

Definition A.1. We say that $\sum_{i=1}^{6} L_{i}$ is a hexagon in $\mathbb{P}^{2}$. 
Let $l_{i}(x, y, z)$ is a linear form such that the line $L_{i}$ is given by $l_{i}(x, y, z)=0$.

Definition A.2 ([13, Definition 4.1]). The hexagon $\sum_{i=1}^{6} L_{i}$ is polar to the curve $C$ if $F(x, y, z)=\sum_{i=1}^{6} l_{i}^{4}(x, y, z)$.

Consider $\sum_{i=1}^{6} L_{i}$ as an element of the Hilbert scheme of points in the dual plane $\check{\mathbb{P}}^{2}$.

Definition A.3 ([30, 31], 37]). The variety of polar hexagons to the curve $C$ is

$$
\operatorname{VSP}(C, 6)=\overline{\left\{\Gamma \in \operatorname{Hilb}_{6}\left(\check{\mathbb{P}}^{2}\right) \mid \Gamma \text { is polar to the curve } C\right\}} \subset \operatorname{Hilb}_{6}\left(\check{\mathbb{P}}^{2}\right) .
$$

Put $X=\operatorname{VSP}(C, 6)$. Note that $X$ is a smooth Fano threefold of anticanonical degree 22 provided that $C$ is general (see [30, Theorem 5], [31, Theorem 11]). Below we will assume that $X$ is smooth.

Put $W=\operatorname{Spec}(\mathbb{C}[x, y, z]) \cong \mathbb{C}^{3}$. Then $F(x, y, z) \in \operatorname{Sym}^{4}\left(W^{\vee}\right)$ and the partial derivatives of the form $F(x, y, z)$ give an embedding $\phi: W \rightarrow \operatorname{Sym}^{3}\left(W^{\vee}\right)$.

Suppose that $C$ is not degenerate (see [13, Definition 2.8]). Then every hexagon $\Gamma \in X$ defines a six-dimensional subspace $W_{\Gamma} \subset \operatorname{Sym}^{3}\left(W^{\vee}\right)$ such that $\phi(W) \subset W_{\Gamma}$. This gives a rational map $X \rightarrow \operatorname{Gr}\left(3, U_{7}\right)$, where $U_{7} \cong \operatorname{Sym}^{3}\left(W^{\vee}\right) / W$. The constructed rational map $X \rightarrow \operatorname{Gr}\left(3, U_{7}\right)$ can be extended to an embedding $X \hookrightarrow \operatorname{Gr}\left(3, U_{7}\right)$. This gives an embedding $X \hookrightarrow \mathbb{P}\left(\Lambda^{3}\left(U_{7}\right)\right)$.

There is a natural sequence of maps

$$
\begin{aligned}
\Lambda^{2}(W) \otimes \operatorname{Sym}^{4}(W) \stackrel{\alpha}{\longrightarrow}(W \otimes W) \otimes\left(\operatorname{Sym}^{2}(W) \otimes \operatorname{Sym}^{2}(W)\right) \stackrel{\beta}{\longrightarrow} \\
\stackrel{\beta}{\longrightarrow} \operatorname{Sym}^{3}(W) \otimes \operatorname{Sym}^{3}(W) \stackrel{\gamma}{\longrightarrow} \Lambda^{2}\left(\operatorname{Sym}^{3}(W)\right),
\end{aligned}
$$

and it follows from [13, Section 2.3] that the quartic form $F(x, y, z)$ defines a natural map $\delta_{F}: \operatorname{Sym}^{2}(W) \rightarrow \operatorname{Sym}^{2}\left(W^{\vee}\right)$. Since the quartic $C$ is not degenerate, the map $\delta_{F}$ is invertible. Therefore, there is a natural choice of a non-zero element $\xi \delta_{F}^{-1} \in \operatorname{Sym}^{4}(W)$ via the natural map

$$
\operatorname{Hom}\left(\operatorname{Sym}^{2}\left(W^{\vee}\right), \operatorname{Sym}^{2}(W)\right) \cong \operatorname{Sym}^{2}(W) \otimes \operatorname{Sym}^{2}(W) \stackrel{\xi}{\longrightarrow} \operatorname{Sym}^{4}(W),
$$

which implies that the composition $\gamma \circ \beta \circ \alpha$ gives a map $\zeta: \Lambda^{2}(W) \rightarrow \Lambda^{2}\left(\operatorname{Sym}^{3}(W)\right)$, where $\Lambda^{2}(W) \cong W^{\vee}$.

Lemma A.4. Let $\omega$ be an element in $\operatorname{im}(\zeta)$ considered as a skew form on $\operatorname{Sym}^{3}\left(W^{\vee}\right)$, and let $\Pi \subset \operatorname{Sym}^{3}\left(W^{\vee}\right)$ be the kernel of the form $\omega$. Then $\operatorname{im}(\phi) \subset \Pi$.

Proof. This is a straightforward computation.

By Lemma A.4, the map $\zeta$ gives us a map $W^{\vee} \rightarrow \Lambda^{2}\left(U_{7}^{\vee}\right)$. Therefore, one has

$$
W^{\vee} \otimes U_{7}^{\vee} \stackrel{\sigma}{\rightarrow} \Lambda^{2}\left(U_{7}^{\vee}\right) \otimes U_{7}^{\vee} \stackrel{v}{\rightarrow} \Lambda^{3}\left(U_{7}^{\vee}\right)
$$

so that $v \circ \sigma$ is a monomorphism. Put $U_{14}=\Lambda^{3}\left(U_{7}\right) /\left(W^{\vee} \otimes U_{7}^{\vee}\right) \cong \mathbb{C}^{14}$ and consider $\operatorname{im}(v \circ \sigma)$ as a 21-dimensional linear system of hyperplanes in $\mathbb{P}\left(\Lambda^{3}\left(U_{7}\right)\right)$ vanishing on the image of the threefold $X$. This gives us a natural embedding $X \hookrightarrow \mathbb{P}\left(U_{14}\right)$.

Theorem A.5 (cf. [30], 31], [37]). The embedding $X \hookrightarrow \mathbb{P}\left(U_{14}\right)$ is the anticanonical embedding. 


\section{Appendix B. Representation theory}

In this section we collect some facts about the groups $\mathrm{PSL}_{2}\left(\mathbb{F}_{7}\right)$ and $\mathrm{SL}_{2}\left(\mathbb{F}_{7}\right)$.

Lemma B.1 ([9]). Let $\Gamma$ be a maximal subgroup in $\operatorname{PSL}_{2}\left(\mathbb{F}_{7}\right)$. Then

- either $\Gamma \cong \mathbb{Z}_{7} \rtimes \mathbb{Z}_{3}$ and $\Gamma$ is unique up to conjugation,

- or $\Gamma \cong \mathrm{S}_{4}$ and $\mathrm{PSL}_{2}\left(\mathbb{F}_{7}\right)$ contains two subgroups isomorphic to $\Gamma$ up to conjugation.

Corollary B.2. If $\mathrm{PSL}_{2}\left(\mathbb{F}_{7}\right)$ acts transitively on a finite set $\Sigma$ such that $|\Sigma| \leqslant 41$, then $|\Sigma| \in\{1,7,8,14,21,24,28\}$.

The group $\mathrm{PSL}_{2}\left(\mathbb{F}_{7}\right)$ has exactly six non-isomorphic irreducible representations (see [9]), which we denote by $I, W_{3}, W_{3}^{\vee}, W_{6}, W_{7}, W_{8}$. The values of their characters are listed in the table:

\begin{tabular}{|c|c|c|c|c|c|c|}
\hline & id & $(2)$ & $(3)$ & $(4)$ & $(7)$ & $\left(7^{\prime}\right)$ \\
\hline$\sharp$ & 1 & 21 & 56 & 42 & 24 & 24 \\
\hline$I$ & 1 & 1 & 1 & 1 & 1 & 1 \\
\hline$W_{3}$ & 3 & -1 & 0 & 1 & $\epsilon$ & $\bar{\epsilon}$ \\
\hline$W_{3}^{\vee}$ & 3 & -1 & 0 & 1 & $\bar{\epsilon}$ & $\epsilon$ \\
\hline$W_{6}$ & 6 & 2 & 0 & 0 & -1 & -1 \\
\hline$W_{7}$ & 7 & -1 & 1 & -1 & 0 & 0 \\
\hline$W_{8}$ & 8 & 0 & -1 & 0 & 1 & 1 \\
\hline
\end{tabular}

We use the following notation. The first row represents the conjugacy classes in $\operatorname{PSL}_{2}\left(\mathbb{F}_{7}\right)$ : the symbol id denotes the identity element, the symbol $(n)$ denotes a class of elements of order $n$, the symbols $(7)$ and $\left(7^{\prime}\right)$ denote two different conjugacy classes of elements of order 7 ; note that if $g \in(7)$, then $g^{2} \in(7)$ and $g^{4} \in(7)$, while $g^{3} \in\left(7^{\prime}\right), g^{5} \in\left(7^{\prime}\right)$ and $g^{6} \in\left(7^{\prime}\right)$. The second row lists the number of elements in each conjugacy class. The next six rows list the values of the characters of irreducible representations. By $\epsilon$ we denote the complex number $-1 / 2+\sqrt{-7} / 2$, and by $\bar{\epsilon}$ its complex conjugate.

Looking at the above table, one easily obtains the following corollaries.

Corollary B.3. Let $\Gamma$ be a subgroup of the group $\operatorname{PSL}_{2}\left(\mathbb{F}_{7}\right)$ such that $\Gamma \cong \mathrm{A}_{4}$. Then $W_{3}$ is an irreducible $\Gamma$-representation, and $W_{6}$ is a sum of two irreducible three-dimensional $\Gamma$-representations.

Corollary B.4. The following isomorphisms of the representations of the group $\mathrm{PSL}_{2}\left(\mathbb{F}_{7}\right)$ hold:

$$
\begin{gathered}
\operatorname{Sym}^{2}\left(W_{3}\right) \cong W_{6} \cong \operatorname{Sym}^{2}\left(W_{3}^{\vee}\right), \Lambda^{2}\left(W_{3}^{\vee}\right) \cong W_{3}, \operatorname{Sym}^{3}\left(W_{3}^{\vee}\right) \cong W_{7} \oplus W_{3} \\
\Lambda^{4}\left(W_{7}\right) \cong \Lambda^{3}\left(W_{7}\right)^{\vee} \cong \Lambda^{3}\left(W_{7}\right), W_{7} \otimes W_{3}^{\vee} \cong W_{6} \oplus W_{7} \oplus W_{8} \\
\Lambda^{3}\left(W_{7}\right) \cong I \oplus W_{6} \oplus W_{6} \oplus W_{7} \oplus W_{7} \oplus W_{8}
\end{gathered}
$$

Let $\hat{G}$ be a subgroup in $\mathrm{SL}_{3}(\mathbb{C})$ that is isomorphic to $\mathrm{PSL}_{2}\left(\mathbb{F}_{7}\right)$, and let $\phi: \mathrm{SL}_{3}(\mathbb{C}) \rightarrow \operatorname{Aut}\left(\mathbb{P}^{2}\right)$ be a natural projection. Put $G=\phi(\hat{G})$. Then $G \cong \hat{G}$.

Lemma B.5 ([43, Section 2.10]). There are no $G$-invariant curves in $\mathbb{P}^{2}$ of degrees 1 , 2,3 , and 5 . There is unique $G$-invariant curve in $\mathbb{P}^{2}$ of degree 4 , which is isomorphic to the quartic curve described in Example 1.8. There is unique $G$-invariant curve in $\mathbb{P}^{2}$ of degree 6 , which is isomorphic to the Hessian curve of the quartic curve described in Example 1.8 . 
Lemma B.6. There are no $G$-invariant subsets in $\mathbb{P}^{2}$ consisting of at most 20 points.

Proof. Restricting $W_{3}$ to subgroups of the group $\operatorname{PSL}_{2}\left(\mathbb{F}_{7}\right)$, we obtain the required assertion.

Let $\Gamma$ be a subgroup in $\mathrm{SL}_{2}\left(\mathbb{F}_{7}\right)$, let $\pi: \mathrm{SL}_{2}\left(\mathbb{F}_{7}\right) \rightarrow \mathrm{PSL}_{2}\left(\mathbb{F}_{7}\right)$ be a natural epimorphism. Then $\Gamma \cong 2 . \pi(\Gamma)$ if $\pi(\Gamma)$ is isomorphic to $\mathrm{PSL}_{2}\left(\mathbb{F}_{7}\right), \mathrm{S}_{4}, \mathrm{~A}_{4}, \mathbb{Z}_{7} \rtimes \mathbb{Z}_{3}, \mathrm{D}_{4}$, or $\mathrm{S}_{3}$.

The following table contains the character values of one four-dimensional and one eight-dimensional irreducible representations of the group $\mathrm{SL}_{2}(7)$ and some information about its subgroups:

\begin{tabular}{|c|c|c|c|c|c|c|c|c|c|c|c|}
\hline & id & - id & $(3)_{3}$ & $(3)_{6}$ & $(7)_{7}$ & $(7)_{7}$ & $(7)_{14}$ & $(7)_{14}$ & $(2)_{4}$ & $(4)_{8}$ & $(4)_{8}$ \\
\hline$G$ & 1 & 1 & 56 & 56 & 24 & 24 & 24 & 24 & 42 & 42 & 42 \\
\hline $2 . \mathrm{S}_{4}$ & 1 & 1 & 8 & 8 & 0 & 0 & 0 & 0 & 18 & 6 & 6 \\
\hline $2 . \mathrm{A}_{4}$ & 1 & 1 & 8 & 8 & 0 & 0 & 0 & 0 & 6 & 0 & 0 \\
\hline $2 .\left(\mathbb{Z}_{7} \rtimes \mathbb{Z}_{3}\right)$ & 1 & 1 & 14 & 14 & 3 & 3 & 3 & 3 & 0 & 0 & 0 \\
\hline $2 . D_{4}$ & 1 & 1 & 0 & 0 & 0 & 0 & 0 & 0 & 10 & 2 & 2 \\
\hline $2 . \mathrm{S}_{3}$ & 1 & 1 & 2 & 2 & 0 & 0 & 0 & 0 & 6 & 0 & 0 \\
\hline \hline$U_{4}$ & 4 & -4 & 1 & -1 & $\bar{\alpha}$ & $\alpha$ & $-\alpha$ & $-\bar{\alpha}$ & 0 & 0 & 0 \\
\hline$U_{8}$ & 8 & -8 & -1 & 1 & 1 & 1 & -1 & -1 & 0 & 0 & 0 \\
\hline
\end{tabular}

We use the following notation. The first row represents the conjugacy classes in $\mathrm{SL}_{2}\left(\mathbb{F}_{7}\right)$ : the symbol id denotes the identity element, the symbol -id denotes the element different from id such that $-\mathrm{id} \in \operatorname{ker}(\pi)$, the symbol $(n)_{k}$ denotes a conjugacy class that consists of elements of order $k$ such that their images in $\operatorname{PSL}_{2}\left(\mathbb{F}_{7}\right)$ have order $n$. The next six rows list the number of elements in the corresponding conjugacy classes in some subgroups of the group $\mathrm{SL}_{2}\left(\mathbb{F}_{7}\right)$. The last two rows list the values of the characters of two irreducible representations. The symbol $\alpha$ denotes the complex number $-\left(\zeta^{3}+\zeta^{5}+\zeta^{6}\right)$, where $\zeta$ is a primitive seventh root of unity, and $\bar{\alpha}$ denotes the complex conjugate of $\alpha$.

Lemma B.7. Suppose that $\Gamma \cong 2$. $\left(\mathbb{Z}_{7} \rtimes \mathbb{Z}_{3}\right)$. Then $U_{4} \cong T \oplus J$ and

$$
U_{8} \cong T \oplus T_{1} \oplus J_{1} \oplus J_{2}
$$

as representations of the group $\Gamma$, where $J, J_{1}$ and $J_{1}$ are pairwise non-isomorphic one-dimensional representations, while $T$ and $T_{1}$ are irreducible three-dimensional representations.

Proof. Let $\chi_{4}$ and $\chi_{8}$ be the characters of the representations $U_{4}$ and $U_{8}$, respectively. Then $\left\langle\chi_{4}, \chi_{4}\right\rangle=2$, which immediately implies that $U_{4} \cong J \oplus T$ for some one-dimensional representations $J$ and some irreducible three-dimensional representation $T$ of the group $\Gamma$, because irreducible representations of the group $\Gamma$ are either one-dimensional or three-dimensional. Similarly, we have $\left\langle\chi_{8}, \chi_{8}\right\rangle=4$, which implies that $U_{8} \cong J_{1} \oplus J_{2} \oplus T_{1} \oplus T_{2}$ for some one-dimensional representations $J_{1} \approx J_{2}$ and some irreducible three-dimensional representations $T_{1} \neq T_{2}$ of the group $\Gamma$.

We may assume that $T_{2} \cong T$, because there exist exactly two three-dimensional representations of the group $\Gamma$ with a non-trivial action of its center. But $\left\langle\chi_{4}, \chi_{8}\right\rangle=1$, which implies that that neither $J_{1}$ nor $J_{2}$ is isomorphic to $J$.

Note that we can consider $\mathrm{PSL}_{2}\left(\mathbb{F}_{7}\right)$-representations as $\mathrm{SL}_{2}\left(\mathbb{F}_{7}\right)$-representations.

Lemma B.8. One has $\operatorname{Sym}^{4}\left(U_{4}\right) \cong I \oplus W_{6} \oplus W_{6} \oplus W_{7} \oplus W_{7} \oplus W_{8}$ as representations of the group $\mathrm{PSL}_{2}\left(\mathbb{F}_{7}\right)$ or the group $\mathrm{SL}_{2}\left(\mathbb{F}_{7}\right)$. 
Proof. This follows from elementary and explicit computations (see also [11, Appen$\operatorname{dix} 1])$.

Lemma B.9. As a representation of the group $\Gamma$, the representation $U_{4}$ splits as a sum of two irreducible two-dimensional subrepresentations if $\Gamma \cong 2 . \mathrm{S}_{4}$, a sum of two irreducible two-dimensional subrepresentations if $\Gamma \cong 2 . \mathrm{A}_{4}$, a sum of two irreducible two-dimensional subrepresentations if $\Gamma \cong 2 . \mathrm{D}_{4}$, a sum of an irreducible twodimensional and two non-isomorphic one-dimensional subrepresentations if $\Gamma \cong 2 . \mathrm{S}_{3}$.

Proof. Let $\chi_{4}$ be the character of the representations $U_{4}$. If $\Gamma \cong 2 . \mathrm{D}_{4}$, then $\left\langle\chi_{4}, \chi_{4}\right\rangle=2$, which easily implies that $U_{4}$ splits as a sum of two irreducible twodimensional subrepresentations, because $2 . \mathrm{D}_{4}$ has no odd-dimensional non-trivial irreducible representations.

Since all irreducible representations of the group 2. $\mathrm{A}_{4}$ with a non-trivial action of its center are two-dimensional, the representation $U_{4}$ splits as a sum of two irreducible two-dimensional subrepresentations of the group $\Gamma$ if $\Gamma \cong 2 . \mathrm{A}_{4}$ or $\Gamma \cong 2 . \mathrm{S}_{4}$.

To complete the proof, we may assume that $\Gamma \cong 2 . \mathrm{S}_{3}$. Then there is an epimorphism $\Gamma \rightarrow \mathbb{Z}_{4}$. Let $U$ be the standard unitary two-dimensional irreducible representation of the group $\Gamma$, let $J$ and $J_{1}$ be one-dimensional representations of the group $\Gamma$ that arise from the faithful non-isomorphic one-dimensional representations of the group $\mathbb{Z}_{4}$. Then $U_{4} \cong U \oplus J \oplus J_{1}$ as can be seen from the following table that lists the character values of these representations:

\begin{tabular}{|c|c|c|c|c|c|}
\hline & id & - id & $(3)_{3}$ & $(3)_{6}$ & $(2)_{4}$ \\
\hline $2 . \mathrm{S}_{3}$ & 1 & 1 & 2 & 2 & 6 \\
\hline \hline$U_{4}$ & 4 & -4 & 1 & -1 & 0 \\
\hline$U$ & 2 & -2 & -1 & 1 & 0 \\
\hline$J$ & 4 & -4 & 1 & -1 & $\sqrt{-1}$ \\
\hline$J_{1}$ & 4 & -4 & 1 & -1 & $-\sqrt{-1}$ \\
\hline
\end{tabular}

where we used notation similar to the ones used in the table above.

\section{REFERENCES}

[1] A. Beauville, Non-rationality of the symmetric sextic Fano threefold arXiv:math/1102.1255 (2011)

[2] W. Bosma, J. Cannon, C. Playoust, The Magma algebra system. I. The user language Journal of Symbolic Computation, 24 (1997), 235-265

[3] H. Blichfeldt, Finite collineation groups University of Chicago Press, Chicago, 1917

[4] I. Cheltsov, Log canonical thresholds of del Pezzo surfaces Geometric and Functional Analysis, 18 (2008), 1118-1144

[5] I. Cheltsov, Two local inequalities arXiv:math/0908.0401 (2009)

[6] I. Cheltsov, C. Shramov, Log canonical thresholds of smooth Fano threefolds Russian Mathematical Surveys 63 (2008), 73-180

[7] I. Cheltsov, C. Shramov, On exceptional quotient singularities Geometry and Topology, 15 (2011), 1843-1882

[8] I. Cheltsov, C. Shramov, Five embeddings of one simple group arXiv:math/0910.1783 (2009)

[9] J. Conway, R. Curtis, S. Norton, R. Parker, R. Wilson, Atlas of finite groups Clarendon Press, Oxford, 1985 
[10] A. Corti, Singularities of linear systems and threefold birational geometry L.M.S. Lecture Note Series 281 (2000), 259-312

[11] I. Dolgachev, Invariant stable bundles over modular curves $X(p)$ Contemporary Mathematics 224 (1999), 65-99

[12] I. Dolgachev, V. Iskovskikh, Finite subgroups of the plane Cremona group Birkhauser Boston, Progress in Mathematics 269 (2009), 443-548

[13] I. Dolgachev, V. Kanev, Polar covariants of plane cubics and quartics Advances in Mathematics 98 (1993), 216-301

[14] S. Donaldson, Kähler geometry on toric manifolds, and some other manifolds with large symmetry Handbook of geometric analysis, Advanced Lectures in Mathematics 1 (2008), 29-75

[15] W. Edge, The Klein group in three dimensions Acta Mathematica 79 (1947), 153-223

[16] D. Eisenbud, J-H. Koh, Remarks on points in a projective space MSRI Publications 15, Springer, New York, 157-172

[17] R. Hartshorne, Algebraic geometry Graduate Texts in Mathematics 52 (1977) Springer-Verlag, New York-Heidelberg

[18] M. Gross, S. Popescu, Calabi-Yau threefolds and moduli of abelian surfaces I Compositio Mathematica 127 (2001), 169-228

[19] V. Iskovskikh, Yu. Prokhorov, Fano varieties Encyclopaedia of Mathematical Sciences 47 (1999) Springer, Berlin

[20] Y. Kawamata, On Fujita's freeness conjecture for 3-folds and 4-folds Mathematische Annalen 308 (1997), 491-505

[21] Y. Kawamata, Subadjunction of log canonical divisors II American Journal of Mathematics 120 (1998), 893-899

[22] J. Kollár, Singularities of pairs Proceedings of Symposia in Pure Mathematics 62 (1997), 221-287

[23] R. Lazarsfeld, Positivity in algebraic geometry II Springer-Verlag, Berlin, 2004

[24] S. Levy (editor), The eightfold way. The beauty of Klein's quartic curve, MSRI Publications 35 (1999), Cambridge University Press

[25] C. Mallows, N. Sloane, On the invariants of a linear group of order 336 Proceedings of the Cambridge Philosophical Society 74 (1973), 435-440

[26] N. Manolache, F.-O. Schreyer, Moduli of $(1,7)$-polarized abelian surfaces via syzygies Mathematische Nachrichten 226 (2001), 177-203

[27] A. Marini, On a family of (1,7)-polarised abelian surfaces Mathematica Scandinavica 95 (2004), 181-225

[28] F. Melliez, K. Ranestad, Degenerations of $(1,7)$-polarized abelian surfaces Mathematica Scandinavica 97 (2005), 161-187

[29] S. Mukai, Finite groups of automorphisms of K3 surfaces and the Mathieu group Inventiones Mathematicae 94 (1988), 183-221

[30] S. Mukai, Biregular classification of Fano 3-folds and Fano manifolds of coindex 3 Proceedings of the National Academy of Sciences of the United States of America 9 (1989), 3000-3002

[31] S. Mukai, Fano 3-folds London Mathematical Society Lecture Note Series 179 (1992), 255-263

[32] K. Oguiso, D.-Q.Zhang, The simple group of order 168 and K3 surfaces Complex geometry, Gottingen (2002), 165-184

[33] Yu.Prokhorov, Blow-ups of canonical singularities Algebra (Moscow, 1998), de Gruyter, Berlin (2000), 301-317 
[34] Yu. Prokhorov, Exotic Fano varieties Moscow University Mathematics Bulletin 45 (1990), 36-38

[35] Yu. Prokhorov, Simple finite subgroups of the Cremona group of rank 3 arXiv:math/0908.0678 (2009)

[36] K. Ranestad, F.-O. Schreyer, Varieties of sums of powers Journal fr die Reine und Angewandte Mathematik 525 (2000), 147-181

[37] F.-O. Schreyer, Geometry and algebra of prime Fano 3-folds of genus 12 Compositio Mathematica 127 (2001), 297-319

[38] J.-P. Serre, A Minkowski-style bound for the order of the finite subgroups of the Cremona group of rank 2 over an arbitrary field Moscow Mathematical Journal 9 (2009), 193-208

[39] T. Springer, Invariant theory Lecture Notes in Mathematics 585 (1977), Springer-Verlag, Berlin-New York

[40] G. Tian, On Kähler-Einstein metrics on certain Kähler manifolds with $\mathrm{c}_{1}(M)>0$ Inventiones Mathematicae 89 (1987), 225-246

[41] G. Tian, Kähler-Einstein metrics with positive scalar curvature Inventiones Mathematicae 130 (1997), 1-37

[42] G. Xiao, Galois covers between K3 surfaces Annales de l'Institut Fourier 46 (1996), 73-88

[43] S. Yau, Y. Yu, Gorenstein quotient singularities in dimension three Memoirs of the American Mathematical Society 505 (1993), Providence

University of Edinburgh, Edinburgh EH9 3JZ, UK, I.Cheltsov@ed.ac.uk

Steklov Institute of Mathematics, Moscow 119991, Russia, shramov@mccme.ru

Laboratory of Algebraic Geometry, GU-HSE, 7 Vavilova street, Moscow 117312 , Russia 